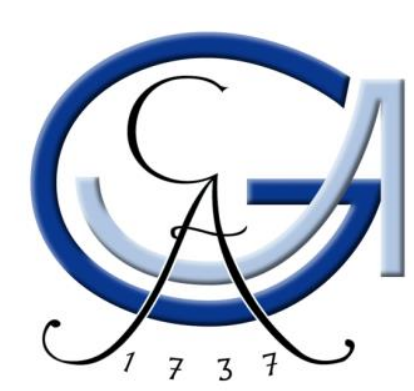

\title{
INCREASING THE ROBUSTNESS OF ACTIVE UPPER LIMB PROSTHESES
}

\author{
Dissertation \\ for the award of the degree \\ "Doctor of Philosophy" Ph.D. Division of Mathematics and Natural Sciences \\ of the Georg-August-Universität Göttingen
}

within the doctoral program PCS

of the Georg-August University School of Science (GAUSS)

Submitted by

Antonietta Stango

From Foggia (Italy)

Goettingen, 2016 


\section{Betreuungsausschuss:}

Prof. Dr. Dario Farina (Referent)

Prof. Dr. Xiaoming Fu (Koreferent)

Weitere Mitglieder der Prüfungskommission:

Prof. Dr. Florentin Wörgötter

Prof. Dr. Stephan Waack

Prof. Dr. Jens Grabowski

Prof. Dr. Luke Russell

Tag der mündlichen Prüfung: 23 November, 2016 


\section{Preface}

This thesis is based on my work done at the Institute for Neurorehabilitation Systems at the University Medical Center Goettingen. My work has been partially founded by German Ministry for Education and Research (BMBF) via the Bernstein Focus Neurotechnology (BFNT) Göttingen under grant number 1GQ0810

The local ethics committee approved all studies involving human subjects, and all subjects signed informed consents prior to their participation in the studies.

The entire thesis has been originally written by me. Part of the materials used in this thesis have also been published in journals or conferences, where I am the first or corresponding author. All rights for re-use of previously published material were obtained. Reused figures and tables of IEEE publications are marked with (C [Year] IEEE.

Hereby I declare that I have written this thesis independently and with no other aids and sources than quoted.

Goettingen, September 6, 2016

Antonietta Stango 


\section{Abstract}

The progresses in mechanics and electronics have provided the technology to build very advanced upper limb prostheses that are multifunctional and capable of several degrees of freedom (DoF). However, these advanced systems are not commonly used by patients. This is due to the lack of intuitive control and to the limited reliability of the man-machine interface. Although research in this field has been very active in the last decades and many control algorithms have been proposed, the control systems offered commercially and in clinics to the patients are basically the same as the original simple schemes dated more than 50 years. In this thesis, the reasons of this discrepancy between technology advance and usage of upper limb prostheses by amputees have been analyzed. Moreover, the robustness and reliability of the myocontrol have been identified as crucial for future developments. Therefore, the thesis contributes in this direction by following two approaches.

In the first part, the non-invasive approach (surface EMG for control) has been addressed. A literature review on control algorithms and in particular pattern recognition has been conducted. These studies revealed that the misplacement of the electrodes caused by the donning and doffing of the prosthesis is one of the major issues limiting the reliability. Furthermore, all previous studies to improve the reliability of pattern recognition algorithms were based on a very small number of EMG electrodes. A novel approach to increase the robustness and reliability of the control algorithm has been developed. The proposed approach reduced the impact of electrodes replacement, and noise on the control performance. The method is based on high-density EMG recordings made with hundreds of closely-spaced electrodes on the forearm muscles. From these recordings, new features based on spatial correlation (variogram) have been proposed. This feature set has been tested on data collected on both able-bodied subjects and one amputee, showing a substantial reduction in the sensitivity to electrode shifts and noise with respect to classic methods. Moreover, the method proposed based on the variogram allowed to eliminate noisy channels during the testing phase without re-training the system. This is one of the 
first studies that explicitly use spatial information from high-density EMG recordings to improve myocontrol, with substantial benefits. The use of such number of electrodes has been always considered a strong drawback for clinical applications. However, the advances in grid technologies and the development of microprocessors capable of handling a high amount of data are supporting the use of HD EMG in myoelectric control. Indeed, new embedded architectures specifically designed for accelerating the signal processing for realtime prosthesis control with HD EMG have been recently presented in the literature.

The second part of the thesis focuses on the invasive approach. With this approach, signals to control the prosthesis are acquired through sensors implanted into the human body. This solution eliminates the problem of the electrodes shift during donning and doffing since the implants are chronically anchored to the muscles. Moreover, invasive EMG signals can be recorded from deep muscles, which are not accessible with surface EMG. This solution can be more challenging than the previous, since it requires surgery, and the transmission of the signals has to be wireless to avoid breakage or infections due to the presence of percutaneous wires. Following a review of the current devices, the major challenges to face the design of an implanted device have been identified. The wireless transmission from implants in the human body to a controller located inside the socket prosthesis has to be carefully studied since the human body is a heterogeneous propagation environment. There are few solutions in the literature and they are characterized by communication links tailored for the application, which use frequency bands not dedicated to implants communication and hence prone to interference. Through accurate simulation studies, a new system has been proposed. The system includes implanted EMG sensors that can record, process and transmit wirelessly EMG signals to an embedded controller positioned inside the socket of the prosthesis. The simulation studies conducted in this part of the thesis provided the channel model measuring the path loss. The safety of the system has been checked simulating the SAR values, and a preliminary link budget analysis confirmed the feasibility of such system. The system proposed has also the advantage that it can be adapted to any kind of amputation, since it doesn't need coils that can limit the positioning of the implants. Moreover, the wireless link follows a standard protocol (IEEE 802.15.6). 
In conclusion, the thesis proposes two directions for improving the robustness in the control of upper limb prostheses, one with advances in non-invasive systems and the other with new concepts in implanted designs. Both directions seem promising and applicable to new generations of upper limb prostheses. While the thesis demonstrates the feasibility of the solutions presented, further developments are needed to translate them into clinical products. 


\section{Acknowledgments}

First I would like to thank Prof. Farina who offered me the opportunity to undertaken this work. He supported me through his guidance and at the same time allowed me to be independent and enterprising. His expertise has been inspiration in the difficulties that unavoidably are encountered during the research work.

Further I would like to thank Prof. Fu without whom I would not be able to enroll in the PhD program. His valuable support in all matter helped me from the initial phase of this work until the end.

I thank Dr. Yazdandoost Kamya from NICT Japan for his collaboration and technical support. He encouraged me to continue my work in the difficult moments and provided me always useful advices.

A great thanks goes also to all my colleagues and friends of the Institute of Neurorehabilitation Systems. With them I had a great time in the Institute.

A special thanks to my family who always supported and encouraged me during these years.

Finally, this thesis would never have started without Francesco. 


\section{Table of Contents}

Preface

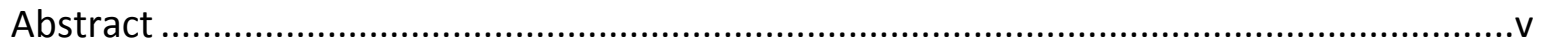

Acknowledgments ...................................................................................................viii

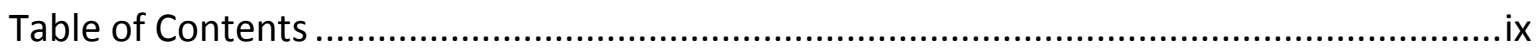

List of abbreviations .................................................................................................

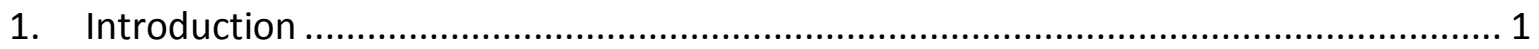

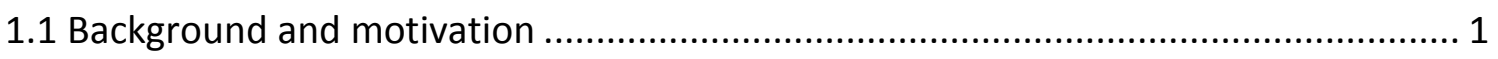

1.2 Limitations and challenges in control of myoelectric hand prostheses...................... 3

1.3 Thesis objective and outline ……...................................................................... 5

Part I: The non-invasive approach ............................................................................... 1

2. State of the art in myocontrol for upper limb prostheses ......................................... 8

2.1 Challenges in the use of surface electrodes in EMG pattern recognition.................. 13

3. Spatial correlation of High Density EMG signals as new robust features for

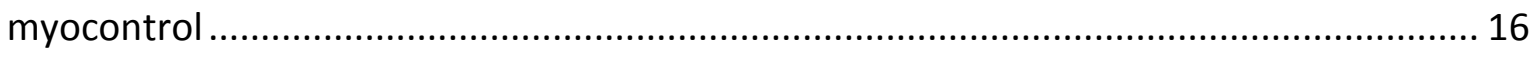

3.1 High Density sEMG in myoelectric control ......................................................... 16

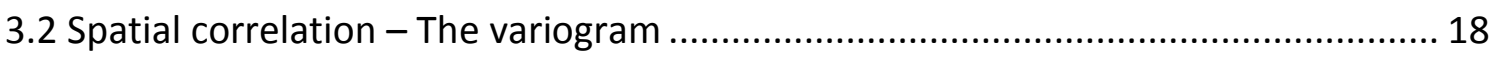

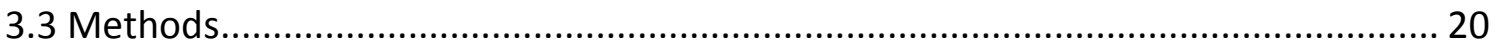

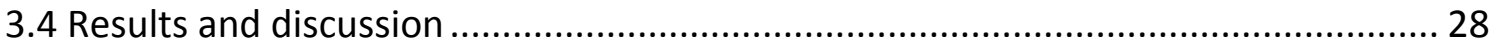

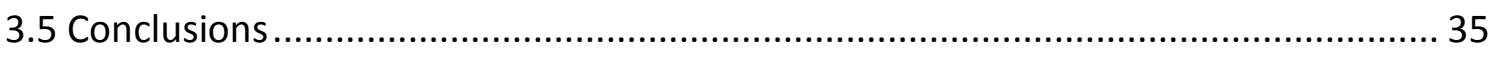

4. Exploitation of spatial features of HD EMG ............................................................ 37

Part II: The invasive approach...................................................................................... 8

5. State of the art in implanted EMG sensors for myocontrol ........................................ 41

6. Background of wireless RF implanted devices....................................................... 45 
6.1 Medical Implant Communication System (MICS) band....................................... 45

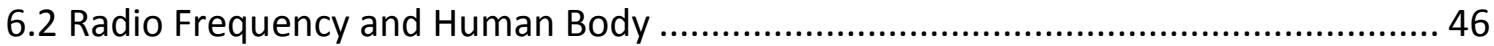

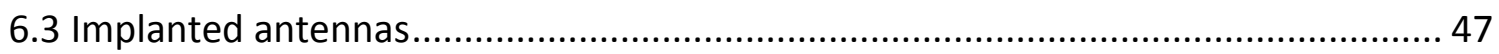

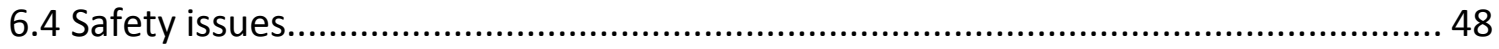

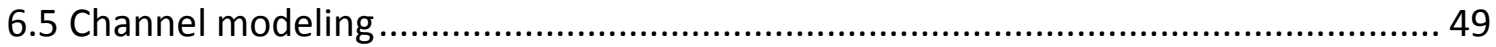

6.6 Finite Difference Time Domain (FDTD) simulations ......................................... 51

6.7 Human model and electromagnetic simulation tool .......................................... 51

7. Characterization of In-Body to On-Body wireless Radio Frequency link for upper limb

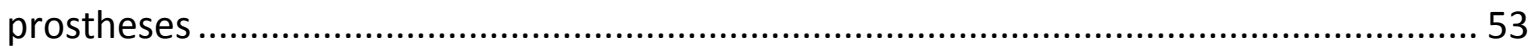

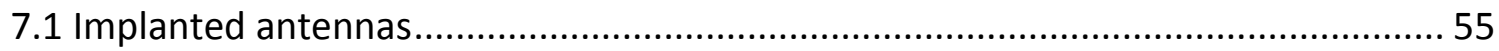

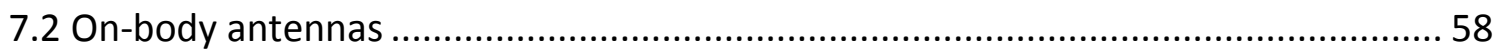

7.3 Channel model for upper limb prostheses ................................................... 61

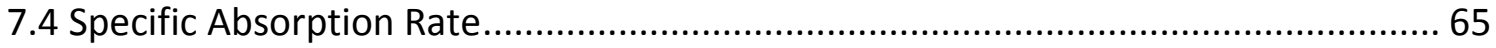

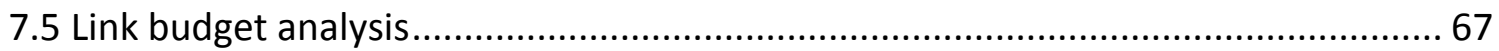

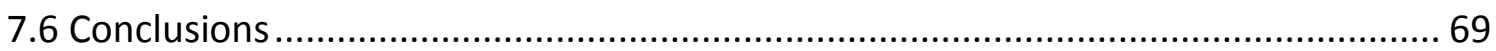

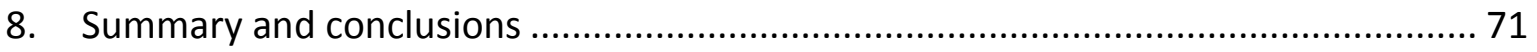

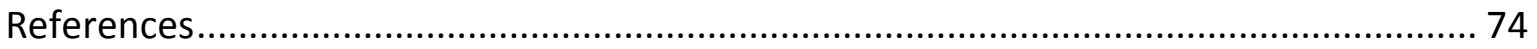

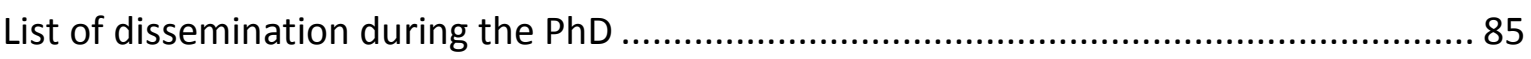

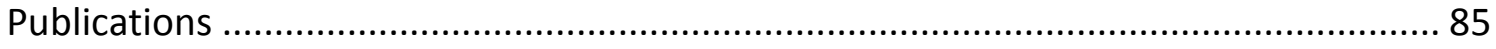

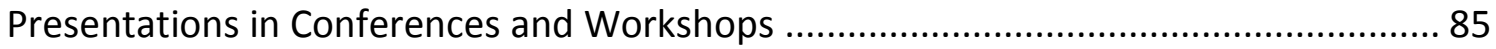

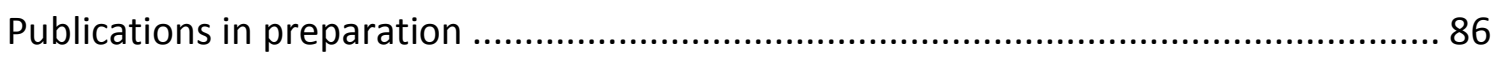

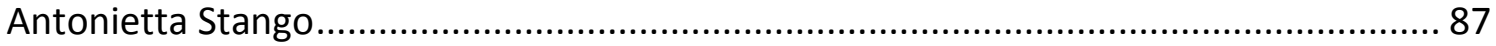




\section{List of abbreviations}

\begin{tabular}{|c|c|}
\hline AR & AutoRegression \\
\hline BW & Bandwidth \\
\hline CSP & Common Spatial Pattern \\
\hline DoF & Degree of Freedom \\
\hline DSIFT & Scale Invariant Feature Transform \\
\hline EEG & Electroencephalogram \\
\hline EIRP & Equivalent Isotropic Radiated Power \\
\hline EMG & Electromyography \\
\hline ETSI & European Telecommunication Standards Institute \\
\hline ETSI & European Telecommunication Standards Institute \\
\hline FCC & Federal Communication Commission \\
\hline FDTD & Finite Difference Time Domain \\
\hline HD & High Density \\
\hline HOW & histogram-of-visual-words \\
\hline ICNIRP & International Commission on Non-lonizing Radiation Protection \\
\hline IEEE & Institute of Electrical and Electronics Engineer \\
\hline ITU & International Telecommunications Union \\
\hline LDA & Linear Discriminant Analysis \\
\hline LM & Link Margin \\
\hline MAC & Media Access Control \\
\hline MICS & Medical Implant Communication System \\
\hline $\mathrm{MRI}$ & Magnetic Resonance Images \\
\hline $\mathrm{NF}$ & Noise Figure \\
\hline OVO & One versus One \\
\hline $\mathrm{PL}$ & Path Loss \\
\hline $\mathrm{RF}$ & Radio frequency \\
\hline RMS & Root Mean Square \\
\hline
\end{tabular}




$\begin{array}{ll}\text { SAR } & \text { Specific Absorption Rate } \\ \text { SEMG } & \text { surface EMG } \\ \text { SIFT } & \text { Dense Scale Invariant Feature Transform } \\ \text { SNR } & \text { Signal to Noise Ratio } \\ \text { SVM } & \text { Support Vector Machine } \\ \text { TD } & \text { Time Domain } \\ \text { TDAR } & \text { Time Domain AutoRegression } \\ \text { TMR } & \text { Target Muscle Reinnervation } \\ \text { Variog } & \text { Variogram } \\ \text { WBAN } & \text { Wireless Body Area Network } \\ \text { WHO } & \text { World Health Organization }\end{array}$




\section{Introduction}

\subsection{Background and motivation}

The loss of an upper limb is a traumatic event, not only for the limited physical functionality that it generates but also because the hands are used for gestures, communications and sensations [1][2]. In most cases, the absence of the hand is a consequence of a trauma or a disease rather than congenital [3] [4] [5]. In case of amputation, the patients have high expectations from the prosthesis and the rehabilitation process. They expect to restore the functionalities of the limb as it was before the amputation. In the last decades, the research in this field has been quite active, indeed several new control systems for multifunction prostheses have been proposed with the purpose to improve the usability and provide functionalities similar to the lost hand.

The upper limb prostheses can be divided in two main categories, passive and active. The passive prostheses are usually only cosmetic. They substitute aesthetically the missing limb and can facilitate some specific activities. The active prostheses instead can be divided in body powered and electric powered. The body powered prostheses are controlled by cables that link the movement of the body to the prosthesis. A certain movement of the body will pull the cables and cause the opening or closing of the terminal device of the prosthesis which can be a hand, a hook or specific tools called prehensors. The last and more recent type of upper limb prostheses are externally powered. The batteries power the motors that can move the components of the prosthesis. This kind of prostheses can be controlled electrically by external buttons or by signals generated by the residual muscles (electromyography, EMG).

The history of prosthetic hands is very long. Already a Roman general in the second Punic War, which had an arm amputated, substituted the limb with an iron hand, as written by Pliny the Elder [6]. Lately there are evidences that artificial hands were inspired by body 


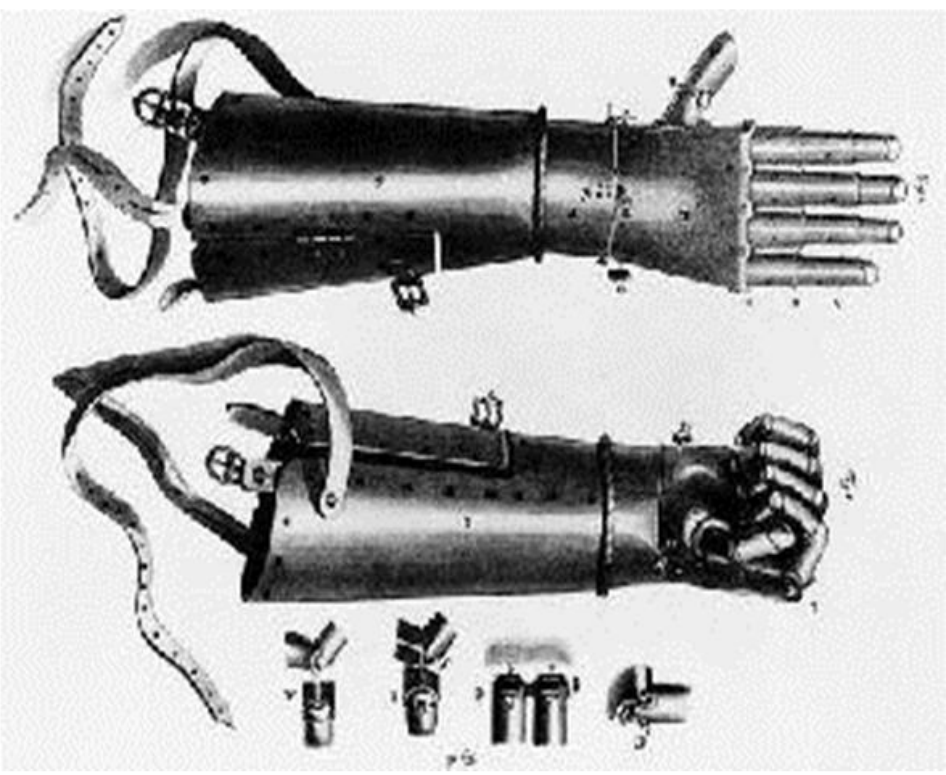

Figure 1. Götz Berlichingen 's prosthetic hand. Source image Wikipedia. The image is in the public domain due to its age

armor and used mainly by knights to replace the limb lost during battles. The most famous example is the prosthetic hand of Götz von Berlichingen, a German knight who lost his hand in a battle in 1504 . The lost hand was substituted by a mechanical prosthesis made of iron (Figure 1) [7]. Most luckily this prosthesis was used only during the battles. Subsequently there have been attempts to improve the functionalities and robustness of the prostheses, introducing joints and rotations of the wrist [7]. In the $19^{\text {th }}$ century many efforts to make the prostheses lighter, using wooden or aluminum have also been done. Only after the World War II, when the numbers of amputees increase considerably, many investments to improve the quality of limb prostheses have been undertaken. Indeed the veterans were dissatisfied from the prostheses provided by the US government and demanded for improved devices [6]. Since then new technologies have been developed and applied in order to obtain devices that can respond better to the needs of the users. The myoelectric prostheses became the most common among the users after the 1960s [8]. In the years after the research in this field has been quite active contributing to improve considerably the prostheses commercially available. Today manufacturers of myoelectric upper limb 
prostheses can claim to have developed multifunction robotic hands controlled trough sensors either placed on the skin of the remaining limb or implanted in muscles, nerves or brain. Nevertheless the prostheses that are commercially available, and used by amputees, are still controlled by few surface EMG electrodes. The control mechanism is still far from being intuitive, moreover any sensory information is provided. Moreover the rate of abandonment of the prostheses is still quite high (>25\%) and can vary across the users [9] [10]. New solutions are needed to better satisfy the users needs and at the same time, they should be easily commercialized. This means that researchers and manufacturers should work to have prostheses that are more functional, according with the requirements of the users, and with a reasonable cost.

Critical technological aspects, that limit the robustness and reliability of the state of the art upper limb prostheses, have been identified. The challenges are related with multiple uses of the prostheses (shift of surface EMG electrodes), the presence of noise while recording and transmitting the EMG signals and finally the reduced number of controllable functions. Therefore, the aim of this thesis is to propose and test two approaches to overcome these difficulties and improve the reliability of myocontrol. The first approach is non-invasive and is based on the use of surface EMG electrodes, while the second is invasive and based on the use of implanted EMG sensors.

\subsection{Limitations and challenges in control of myoelectric hand prostheses}

The control of a device trough signals generating by muscle activity is called myoelectric control. It requires the ability to activate voluntary skeletal muscles by the users. In case of upper limb prosthesis, the EMG signal is associated to a desired movement of the prosthesis.

To record myoelectric signals, surface electrodes, wire or needle electrodes, and implanted electrodes can be used. Surface electrodes can be wet-type or dry-type. The wet, or gel, 
electrodes require conductive gel between the skin and the electrode, and also skin preparation. They provide a good signal quality, but they are not for long term use since the gel can dry out. Instead the dry electrodes are suitable for long term use and, moreover they can be integrated easily in the socket of the prostheses. The dry electrodes are then the only practical solution for the actual commercial available hand prostheses [11]. Finally the wire or needle electrodes are invasive, they have to be inserted into the muscle under the skin. The presence of wires makes them no appropriate for myoelectric prostheses. Implanted electrodes as alternative to dry electrodes in the field of myoelectric prostheses have also been considered, but they are still in the research stage and no solution has been yet commercialized.

In the las few years many progresses in the mechanics of the upper limb prostheses have been carried out. In fact, some of the commercially available prostheses can claim to allow many degrees of freedom (DoFs) which can enable also several type of grasping. There are robotic hands capable of up to 24 grip patterns and with the wrist able to rotate and flex [12]. The research in this field has been very active, many scientific papers have been written proposing new control methods, sensory feed backs solutions and lately also the introduction of implanted EMG electrodes.

Despite the advances that the research and technology have made in the last decades the hand prostheses available in the market are still far from offering intuitive, multifunctioning prostheses and especially they do not provide sensations. The control system in most of the cases still relies on two or three surface dry electrodes and the control strategy is often sequential. It means that a specific signal is used to switch between a set of predefined movements [12]. The discrepancy between advancements in research and technology, and prostheses effectively used by the amputee represents one of the challenges in this field that need to be taken. Very often the solutions presented in literature do not take into account the amputees requirements, the experiments are done in laboratory under certain conditions that do not represent the everyday life[13]. 


\subsection{Thesis objective and outline}

This work intends to analyze some of the problems that are preventing the utilization of more technological upper limb prostheses and proposing new solutions. The thesis analyzes two different approaches in the control of upper limb prostheses. The first part concerns the non-invasive approach and hence the use of surface EMG electrodes. The second part take into account the invasive approach and that is the use of implanted devices to record EMG signals. The final objective of this thesis is not to evaluate which approach is better, but provide two different solutions that can be offered to amputee.

The outline of the thesis is structured as follows:

Part I: The non-invasive approach.

- In Chapter 2 a review on the state of the art of sEMG upper limb prostheses is given. An overview of the controlling algorithms is also provided, with particular attention to the pattern recognition algorithms. The main features used in literature have been summarized. Finally, the issues related to pattern recognition algorithms are carried out, outlying the shift of electrodes as a major problem in the robustness of such algorithm for control of upper limb prostheses.

- In Chapter 3 a new method to provide features robust to electrodes number and shift has been proposed. The method is based on the use of the spatial correlation of high density EMG signals (the variogram), which have not been used previously for controlling upper limb prostheses. The method is explained and tested on data acquired on able- bodied subject and one amputee. The accuracies of classification obtained with this method are compared with those of the most popular algorithms used in pattern recognition for upper limb prostheses. Finally, it has also been tested that the variogram features allow to eliminate channels that may be broken without re-training. 
- In Chapter 4 is given an overview of the studies that have been exploited the spatial correlation and HD EMG after the publication of the method described in the chapter 3. Moreover, the limitations of HD EMG are described.

Part II: The invasive approach.

- In Chapter 5 it is outlined as the problems that are affecting the pattern recognition algorithm in the control of upper limb prostheses can be solved with a different approach, using implanted EMG sensors. A description of the technology available in this field and the state of the art of implanted EMG in robotic hands has been provided. It has been highlighted as the solutions proposed until now in research have not been commercialized. Few solutions have been proposed, and the research in this field is still open.

- In Chapter 6 has been described the background of wireless implanted devices. These devices have specific issues since the medium of propagation is the human body. In this chapter have been described all the aspects that have to be taken into account when designing a device that has to be implanted into the human body and that has to transmit data outside.

- In Chapter 7 it has been proposed a new solution for the wireless transmission of EMG signals recorded inside the human body. The solution proposed is based on a standard communication protocol (IEEE 802.15.6). To define the channel model and prove the safety of the system proposed, two implanted antennas have been implanted in a 3D human model and an electromagnetic simulator which use the FDTD method have been used. The feasibility of the link has been confirmed with a preliminary link budget analysis.

- In Chapter 8 the summary of the thesis is provided. Moreover, the conclusions are discussed, highlighting the pros and drawbacks of the approaches proposed. 
Part I: The non-invasive approach 


\section{State of the art in myocontrol for upper limb}

\section{prostheses}

The developing of current myoelectric upper limb prostheses has been characterized by the use of few surface EMG electrodes. The focus has been on a small number of electrodes because better clinical acceptance and low computational load. In the commercially available upper limb prostheses, the control is usually realized with one or two electrodes [11]. It depends on the condition of the stump. If the stump is small, a single-site system can be used, otherwise two electrodes are positioned over the residual flexor and extensor muscles. To eliminate potential noise, the EMG signals acquired through the surface electrodes are filtered. An estimation of the amplitude or the rate of change of the myoelectric signal provides a measure of the muscles activity level [15]. The contraction of muscle is detected by reaching a threshold that has been determined in the fitting phase of the prostheses. The control scheme that uses such signal processing is called direct control. The direct control can be a simple switch or proportional. In the first case, a contraction of the flexor can close the hand, while a contraction of the extensor can open it. To switch to another DoF a co-contraction, a simultaneous contraction of both muscles, is needed. If the amputees are able to modulate the strength of the muscle contraction, it is possible to control the speed and the force of the prosthetic device (proportional control scheme). The direct control schemes are usually adopted by prosthesis with only one DoF, since the use of co-contraction, to sequentially switch between different DoFs, makes the device slow, and difficult to use [11]. To overcome these difficulties, and allow more DoFs, simultaneous and proportional control, new approaches have been investigated and proposed. In particular, control strategies based on pattern recognition control have been developed. This type of control is based on the concept that amputees can still generate different and repeatable EMG signal patterns associated with each class of movements. The EMG signal 


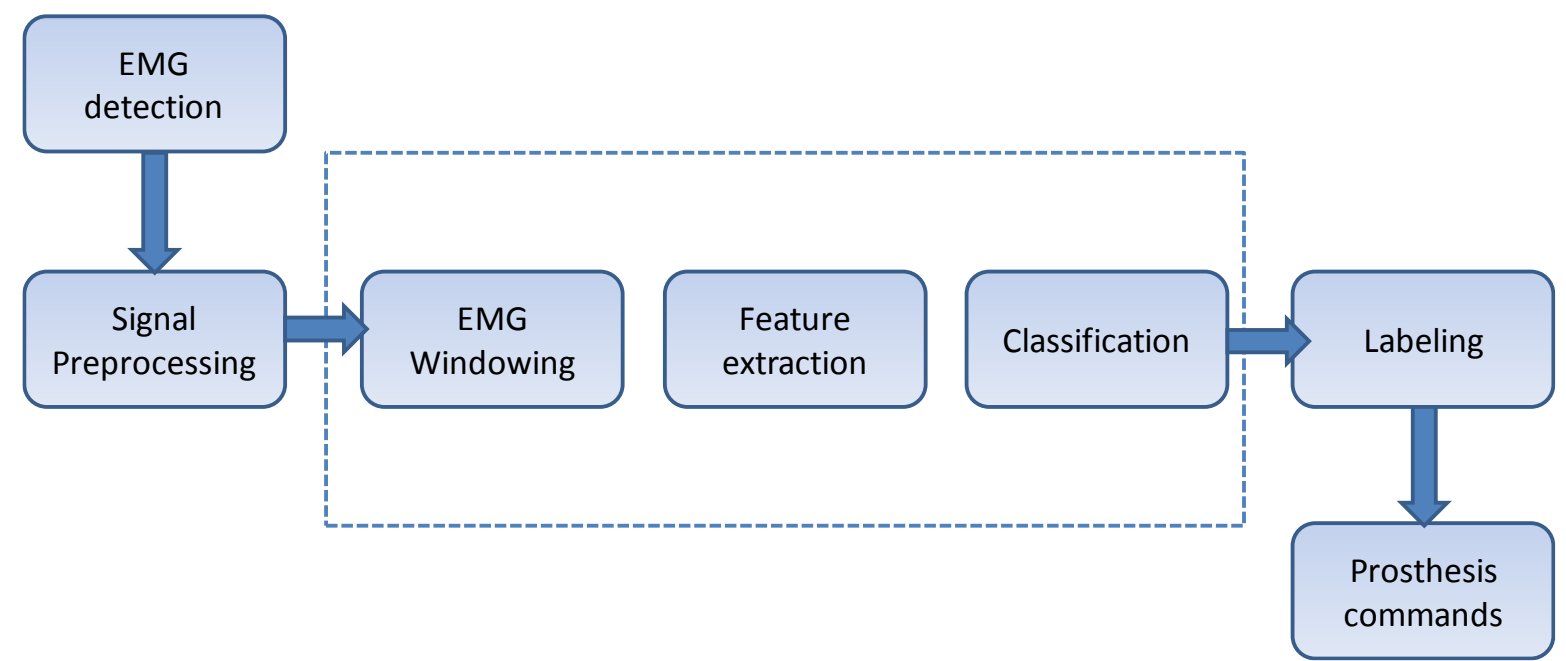

Figure 2. EMG signal processing for pattern recognition algorithms.

processing for pattern recognition algorithms can be summarized by Figure 2 . The first step is the detection of EMG signals from the stump, following by a preprocessing to remove noise and artifact due to electrode movement, at this point start the pattern recognition. It consists of a first stage where the EMG signals are windowed. The next step is the extraction of the features that increase the information of the EMG signals. The last step of the pattern recognition algorithm is the classification of the movements, which provide the class labels to the motor control of the prosthesis.

The selection of the window length is very important to balance between stability of the features and delay in the classification decision [16]. A longer length of the windows reduces the variance of the feature and increases the classification accuracy, but delays the decision [16]. It has been found that the optimal window length is between 150 and $250 \mathrm{~ms}$ [17].

The features extraction is the core part of the pattern recognition control. Several algorithms and features have been investigated with the purpose to identify the most robust EMG pattern [18]. There are algorithms very simple, which consider as only feature the root mean square (RMS) of EMG signals [19]. Others approaches use time domain (TD) 
features [15], [21], [22]. Many TD features have been extracted. The most used are the following [21], where $x_{i}$ is the ith sample of the EMG signal (X), and $\mathrm{N}$ is the window size:

- Mean Absolute Value (MAV). - Estimation of the mean absolute value of the signal

$$
\bar{X}=\frac{1}{N} \sum_{i=1}^{N}\left|x_{i}\right|
$$

- Zero Crossing $(\mathrm{ZC})$. - The number of times the signal passes the zero amplitude axis within an analysis window. A threshold $(\varepsilon)$ must be considered to reduce the noise induced zero crossing. Given two consecutive samples $x_{i}$ and $x_{i+1} Z C$ is incremented if

$$
\begin{gathered}
\left\{x_{k}>0 \text { and } x_{k+1}<0\right\} \text { or }\left\{x_{k}<0 \text { and } x_{k+1}>0\right\} \\
\text { and }\left|x_{k}-x_{k+1}\right| \geq \varepsilon
\end{gathered}
$$

- Slope Sign Changes. - The number of time the slope of the signal changes sign.

Considering a threshold $(\varepsilon)$ to reduce noise induced count. The slopeSign count increased by one if

$$
\begin{gathered}
\left\{x_{i}>x_{i-1} \text { and } x_{i}>x_{i+1}\right\} \text { or }\left\{x_{i}<x_{i-1} \text { and } x_{i}<x_{i+1}\right\} \\
\quad \text { and }\left|x_{i}-x_{i+1}\right| \geq \varepsilon \text { or }\left|x_{i}-x_{i-1}\right| \geq \varepsilon
\end{gathered}
$$

- Waveform Length (WL). - The cumulative length of the EMG signal within the analysis window.

$$
\begin{aligned}
& W L=\sum_{i=1}^{N}\left|\Delta x_{i}\right| \\
& \text { where } \Delta \mathrm{x}_{\mathrm{i}}=\mathrm{x}_{\mathrm{i}}-\mathrm{x}_{\mathrm{i}-1} .6
\end{aligned}
$$

The following have been also considered [22][18] [23]: 
- Mean Absolute Slope (MAS). - The difference between sums in adjacent segments

$$
\Delta \overline{X_{i}}=\overline{X_{i+1}}-\overline{X_{i}}
$$

- Willison amplitude (WAMP). - The amount of times that the change in EMG signal amplitude exceeds a predefined threshold $(\varepsilon)$. It is an indicator of muscle contraction level.

$$
W A M P=\sum_{i=1}^{N} f\left(\left|x_{k}-X_{k+1}\right|\right)
$$

where $f(x)=1$ if $x>\varepsilon, 0$ otherwise.

- Variance (var). - It is a measure of the power of the EMG signal.

$$
\text { var }=\sigma^{2}=\frac{1}{N-1} \sum_{i=1}^{N} x(k)^{2}
$$

- Autoregressive coefficient (AR). - The EMG signal can be considered, in a short interval, as a stationary Gaussian process. The EMG time series can be represented as

$$
x_{i}=\sum_{k=1}^{p} a_{k} x_{i-1}+e_{i}
$$

where $a_{k}$ is the autoregressive coefficient, $p$ is the AR model order, and $e_{i}$ is the residual white noise.

- Cepstrum coefficients (Ceps). - Provide information about the rate of change in different frequency spectrum bands of a signal. The Cepstrum of a signal is defined as the inverse Fourier transform of the logarithm of the magnitude of the power spectrum of the signal data. The Cepstrum coefficients are derived from the autoregressive model and are computed as

$$
c_{1}=-a_{1}
$$




$$
c_{k}=-a_{k}-\sum_{l=1}^{k-1}\left(1-\frac{l}{k}\right) a_{n} c_{k-1}
$$

where $a_{k}$ is the $\mathrm{k}^{\text {th }}$ AR coefficient as described above, $c_{k}$ is $\mathrm{k}^{\text {th }}$ Cepstrum coefficient, $k$ is the dimensionality of the model.

- V-Order and Log Detector (V and LOG). - V and LOG yield an estimation of the exerted muscle force. The $V$ is defined as

$$
V=\sqrt[v]{E\left\{\left|x_{k}\right|\right\}^{v}}
$$

where $E$ is the expectation operator applied in one analysis window on the samples.

The LOG is defined as

$$
L O G=e^{\frac{1}{N} \sum_{k=1}^{N} \log \left(\left|x_{k}\right|\right)}
$$

- EMG Histogram (HIST). It is an extension of ZC and WAMP, it measures the frequency with which the EMG signal reaches multiple amplitude levels.

The TD features are the most used in literature, since they are the most intuitive, relatively easy to implement, and require less computational resources. Nevertheless, in literature also other features have been investigated, as frequency domain and time-frequency features [20] [22], as spectral measure, Fourier transform, wavelet transform and wavelet packet transform.

To classify the EMG signals and assign a label to different movements a classifier is needed. A classifier is capable of exploiting the information received from the extracted features and decide to which class the information belong. The most used classifiers in this field are linear discriminant analysis (LDA), support vector machine (SVN), and hidden Markov model [16]. The LDA, respect to the other classifier, is more easy to implement and to train. It has been shown that the LDA classifier does not compromise the classification accuracy [24], 
compare with the others [16], hence it is the most used in literature. To classify the data, part of it is used to train the classifier and the remaining part is used as test set to evaluate the classification accuracy. The performance of a classifier is usually evaluated measuring the classification accuracy (CA), which is defined as

$$
C A=\frac{\text { Number of correct classifications }}{\text { total number of classifications }} \times 100 \%
$$

\subsection{Challenges in the use of surface electrodes in EMG pattern recognition}

The pattern recognition methods proposed in literature, for control of upper limb prosthesis, are based on EMG signals acquired with surface electrodes. The number and configuration of the electrodes has been also object of investigation. It has been demonstrated that the performance of the EMG pattern recognition can improve increasing the number of channels (i.e. electrodes) [16], [25].The number of channels depend on the number of residual muscles of the amputee and on the number of DoF that are allowed to the prosthesis. In the studies that have been done on this topic the number of the electrodes varies usually between 4 and 16, for transradial amputee (amputation through forearm). In [26] it has been found that no significant increase in classification accuracy resulted from adding more than 4 channels, using a symmetrical channel subset. In case of amputee with a higher level of amputation, this is not applicable and the number can vary a lot depending on the anatomy of the shoulder (in case of patient with target muscle reinnervation) or of the residual limb [16].

The classification accuracy that can be reached with pattern recognition algorithms is very high, most of the time over the $95 \%$. Nevertheless, this is not the only factor that influences the usability of prostheses. The robustness and the reliability for upper limb prostheses are characteristics of extreme importance. The signals that control the prosthesis have to be 
reliable, not affected by any kind of noise, moreover the prosthesis need to be robust, meaning that the electrodes displacement due to donning and doffing should not influence the functioning [20]. The repositioning of the prosthesis is one of the main issues, in pattern recognition algorithms, and it is correlated to the use of surface EMG electrodes. Indeed, a potential shift can occur in case of the donning and doffing of the prosthesis or because of loading and positioning of the limb. The shift of the electrodes can affect the classification accuracy, the robustness and the long-term reliability of pattern recognition algorithms [27]. The classification accuracy can be influenced also by the malfunctioning of some electrodes during the use of the prosthesis. The malfunctioning can be caused by power line interference or by poor contact between skin and electrode. The fault of only one electrode can cause degradation in classification accuracy, and to reactivate the correct operation of the prosthesis it is required a re-training of the classifier. These problems have been taking on in the last few years in literature. In [28] the problem of the shift of the electrodes has been carried on, proposing to train the classifier with signals from all the expected displacement locations and suggesting that the use of AR (AutoRegression) coefficient as features may be more robust. This procedure has some drawbacks as the plausible displacement locations have to be identified in advance, and it results time consuming, since the classifier has to be trained over all the possible locations of shift. In [18] have been studied the EMG features that can offset the impact of the electrodes displacement. The authors have found that a combination of three features can improve the performance, but cannot solve the problem. It has been also considered if the orientation and size of the electrodes can influence the classification accuracy and reduce the influence of the shift [29]. The authors concluded that longitudinally oriented electrodes perform better than transverse-oriented, without and with the shift, moreover they found that larger electrodes can reduce the sensitivity to the shift, but they are performing worse in the original locations. Another important finding of this study concerns the direction of the shift. Shifts that are perpendicular to muscle fiber are affecting more the pattern recognition algorithm than parallel shift. To reduce the shift sensitivity also the influence of interelectrode 
distance has been considered. In [31] it has been investigated the optimal distance, the electrode configuration, along with EMG features set, that can be effective against the shift problem. The study found that a interlectrode distance $(4 \mathrm{~cm})$ larger than usually applied, with a combination of longitudinal and transverse locations can reduce the sensitivity to shift. Moreover it has been asserted that for transradial amputees it is more advisable to use four to six electrodes and that the features set that reduce more the shift effect is TDAR [27], a combination of TD and AR features.

All the studies referenced previously in this paragraph proposed features and configuration of electrodes to reduce the sensitivity to shift of the electrodes, moreover they are exploiting a small number of electrodes. Nevertheless, all of them highlight the impossibility to eliminate the undesired effect of the electrodes displacement, which is considered one of the main reasons for the lacking of diffusion of upper limb myoelectric prostheses, controlled with pattern recognition algorithms. Taking into account these findings a new solution has been proposed. A novel method for pattern recognition of EMG signals based on High Density (HD) EMG recording have been investigated. This method allowed also to eliminate malfunctioning channels without re-train the system. The new method will be described in the next chapter. 


\section{Spatial correlation of High Density EMG signals \\ as new robust features for myocontrol}

The research on myoelectric control has usually focused on a small number of channels. A number of studies stated that between four and six channels are enough to get good classification accuracy (>95\%). This choice has been justified by the reduced computational load and by the simplicity of use. The two major issues of pattern recognition algorithms, the shift and the malfunctioning of the electrodes, have been identified. Many studies to solve these problems have been conducted. Nevertheless, they have not been completely solved and consequently pattern recognition algorithms are not yet implemented in the upper limb prostheses commercially available. For these reasons, in the following paragraphs it has been proposed a novel pattern recognition method, which exploits the spatial correlation of the HD EMG signals. The work presented in the following of this chapter [30] has been published by me as first author on IEEE transaction of Neural Systems. Therefore text or results reproduced form this manuscript are not cited explicitly in the chapter. This is the first study that uses spatial correlation and HD EMG for the control of upper limb prostheses.

\subsection{High Density sEMG in myoelectric control}

High Density surface EMG electrodes can record EMG signals from multiple locations, closely spaced, over the skin surface. They usually have the configuration of a grid and are embedded in flexible silicon rubber or in clothing [32] (Figure 3). This type of electrodes has been used to identify individual motor unit activities from non-invasive recording [32]. HD EMG matrices are characterized by two spatial and one temporal dimensions, they can be used to investigate the muscle activity pattern and to draw topographical maps of EMG amplitude [33]. The limitation of this type of EMG recording is the amount of data available and consequently the computational load. Recently this technology has been considerably improved and with the development of powerful microprocessors, it has been proposed as 


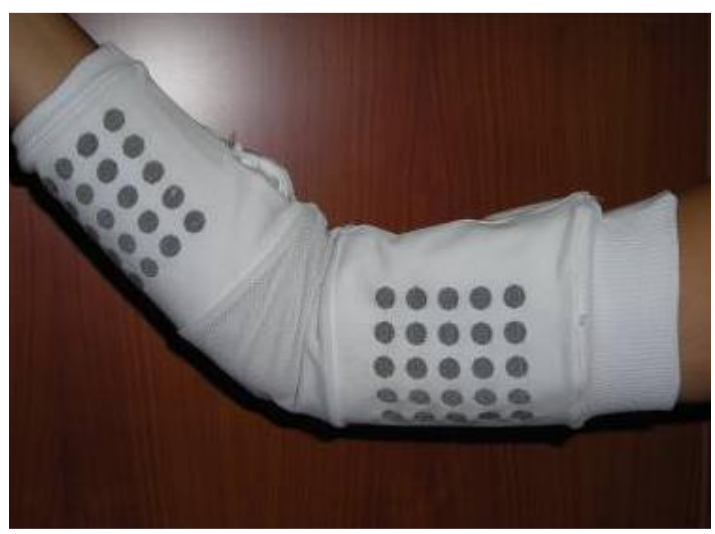

(a)

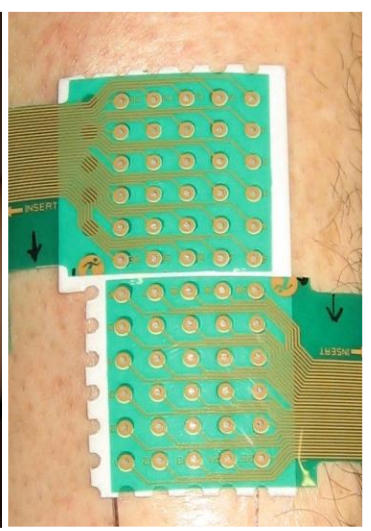

(b)

Figure 3. Example of HD EMG. (a) Texile HD EMG. (b) Silicon HD EMG.

input signal for prosthetic control for transradial amputees [34] [35]. Their use was limited to identify the subset of optimal electrode position [34], for the estimation of the kinematics of the joint wrist/hand [35], or to demonstrate the robustness of the synergy method across some different channel configuration [36].

The HD EMG have been used also on TMR patients. HD EMG signals have been recorded from TMR patients with the intent to improve the myoelectric control [37] [38]. The number of channels used in these two studies are 115 and 128 respectively. HD EMG signals (128 channels) have been used also in TMR patients to extract neural control information from reinnervated muscles and to determine the optimal placement of the electrodes [39]. In [40] have been used up 448 channels on TMR patients to extract single motor unit spike trains, providing the neural code that underlined tasks of the phantom limb.

In any of the studies mentioned the spatial information of EMG signals coming from the entire matrix of electrodes have been exploited to improve the myoelectric control. 


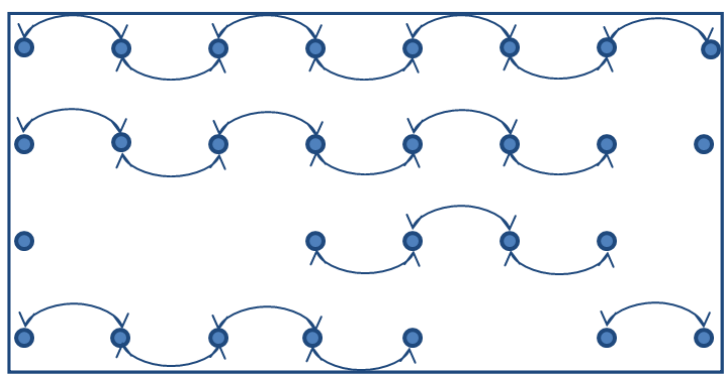

(a)

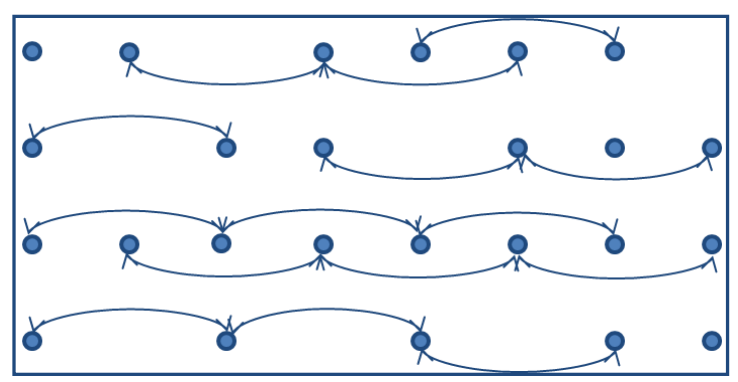

(b)

Figure 4. Identification of all pairs at distance (a) $h=1$ and $(b) h=2$ apart in the direction of the $x$ axis for a representative grid.

\subsection{Spatial correlation - The variogram}

A HD matrix of EMG electrodes can be considered as a grid of sensors that record data that are spatially correlated. The spatial correlation of data is based on the concept that samples recorded in proximity are likely to have similar values and to vary in the same way. The spatial correlation of data has been exploited mainly in the telecommunication field. It has been utilized in the field of dense sensors networks [41][42] for different scopes, as compression of data and routing [43][44], to cover possible holes in the networks [41], or for collaborative MAC layer [45]. In a sensor network the data from sensors that are closely spaced are spatial correlated. In geostatistic, the variogram (also called semivariance), is a statistical measure of the spatial correlation. The variogram is a graph of the semivariance against the distance between pairs of locations in the network. It can be defined as a function that describes the spatial correlation between observations. The main concept of the variogram is that the value of a variable $z$ depends on the location in which it is observed $(x)$. The value of $z$ at $x, z(x)$, is auto-correlated with the value $z(x+h)$, where $h$ (the lag) is the distance in the direction $\mathrm{x}$. Considering the random variables $Z(\boldsymbol{x})$ and $Z(\boldsymbol{x}+\boldsymbol{h})$ at the points $\boldsymbol{x}$ and $\boldsymbol{x}+\boldsymbol{h}$, the variogram function is defined as follows:

$$
2 \gamma(\boldsymbol{x}, \boldsymbol{h})=E\left\{[Z(\boldsymbol{x})-Z(\boldsymbol{x}+\boldsymbol{h})]^{2}\right\}
$$


The variogram $\gamma(\boldsymbol{x}, \boldsymbol{h})$ is a function that relates the semivariance to the spatial separation, assuming a multi-dimensional space and a stochastic process. The lag $\boldsymbol{h}$ and the location $\boldsymbol{x}$ are written in bold to denote that now they are vectors with a magnitude (distance) and orientation.

The variogram function $\gamma(\boldsymbol{x}, \boldsymbol{h})$ in equation 3.1 depends on both $\boldsymbol{x}$ and $\boldsymbol{h}$. The estimation of such function requires a certain number of realizations of the pair of random variables, that are not available in practical application. To overcome this issues the Matheron's intrinsic hypothesis (a weaker assumption than the second order stationarity) [46] has been assumed. The Matheron's intrinsic hypothesis states that the variogram function depends only on the distance vector $\boldsymbol{h}$ (modulus and direction) and not on the location $\boldsymbol{x}$. It is then possible to define an estimator of the variogram, which is known as experimental variogram:

$$
\hat{\gamma}(\boldsymbol{h})=\frac{1}{2 n(\boldsymbol{h})} \sum_{i=1}^{n(\boldsymbol{h})}\left[z\left(\boldsymbol{x}_{i}\right)-z\left(\boldsymbol{x}_{i}+\boldsymbol{h}\right)\right]^{2}
$$

where $z\left(\boldsymbol{x}_{i}\right)$ is a measurement taken at location $\boldsymbol{x}_{i}$ and $n(\boldsymbol{h})$ is the number of experimental pairs $h$ units apart in the direction of the vector $\boldsymbol{h}$ [46]. In this notation, $z$ represents a specific realization (the measurement) of the stochastic process $Z$. When considering a grid, the variogam is a function of the discrete distance. Figure 4 reports an example of measurements recorded on a grid. The circles represent the positions of the measurements for which the experimental variogram has been calculated. Figure 4(a) shows all the pairs at $h=1$ apart in the direction of the $x$ axis, while Figure 4(b) shows all the pairs at $h=2$ apart in the direction of the $x$ axis. In the example of the Figure 4, there are missing measurement points in the grid and this has been taken into account in the assessment of the pairs with a certain distance.

The size and the configuration of the sample can influence the reliability of the variogram. The estimation of the variogram is more precise when the number of the spatial measurement points (sample) is larger [46]. The maximum lag should be limited to half the 


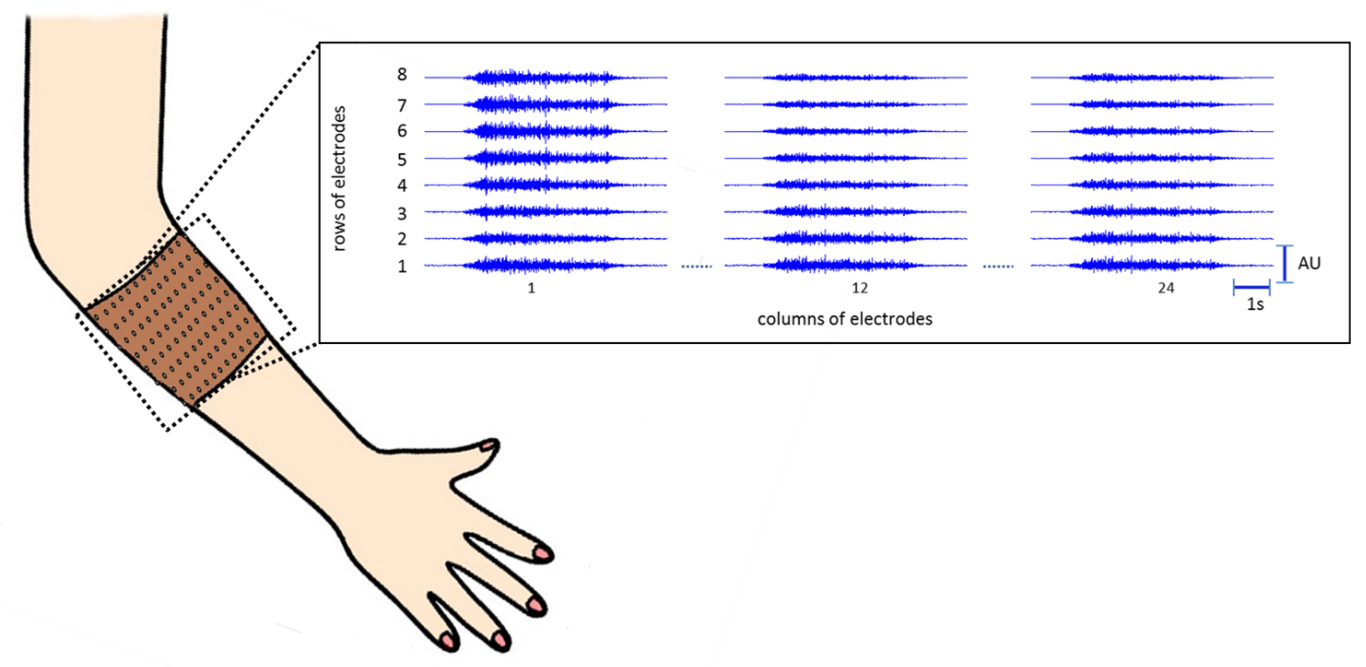

Figure 5. Positioning of the HD EMG matrix and EMG signals. Reused and modified with permission [30] (C) 2015 IEEE.

extreme distance in the sampling domain and the minimum number of pairs for the estimation of the variogram that is usually accepted is approximately 50 [47]. The experimental variogram is usually evaluated for the main directions. The variogram is said anisotropic when the spatial variation is not the same in all directions. In this case the semivariance can be represented as a surface.

\subsection{Methods}

To evaluate the robustness to the shift and to electrode number of the algorithm proposed, EMG data have been acquired from human subjects.

The subjects of the experiments were seven able-bodied (three males and four females) and one uni-lateral trans-radial traumatic amputee. The average age of the able-bodied subjects was $29.0 \pm 4.7$ years. The recruited amputee was 78 years old (53 years post-amputation). The protocol has been approved by the ethics committee of the University Medical Center Göttingen. 
The EMG signals were collected with an adhesive grid of 192 electrodes placed on the circumference of the forearm (as shown in Figure 5), starting at the ulnar bone. The grid or matrix of electrodes consists of 8 rows and 24 columns, with interelectrode distance of 10 $\mathrm{mm}$ in both directions. Before placing the matrix, the skin of the subjects was slightly abraded and cleaned with alcohol. The EMG signals were amplified (EMG-USB2, OT Bioelettronica, Turin, Italy) with a gain of 500, sampled at $2048 \mathrm{~Hz}$ and A \D converted on 12 bits.

The able-bodied subjects had three motion trackers (XSENS Technologies, NL), they were positioned on the back of the hand at the wrist, and below the elbow. Finally, a reference electrode was positioned at the wrist.

The able-bodied subjects were asked to perform a combination of 4 Dof (wrist flexion, wrist extension, radial deviation, ulnar deviation, forearm pronation, forearm supination, hand open, hand closing) plus the rest position, for a total of 9 tasks (classes). In the first phase of the experiment the subjects were trained. An assistive software provided the direction and time of the movements on a computer screen. The starting position was with the arm along the body in resting position, with the palm inwards. Four repetitions of each movement were recorded, for a total of four trials for each subject.

Because of the short stump of the amputee subject, the matrix has been modified. Two rows have been removed. In this case the grid was made by 6 rows and 24 columns, for a total of 144 electrodes. The matrix was positioned on the stump, the reference electrode on the elbow. The three motion trackers were placed on the contralateral arm.

The subject performed mirrored movements, thinking to replicate the tasks of the able-limb with the phantom limb.

Before the extraction of the features for the pattern recognition algorithm, the raw EMG signals were preprocessed. A fourth-order Butterworth bandpass filter $(10-500 \mathrm{~Hz})$ was applied. The static part of the signal was identified with the data provided by the tracking 
(a)
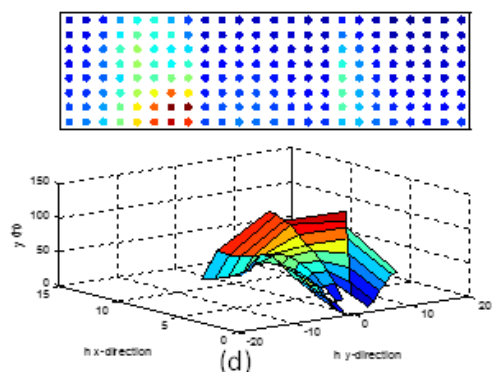

(d)
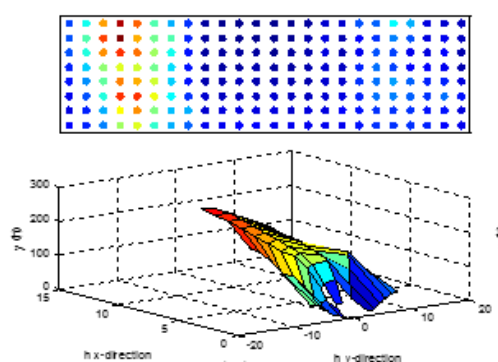

(g)
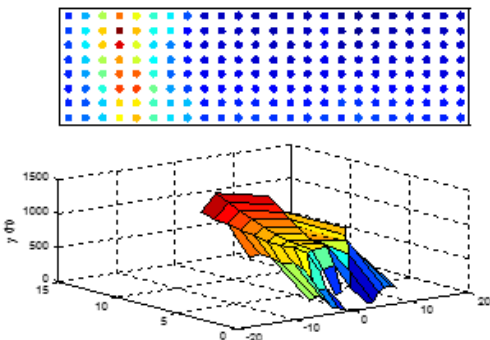

(b)
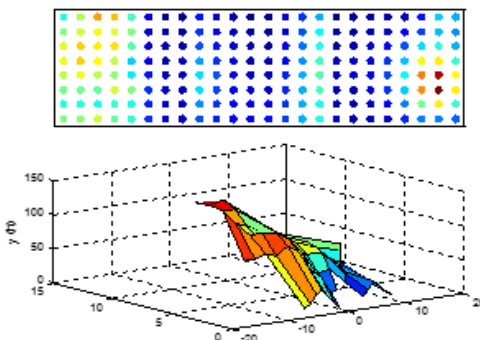

(e)
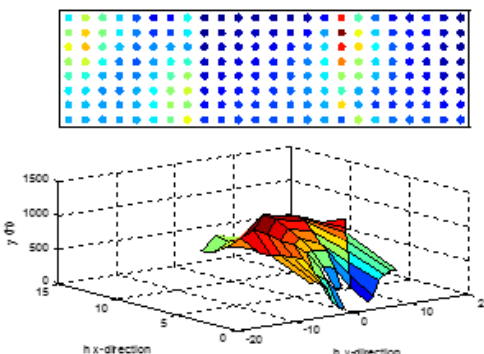

(h)
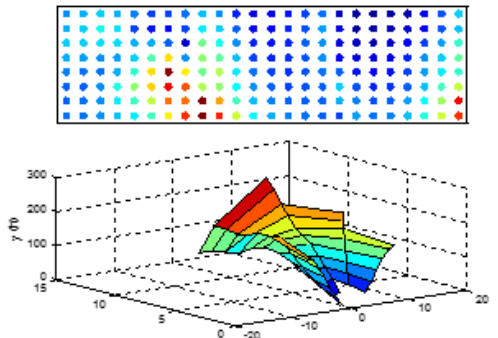

(c)
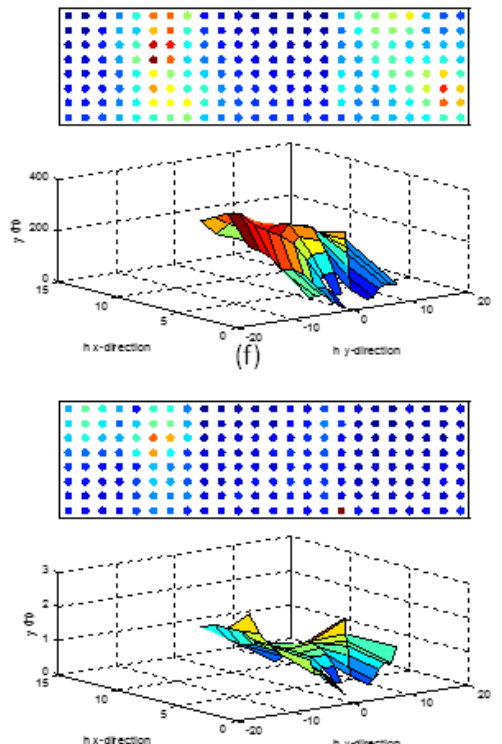

(i)

Figure 6. Representation of EMG RMS values on a matrix of 192 electrodes and the corresponding experimental variogram for one normally-limbed subject. (a) Wrist flexion, (b) wrist extension, (c) forearm supination, (d) forearm pronation, (e) radial deviation, (f) ulnar deviation,(g) hand open,(h) hand closed, (i) rest. For the RMS, the colors are normalized so that red represents the maximum value and dark blue the minimum. The same color code is used for the variograms. Reused with permission [30] C 2015 IEEE.

system. The static part corresponded to a window of 4500 samples ( $\sim 2.2 \mathrm{~s})$. The center of the static part matched to the center of each movement. The RMS value of the EMG signal was calculated over 30 intervals for each window, which means over 150 samples (50 ms). Considering that the grid is regular with normalized distance equal to 1 , the RMS value was calculated for each signal of the matrix. The RMS values for each window of 150 samples were used to calculate the experimental variograms, using the equation (3.2). The RMS of each signal of the grid was $z\left(\boldsymbol{x}_{i}\right)$, considering the maximum lag as half of the longer 


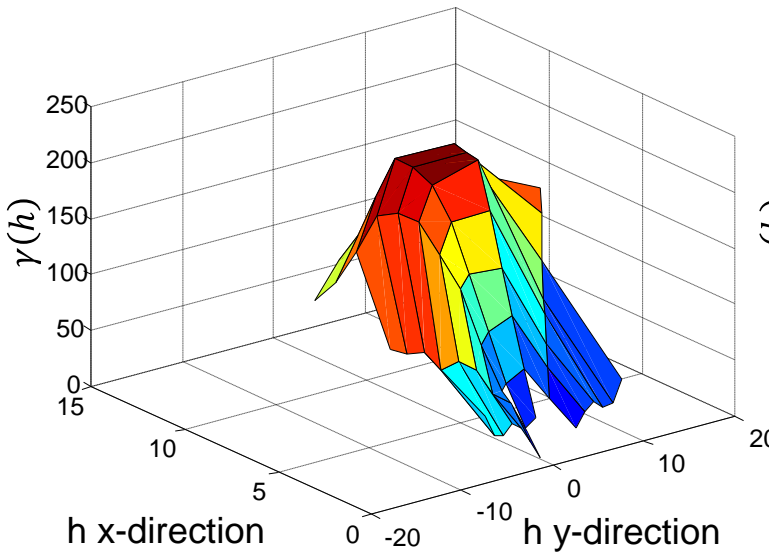

(a)

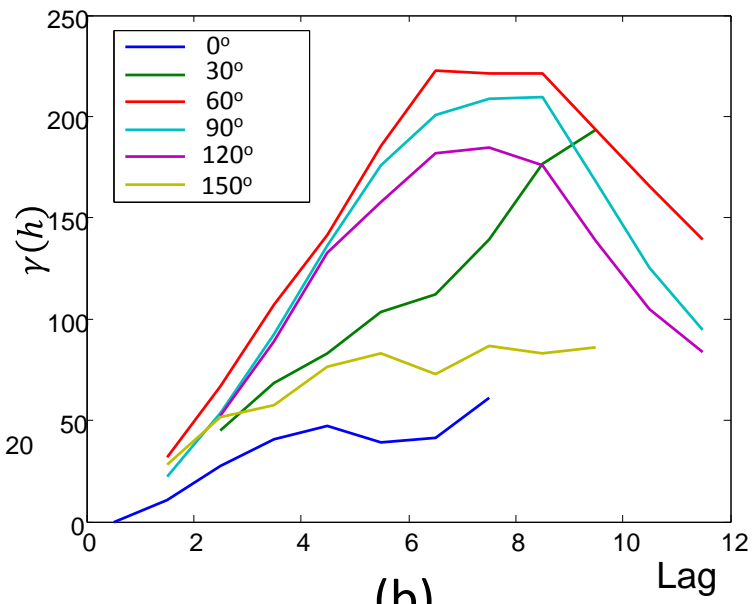

(b)

Figure 7. Experimental variagram, case of wrist flection for an able-bodied subject. (a) Directional variogram; (b) Cartesian representation of the directional variogram curves. Reused and modified with permission [30] (C) 2015 IEEE.

dimension of the grid, which was 24. Moreover, since it has been noticed that the variogram in different directions did not have the same trend, it was computed the anisotropic variogram, which includes six directions in the range $0-180$ degrees. The anisotropic experimental variogram is represented as a surface. Figure 6 shows the RMS values of the EMG signals and the anisotropic experimental variogram for an able-bodied subject while performing the 9 tasks.

The color of RMS values is normalized so that the red represents the maximum value and the dark blue the minimum. The same color code has been used for the experimental anisotropic variograms.

The points of the six curves of the anisotropic variogram in Cartesian coordinates, have been used as features for classification. The number of points can vary depending on the direction of the variogram and on the value of the maximum distance. In Figure 7 an example of an isotropic variogram related to a wrist flexion for an able-bodied subject. In 


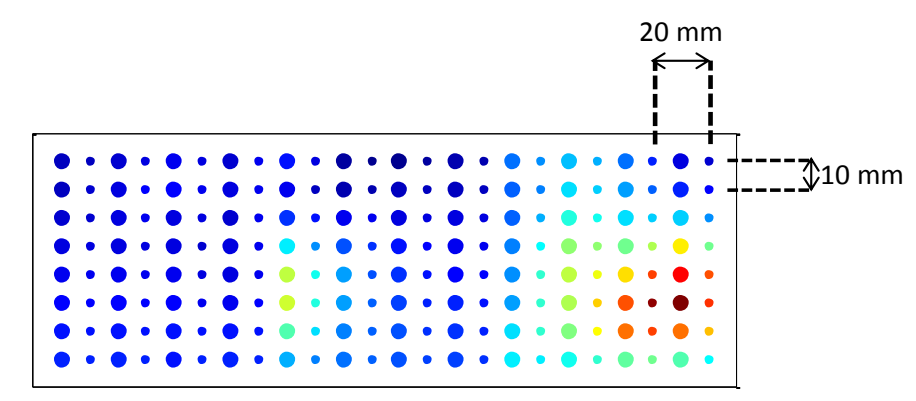

(a)

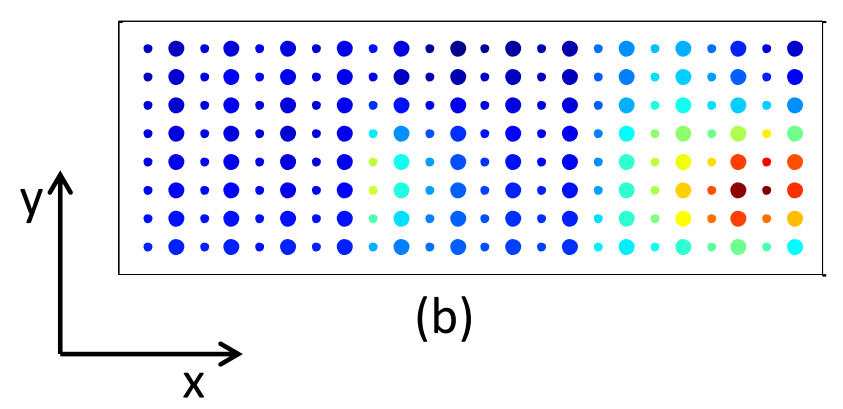

Figure 8. Shift inwards/onwards ( $8 \times 12$ electrodes). The bigger circles are the electrodes used for training, while the smaller circles are the electrodes used for testing. (a) Shift onwards (b) Shift inwards. Reused and modified with permission [30] ๔ 2015 IEEE.

figure $7 \mathrm{a}$ the directional variogram, while Figure $7 \mathrm{~b}$ shows the representation of the anisotropic variogram in Cartesian coordinates.

A Support Vector Machine (SVM) [48] has been chosen as a classifier. The SVM scope is to produce a model, based on the training data, which predict the classes labels of the test data. The multiclass problem was solved with a one-versus-one (OVO) approach. The linear kernel was chosen since in preliminary analysis it was noticed that there was a better classification accuracy with it. The results obtained with the SVM classifier and the variogram features were compared with the most used classifier e features. The classifier is the linear discriminant analysis (LDA), while the features sets chosen are: RMS, TD (mean absolute value, zero crossing, slope sign changes, waveform length) and Time-Domain Autoregressive (TDAR). The RMS as feature has been selected since is a very simple method used in a large number of studies. The TD features have been considered in many studies on 


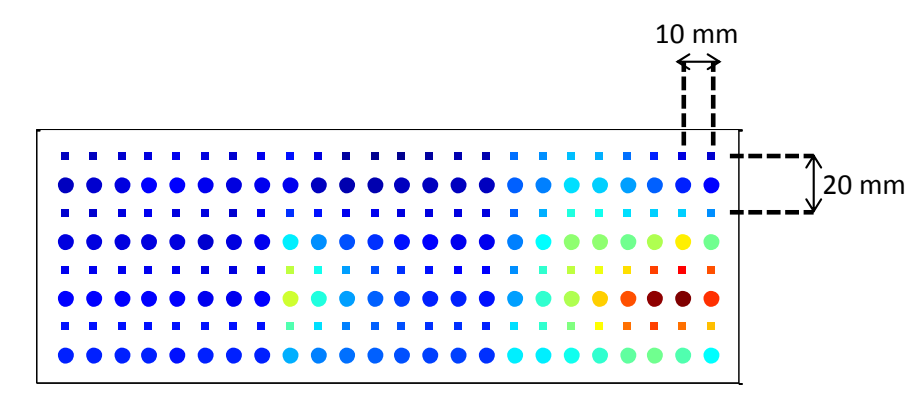

(a)

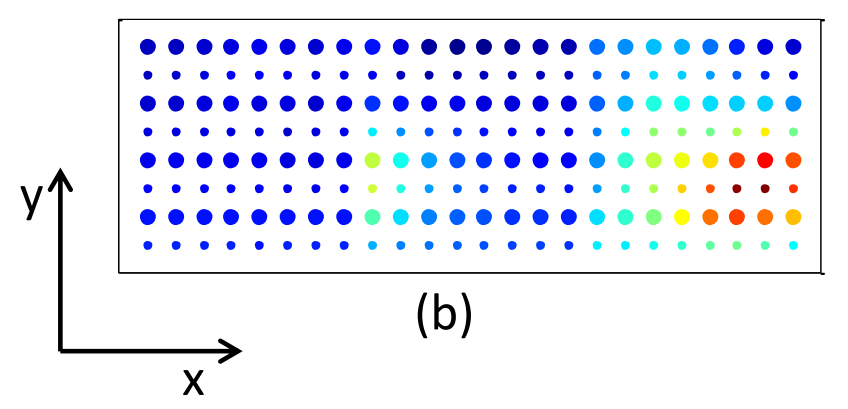

Figure 9. Shift upwards/downwards (4×24 electrodes). The bigger circles are the electrodes used for training, while the smaller circles are the electrodes used for testing. (a) Shift upwards. (b) Shift downwards. Reused and modified with permission [30] C 2015 IEEE

electrodes shift [29], moreover they are considered reliable in real time control [49]. Finally, the TDAR features have been considered as considered the most effective to reduce the shift of the electrodes [31]. The RMS, TD and TDAR have been calculated over 150 samples, the number of samples for which the window was shifted forward was set to 50 and the number of samples per movement, to avoid over-shoot of windows into the next movement, was set to 4500 , as for the variogram.

The classification accuracy has been tested for several scenarios. It has been evaluated considering all the electrodes of the matrix, smaller subsets of electrodes, shifts of the electrodes in the transversal and longitudinal directions of the muscle fibers, and in presence of noise. The shifts have been simulated in both directions, considering alternatively, half of the columns and half of the rows, for training, and the other half for testing as explained in the Figure 8 and Figure 9. It has been considered a shift of $10 \mathrm{~mm}$, 


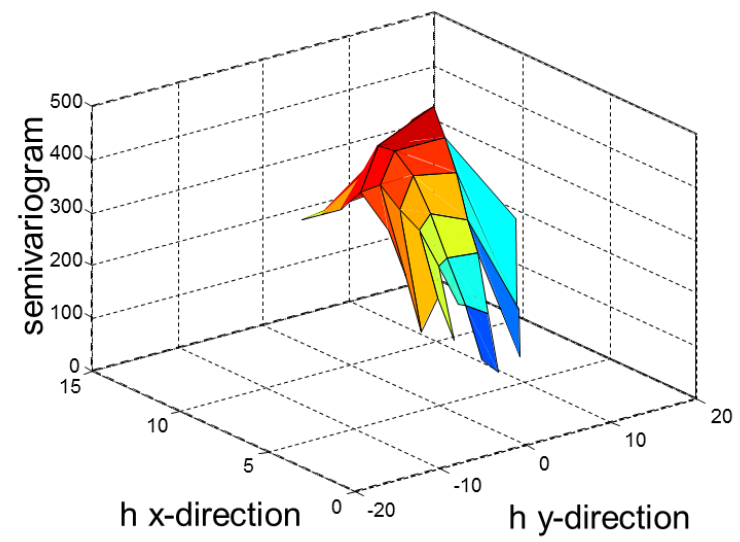

(a)

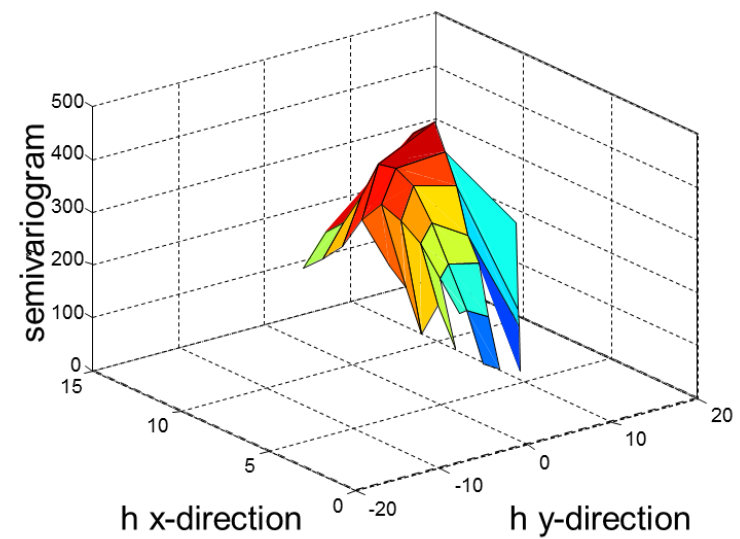

(b)

Figure 10. Example of variograms used for shift onwards, case of wrist flexion. (a) Directional variogram used for training; (b) directional variogram used for testing. ). Reused and modified with permission [30] (C) 2015 IEEE

considering that this value has been evaluated as the most likely in clinical situations [29]. Figure 8 shows the shift inwards/onwards. The bigger circles are the electrodes used for training, while the smaller circles represent the electrodes used for testing. The shift in this case is the direction of the $x$ axis and the distances between the electrodes is $20 \mathrm{~mm}$ in the $x$ direction and $10 \mathrm{~mm}$ in the $y$ direction. Figure 9 shows the shift upward/downwards, same as Figure 8 the bigger circles are the electrodes used for testing, while the smaller are the one used for training. The shift is in the direction of the $y$ axis. The distances between the electrodes are respectively $10 \mathrm{~mm}$ in the $\mathrm{x}$ direction and $20 \mathrm{~mm}$ in the $\mathrm{y}$ direction.

In Figure 10 are shown the experimental variograms used for classifications in case of shift onwards. In Figure 10 a the experimental variogram used in the training phase, while in Figure $10 \mathrm{~b}$ is the variogram used for testing.

In the last part of the study the presence of noisy channel has also been considered. The noise has been simulated as Gaussian distribution and same standard deviation of the selected EMG signal. The channels with noise have been selected randomly and varied between 1 and 95 with a step of 5 . For each subject it was repeated for 10 times. In one case the noisy channels were used for testing, while in the other case they were eliminated 
before the classification considering that was possible to identify them [50]. In all noisy cases considered there were no re-training of the system. The training was done with three trials including all the 192 electrodes, while the testing has been done with the noisy channels in one case or with a reduced number of channels in case they were removed. The same tests have been done also on the amputee, considering that in this case the matrix had 144 electrodes ( 6 rows and 24 columns). The results obtained from these tests have been compared with these obtained from the other classifiers and features, in the same conditions. 


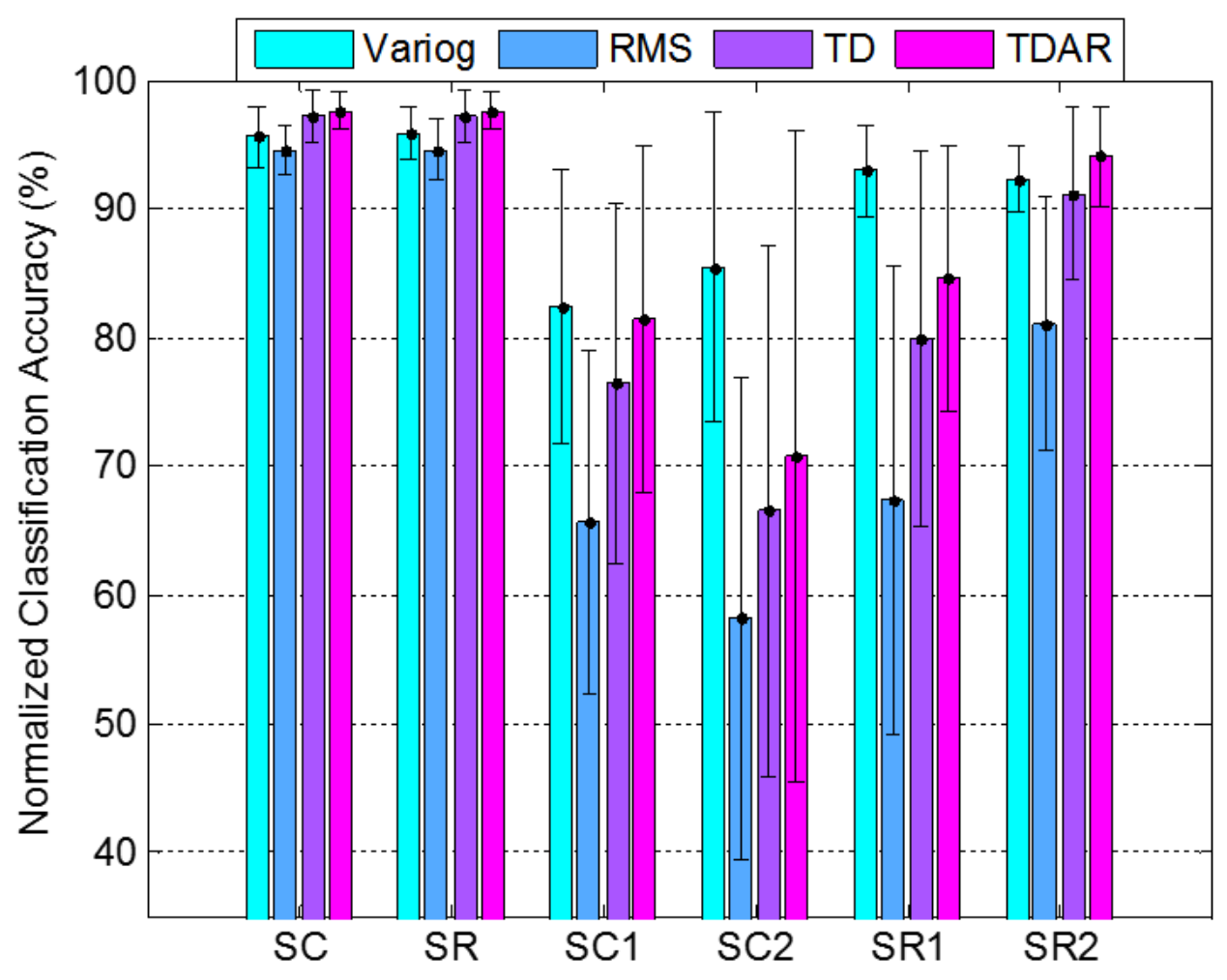

Figure 11. Results on 7 abled-bodied subjects. Average classification accuracy for four classification methods: Variogram with SVM (Variog), RMS with LDA (RMS), TD with LDA (TD), TDAR with LDA (TDAR). The accuracy has been calculated for six cases: the first and the second case (SC and SR) are half of the matrix in no shift positions. SC is the mean and standard deviation of the two configurations used for the shift in the $x$ direction, SR is the mean and standard deviation of the two configurations used for the shift in the $y$ direction. The other 4 cases are the shifts. Mean and standard deviation of the classification accuracy normalized with respects to the accuracy obtained by the grid of 96 electrodes without shift. The shifts considered are the following: shift inwards of the columns (SC1), shift onwards of the columns (SC2), shift upwards of the rows (SR1), shift downwards of the rows (SR2). Reused and modified with permission [30] (C) 2015 IEEE.

\subsection{Results and discussion}

\subsubsection{Effect of electrodes shift}

The classification accuracy has been calculated for the four methods described in previous section (variogram, RMS, TD, TDAR) for 9 classes and averaged over the 7 able-bodied subjects. In Figure 11 are shown the final results for six configurations. SC and SR represent 


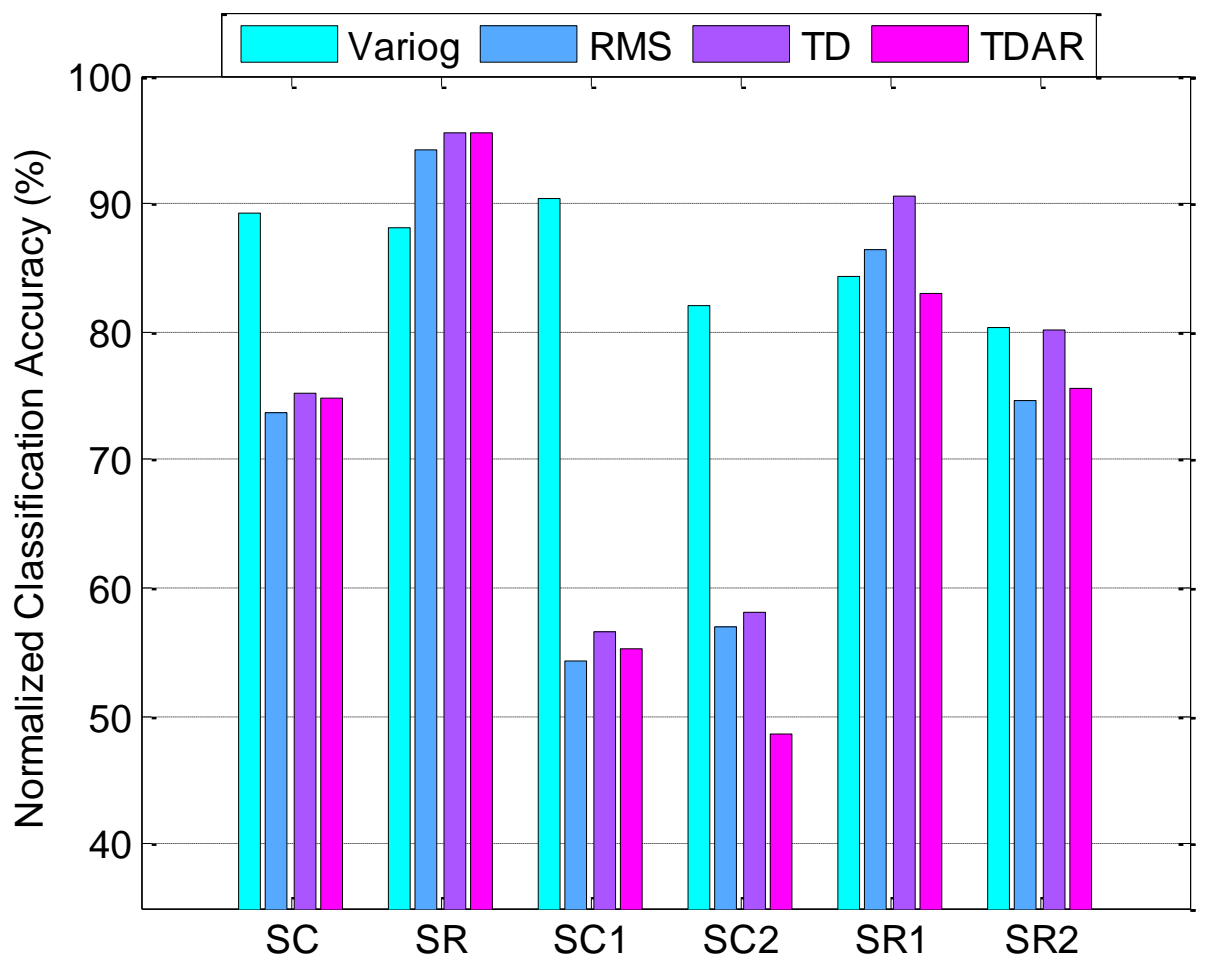

Figure 12. Classification accuracy for an amputee subject over 7 classes. SC and SR are the configurations with half matrix (72 electrodes) with no shift. SC1, SC2, SR1 and SR2 are the shift configurations as in Figure 8. Reused and modified with permission [30] C 2015 IEEE.

the case with 96 electrodes and no shift. SC is the mean and standard deviation of the two configuration used for shift in the $\mathrm{x}$ direction, while $\mathrm{SR}$ is the mean and standard deviation of the two configuration used for the shift in the $y$ direction as depicted respectively in Figure 8 and Figure 9. For all the four methods (variogram, RMS, TD, TDAR) the classification accuracy was 95\%, according with previous studies [28] [34].

The remaining four cases are the mean and standard deviation of the normalized classification accuracy of the shifts. The classification accuracies have been normalized respect to the accuracy obtained by the grid of 96 electrodes without shift. SC1 represent the case of the shift inwards of the columns, SC2 the shift onwards of the columns, SR1 the shift upwards of the rows, and SR2 the shift downward of the rows. In these cases, the 
variogram method (Variog) resulted more robust to the shift respect the classic features in both, transversal and longitudinal direction, except for SR2.

The results obtained presented here have been obtained considering all the channels, without discarding any channels that can be malfunctioning. The values of the classification accuracies of the variogram for the four shift cases (SC1, SC2, SR1, SR2) are closer to the reference condition respect to RMS and TD. Variog is also comparable in SC1 respect to the TDAR, which is the recommended method for reducing the shift [31]. Slightly worse is the accuracy of the variogram in SR2 respect to TDAR. Looking at the standard deviation of the shifts cases, it is possible to notice that in the case of the variogram it results lower that the other methods.

This study confirms also that the electrodes shift in the longitudinal direction of the muscles fibers influence less the classification accuracy than the shift in the transversal direction [31]. The Variog approach resulted robust to both shifts and with an accuracy of $~ 90 \%$ with respect to baseline for the shift in the longitudinal direction and $\sim 80 \%$ for the shift in the transversal direction. Moreover, the variogram provided similar performance for the four shift considered, contrary to the other methods that had more variable results across conditions. The results related to the shifts have been obtained without training the system on all possible displacements sites as in [28], and also without choosing the optimal electrodes location [34][39]. The electrodes in this study were just organized in a regular grid and placed on the circumference of the forearm. The training and the testing has been done on the configurations depicted in Figure 8 and Figure 9. Nevertheless, the final results were comparable to those shown by Hargrove et al. [28] with extensive training in multiple electrode displacements.

The shifts considered here are all of $10 \mathrm{~mm}$. This has been considered as a plausible value of variation in electrodes position when donning and doffing the prosthesis in clinical studies [27]. 


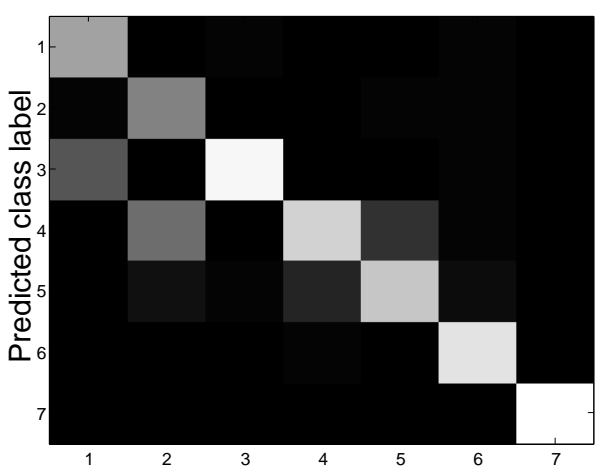

(a)

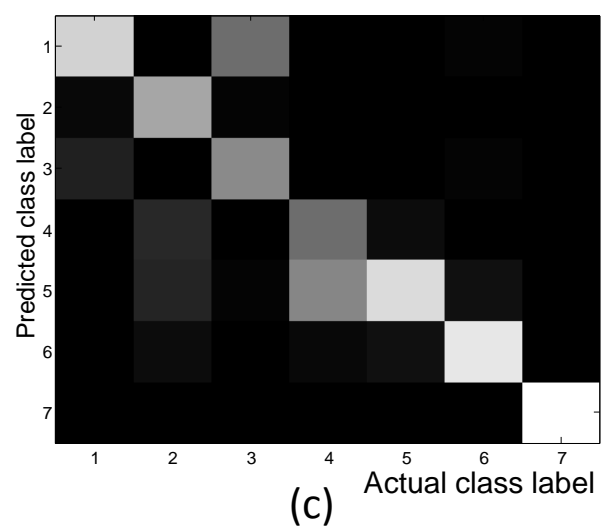

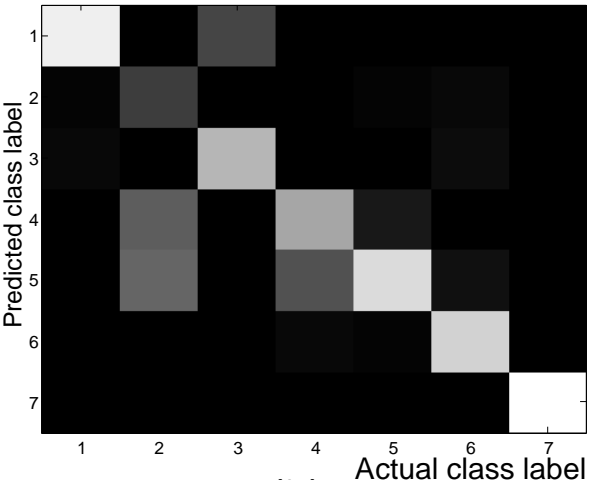

(b)

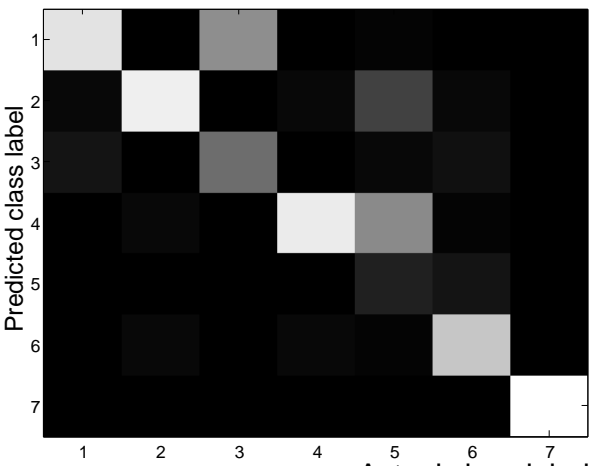

(d)

Figure 13. Confusion matrices of the four shifted configurations with the Variog method for the amputee subject over 7 classes: wrist flexion (1), wrist extension (2), radial deviation (3), ulnar deviation (4), forearm pronation (5), forearm supination(6), rest (7). (a) SC1; (b) SC2; (c) SR1;( d) SR2. Reused and modified with permission [30] (C) 2015 IEEE.

The statistical differences among the four methods and grid configuration on the normalized values, have been analyzed with a two-way ANOVA. Both factors had 4 levels (method: Variog, RMS, TD, TDAR; grid configuration: SC1, SC2, SR1, SR2) and both factors were statistically significant $(p<0.05)$, with no interaction.

In the next figure (Figure 12) are summarized the results for the amputee subject. The no shift case has 72 electrodes (SC and SR). The shift cases are also normalized respect to the reference matrices (SC and SR). In the SC (72 electrodes without shift) configuration the classification accuracy of the Variog ( 90\%) was greater than all other methods ( 75\%) although it was slightly worse (by $\sim 8 \%$ ) in the SR configuration. The accuracies of shifts on 


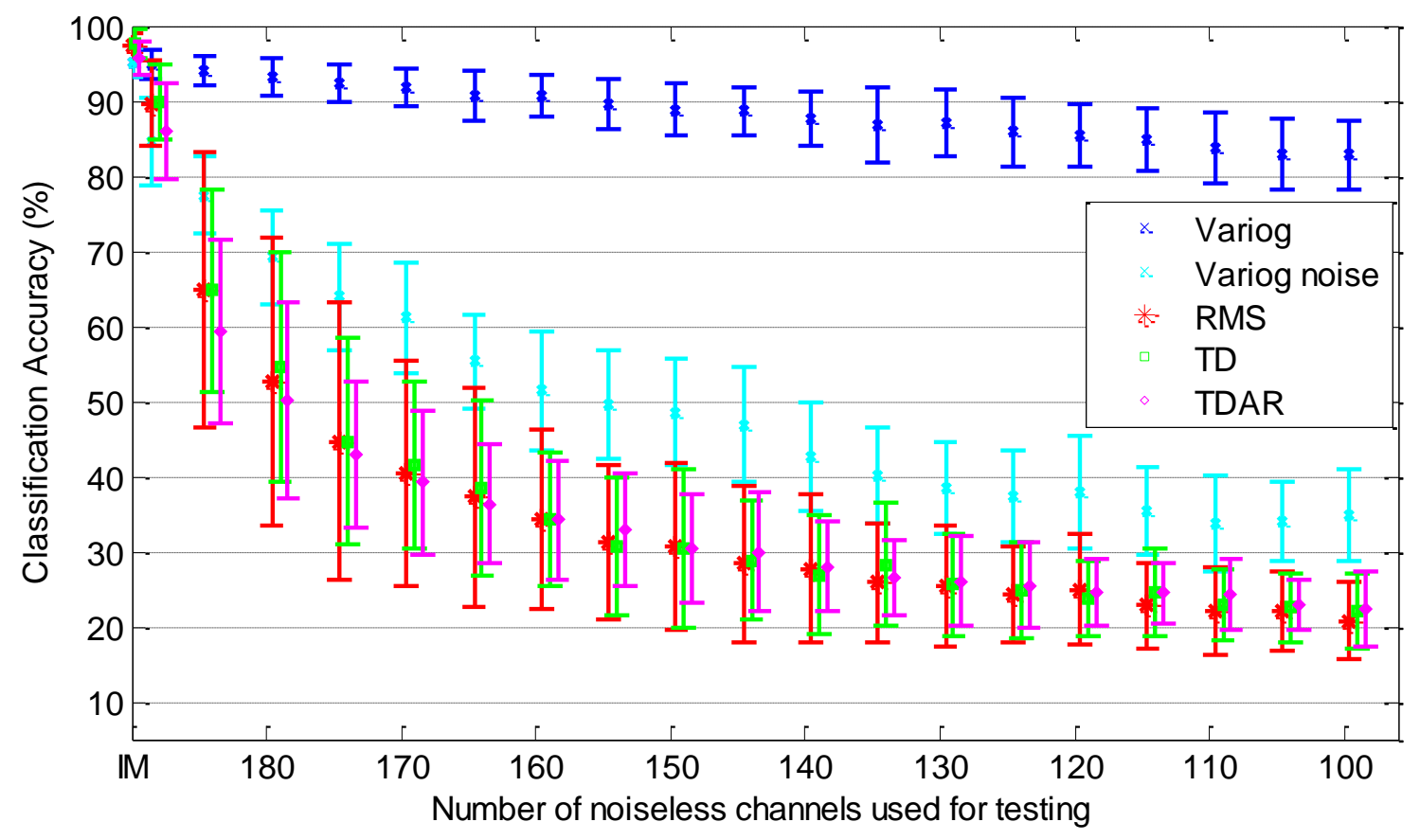

Figure 14. Averaged classification accuracy and standard deviation (9 classes) for 7 able-bodied subjects as a function of the number of channels used in the classification test for Variog (dark blue) and as a function of the noiseless channel on the other 4 cases (Variog noise, RMS, TD,TDAR). Reused and modified with permission [30] (C) 2015 IEEE.

the transversal direction of the muscle fibers (SC1 and $\mathrm{SC} 2$ ) resulted comparable with the cases with no shift for the Variog methods. While the classical methods perform worse. The shift by rows (SR1 and SR2), have similar results for all methods. For the amputee subject the shift in the longitudinal direction and the shift in the transversal direction show similar classification accuracies for the variagram, contrary to the able-bodied subjects who showed better results with longitudinal shifts. The explanation can be found in the fact that the intact matrix, in the case of the amputee, had 2 rows less, 6 instead of 8, respect to the able-bodied subjects. This imply that there is less spatial information in the direction of the $y$ axis. The variogram performed certainly better than all the other methods in the shift inwards/onwards, even better than TDAR, in SC, SC1 and SC2. 
For the amputee a further analysis has been conducted with the confusion matrix (Figure 13). In this figure can be noticed that the rest position has been always classified correctly (label 7). The most misclassified for the shift in the transversal direction case was the wrist extension (label 2, in Figure $13 \mathrm{a}$ and b). For the case of shift in the longitudinal direction the most misclassified was the forearm supination (Figure $12 \mathrm{c}$ ).

\subsubsection{Effect of Noise and Reduced Number of Channels in the Test Phase}

In the second part of this study the performances of the Variog feature space were analyzed in two cases. In presence of noisy channels and with a random reduction of the number of channels in the testing phase. In case of noisy channels in the testing phase, the number of channels used in testing and training was the same. The channels with noise were selected randomly for 10 times for each trial and the results were averaged for each subject. The results of this analysis are shown in Figure 14. The first important result of this analysis is that the presence of only one noisy channels drop the classification accuracy of all methods of $\sim 10 \%$ respect to the intact matrix (IM), and continue to decrease progressively increasing the number of noisy channels. The same test has been done for the amputee subject (Figure 15), in this case the IM matrix had 142 electrodes and the Gaussian noise was added in the same way as for the able-bodied subjects. It can be noticed that in this case only one noisy channel caused a degradation of the classification accuracy more evident than for the ablebodied subjects.

In Figure 14 in blue is also represented the case in which there is a progressively reduction of the number of channels in the testing phase for the pattern recognition algorithm with the variogram. The reduction of the channels in the testing phase can be due to the presence of channels with poor quality. Assuming that the detection of poor quality channels for HD EMG is possible, as described in [50], and that it is possible to remove them, it will be possible continue to use the prosthesis without retraining. The classical 


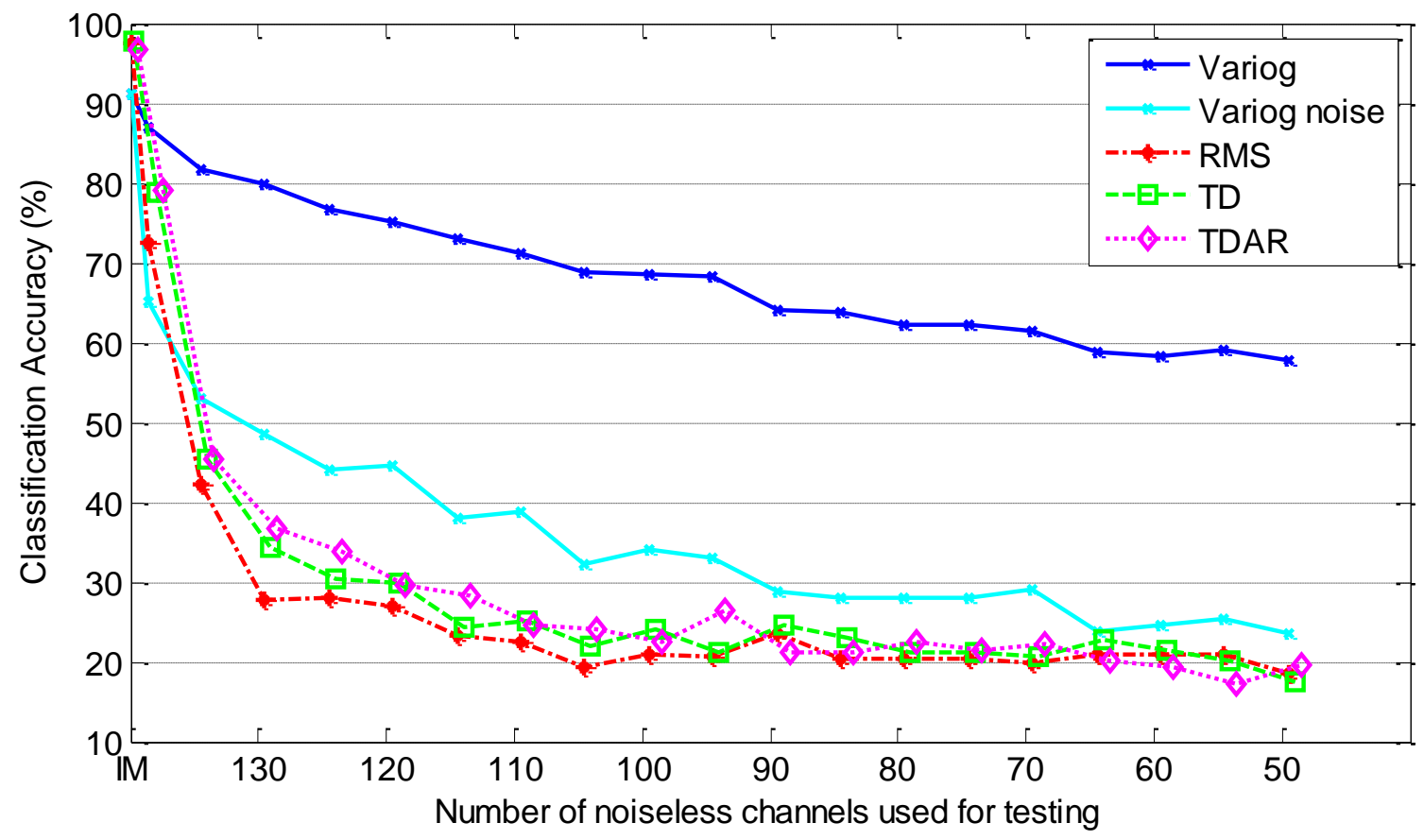

Figure 15. Classification accuracy (7 classes) for the amputee subject as a function of the number of channels used in the classification test for Variog and as a function of the noiseless channel on the other 4 cases (Variog noise, RMS,TD,TDAR). Reused and modified with permission [30] C 2015 IEEE.

classification methods (RMD, TD and TDAR) do not allow to remove channels, since it is required the same number of channels for training and for testing, while for the variagram small variation of the number of measurements used to calculate the experimental variogram do not change the dimension of the feature space used in variogram. This allows to have a different number of channels in the training and in the testing phase. The channels removed were selected randomly for 10 times for each trial and then averaged for each subject. In Figure 14 the variogram case with a reduced number of channels is depicted in blue. The graph shows that the classification accuracy for 9 classes decreased with the removal of channels in the testing phase. However, it remained $\sim 90 \%$ with up to 30 channels removed and decreased to $\sim 80 \%$ with half of the channels removed. In the same way this analysis has been conducted for the amputee (Figure 15). The number of channels, that here starts from 142, was decreased to up 49 channels in the testing phase. The 
classification accuracy for 7 classes in this case dropped from $92 \%$ to $70 \%$, when removing 36 channels.

\subsubsection{Reduced number of channels}

The last part of this study considers the use of a smaller number of channels, to evaluate the performance of the variogram. The number of the channels in the matrix were reduced to 48 first, and after to 24 . In the case of seven able-bodied subject with 48 channels in the grid the classification accuracy normalized, as in the case of 96 channels, resulted $95.8 \pm 2.3$ for no shift, for shift of the columns averaged over SC1 and SC2 $85.9 \pm 0.9$, for shift of the rows averaged over SR1 and SR2 $94.3 \pm 0.2$. In the case 24 channels for the seven ablebodied subjects the classification accuracy resulted $92.6 \pm 3.1$ for the no shift case, $81.1 \pm$ 0.4 for the shift of the columns averaged over SC1 and SC2, 93.7 \pm 0.6 for the shift of the rows averaged over SR1 and SR2. In the case of the amputee with 48 electrodes the accuracy was 81.6 for no shift, 82.1 for the shift of the columns, and 75.6 for the shift of the rows. Finally the amputee with 24 electrodes provided an accuracy of 70.5 with no shift, while for the two shifts was 80.9 and 75.6 respectively for the columns and for the rows. For the other methods (RMS, TD and TDAR) the results were similar to those of the Figure 10. It is possible to conclude that the reduced number of channels did not fully impair the performance of the variogram, but it is advisable to keep the minimum number of pairs for the estimation of the variogram around 50 [47].

\subsection{Conclusions}

In this study a novel pattern recognition algorithm in myocontrol has been proposed, it uses the spatial correlation among HD EMG channels, as a feature minimally sensitive to electrode shifts and to noisy channels. The measurement of spatial correlation, the experimental variogram, has been used as features space for classification. It has been demonstrated that the variogram method reduces the sensitivity to electrodes shifts with respect to the TD, RMS, and TDAR features. Moreover, the variogram features allowed to 
eliminate channels during the test phase without re-training, maintaining a good classification accuracy for a relatively large proportion of omitted channels. These results indicate that the spatial characteristics of HD EMG can be used as more robust features for EMG pattern recognition than the classic time domain features.

This study has been the first that has exploited the spatial correlation of HD EMG as feature in a pattern recognition algorithm. The robustness and reliability in controlling upper limb prostheses have been improved, nevertheless to implement this solution more technological advancements are necessary. The amount of information available requires very powerful microprocessor that have to fit in a prosthesis. Moreover the HD EMG electrodes have to be flexible, to adhere to different shapes of the stump of amputees, they do not have to require particular preparation before the use, and mainly they have to support the long term usage.

More research and technological advancements are envisaged before the spatial correlation of HD EMG will be implemented in commercial products, however a new way has been open and it has been investigated in literature. 


\section{Exploitation of spatial features of HD EMG}

To overcome some of the issues of prosthesis control with pattern recognition algorithms, new directions have been undertaken in the last few years [51]. One of these it has been the exploitation of HD EMG recordings. This has been supported by improvement in the technology of HD EMG electrodes, and by the development of more powerful microprocessors.

Subsequently the publication of the work presented in the previous chapter [30], other works have been done exploiting the HD EMG signals for control of upper limb prostheses. In [52] the authors used a method previously used in electroencephalogram (EEG) studies, the common spatial patterns (CSP) with HD EMG. This method has been used already for control of myoelectric prostheses [53][54], but without considering the shift and with few EMG electrodes. It has been found that this method improved the classification performance in case of shift respect to TD, TDAR, and respect to the Variog in the longitudinal shift configurations.

Other studies related to the estimation of finger movements [55] [56] have also considered the HD EMG signals. In [55] it has been found that HD EMG can increase the robustness, adding redundant information. While in [56] HD EMG has been used to map the activation pattern of the entire extensor digitorum communis. The spatial activation information has been used to localize finger compartments of the extensor digitorum communis.

Spatial distribution of HD EMG has been also exploited to improve the identification of tasks in patients with incomplete cord injury [57]. The results of this study showed the spatial features significantly improved the classification of the tasks. 


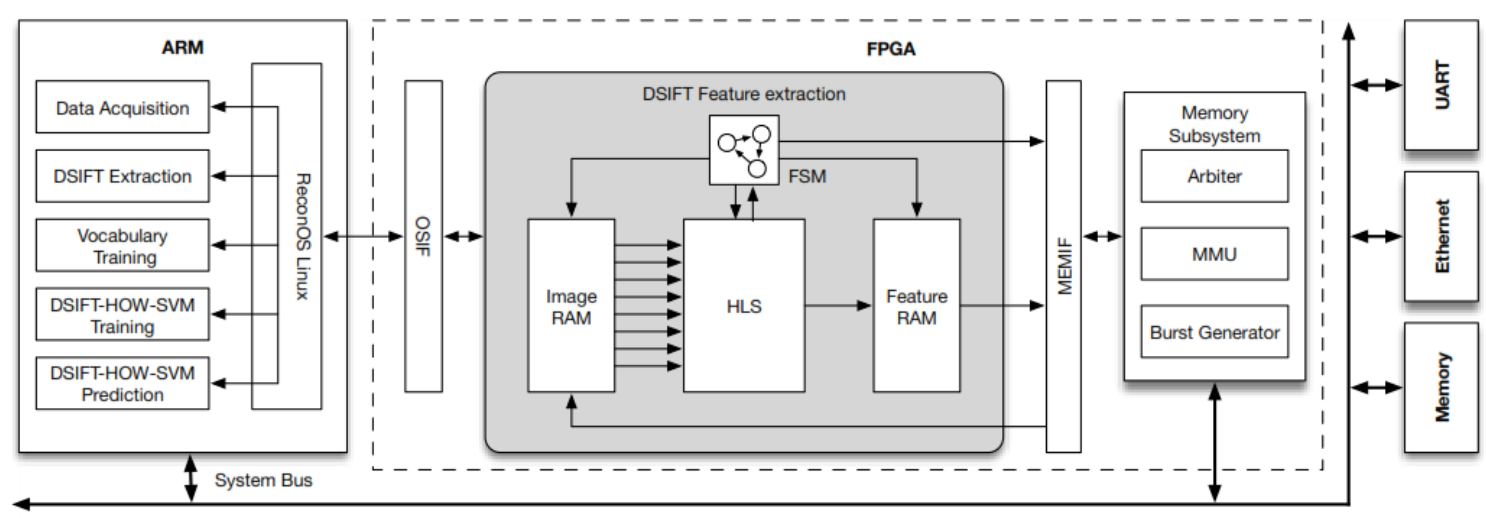

Figure 16. Application architecture of DISFT features extraction hardware thread. From [59].

At the same time some studies have been conducted to develop more powerful microprocessor that can handle a large number of EMG signals. The authors of [58] presented an architecture for embedded HD EMG prosthetics control capable of accelerate up to 4.8 over the software-only solution. In [59] the same authors presented a second architecture for accelerating HD EMG-based control algorithms, where the system achieved a speed-up of 5.5 over the software-only version (Figure 15). This new architecture has been tested with a new pattern recognition method [59][60], based on computer vision features and SVM classification, which results robust to electrodes shift and noisy channels. The approach is based on DSIFT (Dense Scale Invariant Feature Transform) features [61], with fixed orientation, extracted in a dense grid of points from an image. In imagine categorization tasks the DSIFT features are extracted at multiple scales and combined with histogram-of-visual-words (HOW) [62], forming scale invariant features [63]. In [59] the DSIFT-HOW features have been extracted from the 2D image representation of HD EMG signals (192 channels). These features have been used form movement classification showing better classification accuracy in presence of noisy channels respect to the TD features. 
The increasing number of studies using HD EMG, and in particular in the field of classification of movement, demonstrate that they can represent a valuable means to improve the control in upper limb prostheses. They can potentially reduce the drawbacks of the electrodes shift and of the noisy channels, when coupled with more powerful microprocessors. Nevertheless, to be effectively exploited in the prostheses, improvements in the technologies are still needed. They have to be suitable for long term use, this means that they cannot be deployed with the conductive gel, as it has been done in all the work presented previously. It is desirable that for example HD textile EMG will be further developed, so that they can be easily embedded in the socket of the prosthesis. Moreover, it has to be a cheaper technology, to decrease the costs of the modern upper limb prostheses. 


\title{
Part II: The invasive
}

\author{
approach
}




\section{State of the art in implanted EMG sensors for}

\section{myocontrol}

The recently improvements of the technologies for implanted devices, and the need to overcome the drawbacks due to the use of surface EMG electrodes in the control of upper limb prostheses with pattern recognition algorithms, has brought to the development of implanted EMG electrodes. For implanted EMG electrode it is intended an invasive device, permanently positioned into the muscle, which can provide localized measurements [64]. Needles or hook-wire electrodes are usually used for intramuscular EMG recordings, but they are not suitable for long-term use, because the presence of transcutaneous wires, which can cause infections.

Implanted EMG present some advantages respect to the surface EMG, it can measure the activity of deep muscles, achieve better signal-to-noise ratio and do not have the issue of the shift [26]. The actual implanted EMG electrodes are divided in extra and intra-muscular [64]. The extra muscular electrodes are sutured onto the epimysium of the target muscle, while the intra-muscular are inserted directly into the muscle.

Epimysial EMG systems have been proposed with the intent to use them for upper limb prostheses [65] [66] [67]. In [65][66] the epimysial electrodes are connected by wires to a central unit, which send the EMG signals to the prosthesis using radio frequency transmission. Both epimysial electrodes and central unit have been implanted only in animals and just to record signals from the limbs, they are not yet implemented in a real prosthesis. In [66] some problems with the contacts between electrodes and the circuit board were encountered, with the loss of the EMG signals. In another study [67] has been proposed a wireless implantable EMG system for control of powered prostheses. The implantable system proposed consists of two epimysial electrodes that can transmit wireless EMG signals to an external receiver. It uses a transmission protocol tailored for the purposed. The power transfer is done by an external coil and the RF link is based on the standard IEEE 802.15.4 [68]. Also in this case it has been tested only in animal to record 


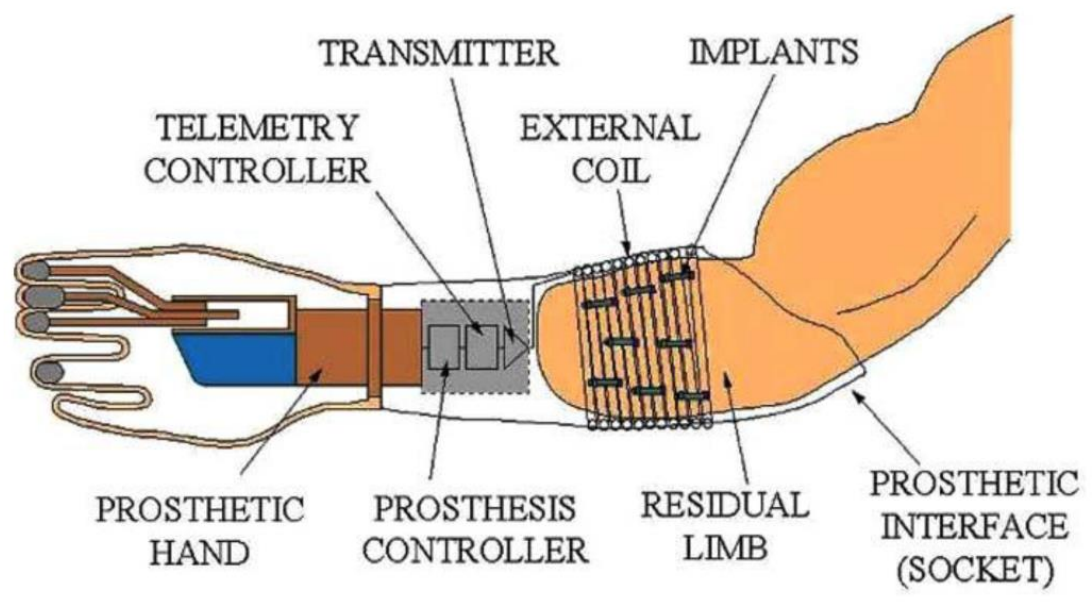

Figure 17. Overview of the system IMES. Reused with permission from [69]

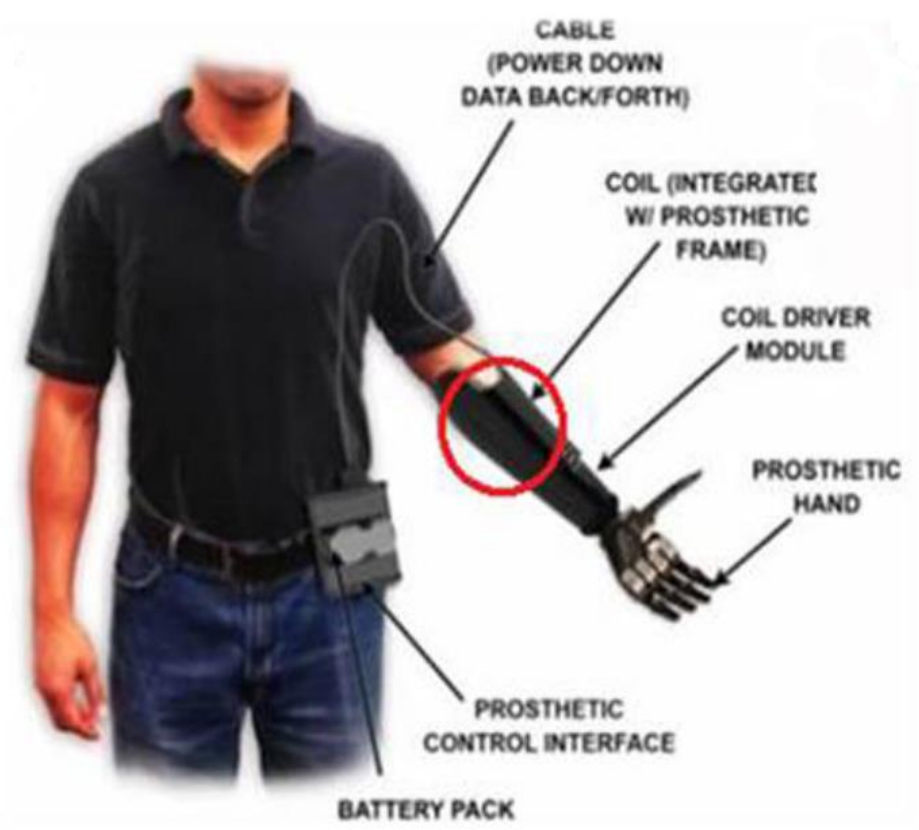

Figure 18. IMES system, with permission from [70]

EMG signals, moreover the implantable electrics are kept outside the body of the animal. Few other studies exist on this topic which use intra-muscular implanted. The most advanced and detailed study has been done by Weir [69], which proposed a multichannel EMG sensor system that can record, process and transmit wireless EMG signals. In Figure 17 
the system is illustrated, it comprises the implanted devices, the external powering coil, telemetry and prosthesis controller, and the prosthesis hand. The large external coil has to be placed around the limb. The implantable myoelectric sensors are called IMES. They can record EMG signals and transmit them wirelessly via an electro-magnetic coil built in to the socket of the prosthesis. This system has started the clinical trials on one amputee subject (Figure 18) [70], but only preliminary results are available. It seems that the system allowed a more intuitive control of the three DoF provided by the prosthesis, but there are still many aspects that have to be improved. In fact, there are constraints in the placements of the EMG sensors, since they have to be completely enclosed in the area covered by the external coil and parallel, making this kind of sensors suitable only for amputees with a round stump, as in transradial or transhumeral amputees [65]. The IMES system presents also practical difficulties for the overall efficiency due to the usage of a large inductive power fields.

Considering the studies that have been conducted to control the prosthesis with implanted EMG and the increasing interest in this field, it is possible to assert that the use of implanted EMG sensors can potentially solve the issues coming from the use of sEMG along with implanted nerve electrodes for sensory feedback [71].

The technology for such purpose has to be designed taking into account the application and the particular environment in which will be used. The system has to be designed in a way that can be applied to any type of amputees, with a specific standard communication protocol.

In this second part of the thesis it has been proposed a new system for controlling upper limb prosthesis with wireless implanted EMG. It has been proposed a system that uses the RF link for communication between implants and controller.

A systematic study has been done to investigate the use of a RF wireless link based on a specific standard for implanted communications. The study started with the modeling of the wireless link, considering that the means of propagation, in this case, is the human body. In order to determine the feasibility of the system proposed, the propagation losses of the link 
have been investigated, along with an evaluation of the specific absorption rate and a preliminary link budget analysis. 


\section{Background of wireless RF implanted devices}

Recently applications of implanted devices are monitoring and diagnosing cardiac pathologies, cancer, asthma and neurological disorders [72], moreover also people with disabilities can be helped by cochlear and retina implants and limb prostheses [73]. The development of these new applications has determined the standardization of networks formed by devices that are placed around, on the body, or implanted inside the human body. Such networks are called body area networks (WBANs) and they are regulated by an international standard, IEEE 802.15.6 [74]. Implanted devices are known form many years, the firs implantation of a pacemaker into a human was in 1958 [75], but only recently there have been the need of communication with the implant and retrieve information from it. Before the standardization of the WBAN the communication with the implants was done mainly over an inductive link. In the inductive link the transfer of data is done with two coils, one external and one on the implant. This type of communication does not allow high bit rate and the range is quite low (limited to the "touch" range), this means that for deep implants is impractical [75]. Moreover, the external coil must be positioned accurately to get a reliable link. For this reasons the RF implanted devices are replacing the inductive communication [76]. The RF transmission inside the human body is complex and has to be completely characterized. Implanted wireless RF device have built-in antennas that can transmit and receive signals and they operate inside the human body. They have to be very small, require very low power and be efficient. In the following paragraphs the main aspects of implants communications are summarized.

\subsection{Medical Implant Communication System (MICS) band}

Before the issue of the IEEE 802.15.6 standard, the European Telecommunication Standards Institute (ETSI) and the Federal Communication Commission (FCC) have standardized the Medical Implant Communication System (MICS) [77][78]. This standard is specific for implanted communication from inside the body (in-body) to outside the body (on-body or off-body) or from in-body to in-body, i.e. between medical implants. For these 
communications a frequency band is allocated, from 402 to $405 \mathrm{MHz}$ with a maximum bandwidth of $300 \mathrm{kHz}$. The power limit is fixed to $25 \mu \mathrm{W}$ on the surface of the body. The frequency band allocated for MICS is recognized almost worldwide and it is shared only with the meteorological balloons $(400-406 \mathrm{MHz})$. This assure no interference with other radio frequency bands. This frequency band has been also included in the IEEE 802.15.6 standard for implanted applications. The antennas designed for this frequency band can be small enough to be fitted inside the human body and have also good performance over two meters transmission range. Small antennas are efficient radiators in the MICS frequency band considering the characteristics of the human tissues in such frequencies.

\subsection{Radio Frequency and Human Body}

For wireless implanted devices the means of propagation is the human body. The human body is not an ideal medium, it is partially conductive and is composed by materials with different dielectric constants, which can vary with the frequency of operation. To model implanted communication is extremely important understand the effect of the human body on the RF signals. The tissues and organs composing the human body are characterized by own conductivity $(\sigma)$, dielectric constant $(\varepsilon)$, and penetration depth $(\delta)$. These electrical properties have to be known for the frequency of interest, to characterize the human body as a medium. In Table 1 are summarized the electrical properties for the main tissues forming the body, muscle, fat, and skin at center of MICS frequency band.

Table 1. Dielectric properties for human tissues at $403.5 \mathrm{MHz}$

\begin{tabular}{|l|l|l|l|}
\hline $\begin{array}{l}\text { Human } \\
\text { tissue }\end{array}$ & $\begin{array}{l}\text { conductivity }(\boldsymbol{\sigma}) \\
{[\mathbf{S} / \mathrm{m}]}\end{array}$ & dielectric constant $(\boldsymbol{\varepsilon})$ & $\begin{array}{l}\text { Penetration depth }(\boldsymbol{\delta}) \\
{[\mathrm{m}]}\end{array}$ \\
\hline muscle & 0.7972 & 57.1 & 0.0525 \\
\hline fat & 0.0411 & 5.5783 & 0.3085 \\
\hline skin & 0.6895 & 46.7060 & 0.0551 \\
\hline
\end{tabular}


The dielectric constant describes how the material is affected by electric fields and varies with the frequency. The conductivity describes how much the electromagnetic wave is attenuated when transit the body tissues, it varies with the frequency. The penetration depth is the depth at which the electric field has been attenuated of a certain factor. In the human body the penetration depth can be described by the following expression:

$$
\delta=\frac{1}{\operatorname{Re}[\gamma]}
$$

where $\gamma$ is the propagation constant. Also the penetration depth varies with frequency.

\subsection{Implanted antennas}

The implanted antennas operate in a lossy environment, the human body. The wave propagation velocity inside the human body is lower compared to the free space, and also the wave length results shorter. At $403.5 \mathrm{MHz}$, center of the MICS band, the wave length in free space is $74 \mathrm{~cm}$, while in the human body is $9 \mathrm{~cm}$. This imply that implanted antennas have dimensions less than 10 percent of the wave length in free space, therefore the transmission efficiency is very low. Nevertheless, a small antenna in the MICS band is an efficient radiator due to the characteristics of the human tissues [76]. The implanted antennas can be electrical or magnetic. The electrical antennas usually generate large component of the electric field (E-field) normal to the tissues interface, overheating the fat tissue. The magnetic antennas, on the contrary generate an E-field that is mainly tangential to the tissues, that does not overheat the fat. In term of radiation performance and safety for the humans the magnetic antennas would be a better candidate for implanted devices [76]. The shape of the implanted antennas it is determined by the applications, i.e., it depends of the place where it has to be placed. 


\subsection{Safety issues}

The exposure to high intensity of RF radiation can cause a heating effect of human tissues and this can be harmful if the temperature increases 1 degree Celsius. A parameter used to determine if a wireless device it is not harmful for the human beings is the specific absorption rate (SAR). It quantifies the RF energy absorbed in the human tissues. The relationship between radiation and SAR is given by:

$$
S A R=\frac{\sigma|E|^{2}}{\rho} \quad[W / K g]
$$

where $\sigma$ is the electrical conductivity of the tissue $(\mathrm{S} / \mathrm{m}), \mathrm{E}$ is the induced electric field strength $(\mathrm{V} / \mathrm{m})$, and $\rho$ is the density of the tissue $\left(\mathrm{Kg} / \mathrm{m}^{3}\right)$. SAR is a measure of the amount of heat in the antenna surrounding. This parameter is extremely important for the human health, therefore there are limits and regulations that have been imposed by authorities. These limits are summarized in the Table 2.

Table 2. SAR limits.

\begin{tabular}{|c|c|c|c|}
\hline Region/Country & Reference to SAR limits & Limits (W/Kg) & \\
\hline \multirow[t]{3}{*}{ Europe } & \multirow{3}{*}{$\begin{array}{l}\text { ICNIRP Guidelines } 1998 \\
\text { (ICNIRP 1998) } \\
\text { over } 10 \text { gr of tissue }\end{array}$} & whole body & 0.4 \\
\hline & & head and trunk & 2 \\
\hline & & limbs & 4 \\
\hline \multirow[t]{3}{*}{ US } & \multirow{3}{*}{$\begin{array}{l}\text { American Standard ANSI C95.1 } \\
\text { (ANSI 1992) } \\
\text { over 1gr of tissue }\end{array}$} & whole body & 0.08 \\
\hline & & Head and trunk & 1.6 \\
\hline & & $\begin{array}{l}\text { Extremities (hands, } \\
\text { wrists, feet, and ankles) }\end{array}$ & 4 \\
\hline
\end{tabular}




\begin{tabular}{|l|l|l|}
\hline Australia & $\begin{array}{l}\text { Australian Standard } \\
\text { AS/NZS 2772.1 }\end{array}$ & ICNIRP guideline \\
\hline Japan & $\begin{array}{l}\text { STD-T56 } \\
\text { (ARIB 1998) }\end{array}$ & ICNIRP guideline \\
\hline
\end{tabular}

In the Table 2 are shown the limitations and regulations divided per region in the world. The guidelines provided by ICNIRP (International Commission on Non-Ionizing Radiation Protection) [79] are followed in Europe and several countries in the world, such as Japan and Korea. These guidelines states that the local SAR, averaged over a cube of $10 \mathrm{~g}$ of tissues, should not exceed $2 \mathrm{~W} / \mathrm{Kg}$ for head and trunk and $4 \mathrm{~W} / \mathrm{Kg}$ for limbs. The SAR limits for the human limbs are usually lower since in arms and legs the circulatory system acts as a coolant. The ICNIRP guidelines have been accepted also by the International Telecommunications Union (ITU) [80] and by the World Health Organization (WHO) [81]. The SAR limit for head and trunk in US has been fixed by FCC and it is $1.6 \mathrm{~W} / \mathrm{kg}$ averaged over $1 \mathrm{~g}$ of tissue [82]. Despite the increasing spread of implanted devices, there have not been issued specific regulations concerning the SAR. It has been assumed that the current limits for electromagnetic exposure are valid also in case of implanted wireless devices.

\subsection{Channel modeling}

The human body is a heterogeneous propagation environment characterized by layers of different type of tissues with a different thickness and each with a dielectric property. The RF signals characteristics change when transmitted. The changes depend on the environment and on the distance between the transmitter and the receiver. The profile of the transmitted signal can be obtained by the received signal and it is called channel model. In the case considered the medium of propagation is the human body, which is a lossy heterogeneous medium with high permittivity. To guarantee low latency and high reliability 
a good communication link is needed. To establish a good communication link the first step is a good channel model.

Channel models are often achieved by physical measurements of the path loss in different experimental environment. For practical and ethical reasons, in the case of implanted devices, the measurements to obtain the channel model cannot be done by human trials, but are done by simulations. Many propagation models describe the conditions in the air (or free space) taking into account the losses due to fading. In case of body area networks, the propagation path can also be subject to fading due to shadowing by body posture, reflection, diffraction or energy absorption. The path loss depends on distance and frequency and in case of body area networks it can be represented in terms of distance $d$, as [83]:

$$
P L(d)=\frac{G_{R} P_{T}}{P_{R}(d)}
$$

where $P_{T}$ is the transmitted power, $P_{R}$ is the received power, and $G_{R}$ is the gain of the receiving antenna. The transmitting antenna in MICS band is considered to be part of the channel [84] [75]. The path loss can be statistically modelled in dB with the following formula, based on the Friis formula [84] [85] [86]:

$$
P L_{d B}(d)=P L_{0}+10 * n * \log _{10} \frac{d}{d_{0}}
$$

where $P L_{0}$ is the path loss in $\mathrm{dB}$ at a reference distance $d_{0}$ expressed in $\mathrm{mm}, d[\mathrm{~mm}]$ is the antenna separation, and $n$ is the path loss exponent, which depends on the environment where the RF signal propagates. In free space $n=2$. This equation does not take into account the shadowing component due to different body tissues and antenna gain in different directions. These losses are considered adding a term $\mathrm{S}$, wich is a normal distribution

$$
P L_{d B}(d)=P L_{0}+10 * n * \log _{10} \frac{d}{d_{0}}+S
$$


The shadowing component $S \sim N\left(0, \sigma_{S}^{2}\right)$ takes in to account that there are different values of the path loss for same distances between transmitter and receiver.

\subsection{Finite Difference Time Domain (FDTD) simulations}

There are several numerical modeling techniques for analyzing electromagnetic problems in the field of body wireless communication. The most used method is the Finite-Difference Time-Domain (FDTD). The FDTD method requires the division of electromagnetics structures into small cells, and it is suitable for modeling inhomogeneous media and complicated boundaries [75]. The FDTD is a technique to solve Maxwell's equations. It employs finite differences as approximations to both the spatial and temporal derivatives that appear in Maxwell's equations. Yee has proposed this method in 1966 [87], from then many extension and improvements have been published. This method has been used to solve a number of problems in electromagnetics. This method requires a large amount of memory, indeed it is required to mesh the entire computational domain, and the cells need to be small compared to the wavelength. Even if this method is computational expensive and requires a large amount of memory, it is the most used in the electromagnetics simulators.

\subsection{Human model and electromagnetic simulation tool}

The investigation of propagation losses for implanted devices in human body cannot be done with in-vivo measurements. They can be done with phantoms filled with a fluid that simulate the human tissue or by numerical simulations. The latter allow considering multiple tissues that compose the human body. In this study the numerical simulation has been preferred. The human body model used in this work has been obtained by magnetic resonance images (MRI) of healthy volunteers and is provided by SPEAG [88]. The model represents a man of 34 years old (denoted Duke) and it is part of the Virtual Family, which has four anatomical models (two adults and two children). These models include 80 different body tissues with dielectric properties based on the database generated by Gabriel [89]. The maximum grid step of the human model is used is $2 \mathrm{~mm}$. The electromagnetic 
simulation tool adopted in this work is SEMCAD X [90], it is a 3D solver which carried out simulations with the FDTD method. 


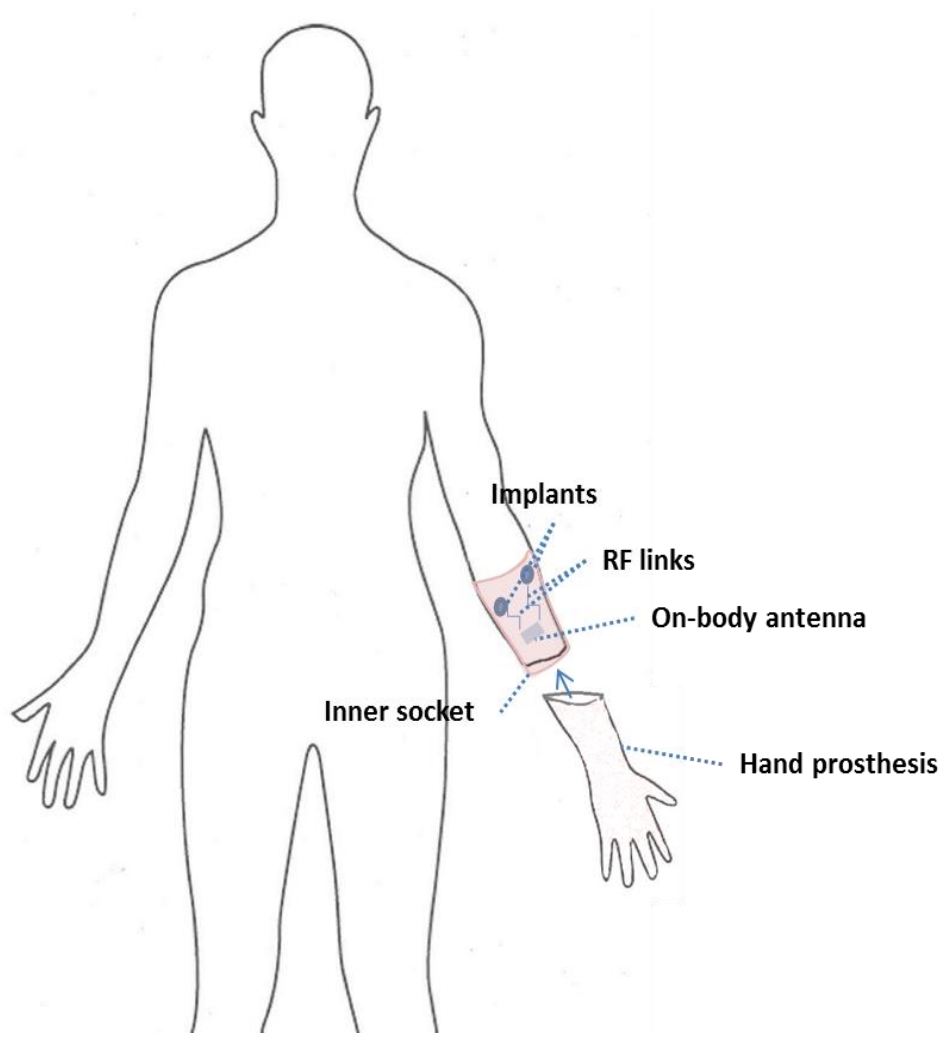

Figure 19. System overview. Two implanted EMG sensors are implanted in the forearm of the amputee. The sensors record and process the EMG signals, which are transmitted to an external antenna placed on the socket prosthesis. The signals are then used to drive the hand prosthesis. Reused and modified with permission [73] @ 2015 IEEE.

\section{Characterization of In-Body to On-Body wireless Radio Frequency link for upper limb prostheses}

In the last few years the research in the field of upper limb prostheses has considered the possibility to utilize implanted devices that can record and transmit wirelessly EMG signals. This solution can potentially provide robustness and reliability to prostheses, overcoming the drawbacks raised by the use of surface EMG electrodes. Nevertheless, the systems that have been proposed in literature do not provide a solution that can be easily adopted and implemented. Moreover they cannot be exploit for each type of amputee. 

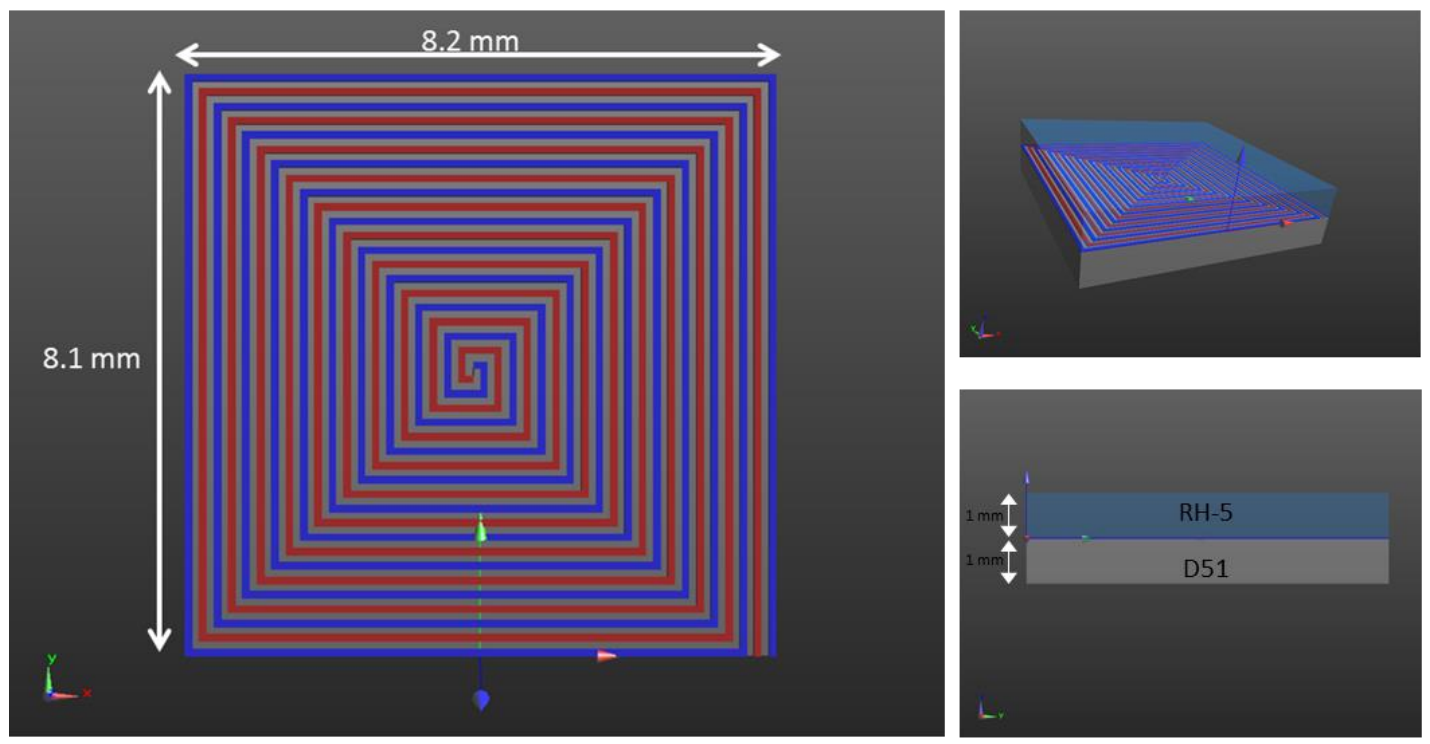

Figure 20. Implanted antenna

In the following will be described a new approach that has been published in [91] [92] by me as first author. Text and results reproduced from these papers are not cited explicitly in the following. The objective of this study was to provide information, as the channel model, the SAR level and the link budget, on the feasibility of wireless implanted EMG sensors.

The system proposed is represented in Figure 19. It consists of two devices implanted in the forearm of an amputee. These devices can record and process EMG signals, which are transmitted to an external antenna placed on the socket prosthesis and then used to control the robotic hand. The wireless link is based on IEEE 802.15.6 standard [74] and operates in the MICS band. The system can be considered as a WBAN, with two implanted nodes and one on-body. The first step to design and realize such a system is the characterization of the wireless link. The characterization consists in the channel modeling and SAR measurements. For simplicity the system considered has two implanted antennas and one on-body antenna, but the same considerations and results are valid with more implanted devices. 


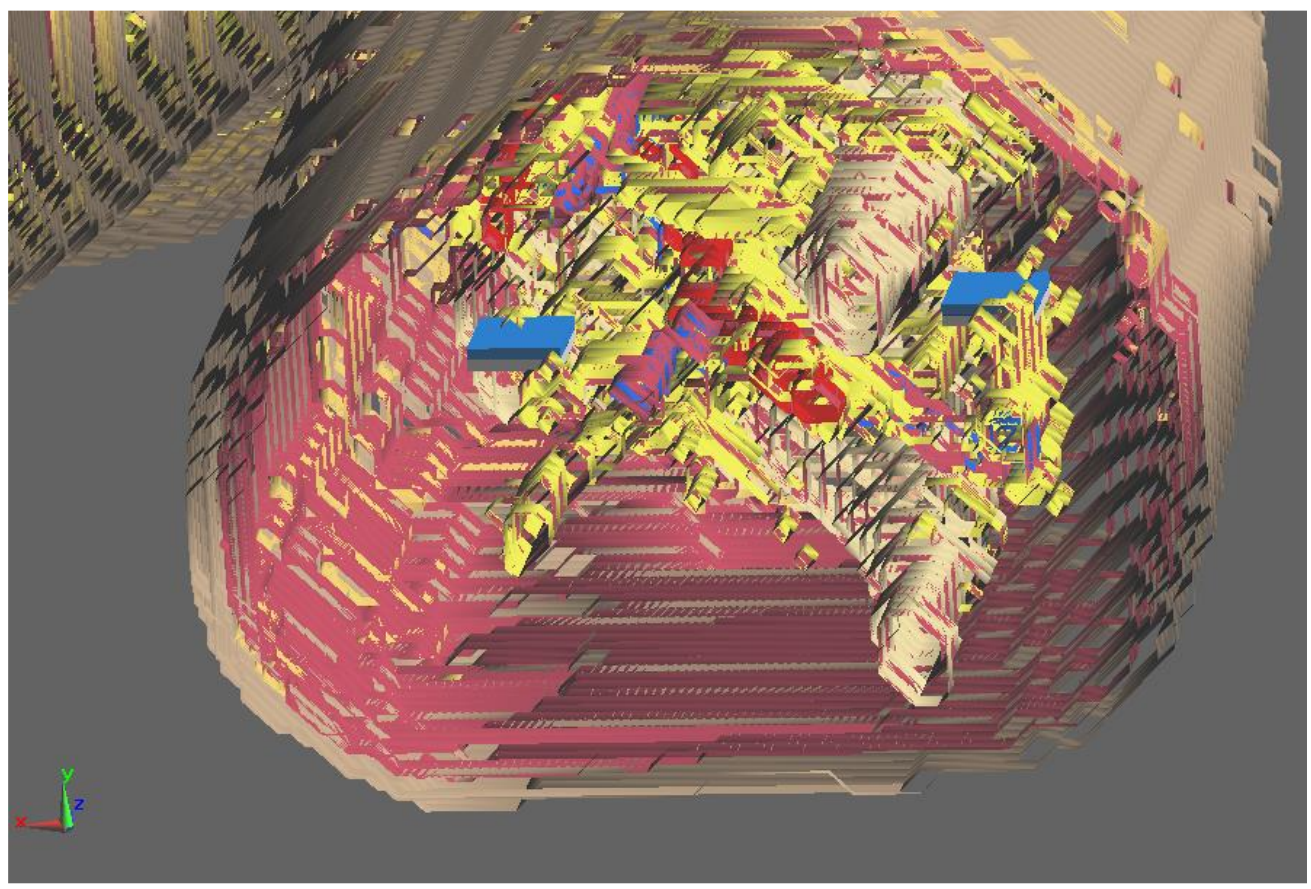

Figure 21. Section of human arm with two implanted antenna. Reused with permission from [92].

\subsection{Implanted antennas}

The implanted antenna selected has been used also by IEEE802.15.TG6 committee to recommend channel models [83]. However the same committee has specified that the channel models described in [83] do not provide absolute performance and that each application requires a specific channel model. The shape and dimension of the antenna described in [83] can fit in a small device that has to be implanted in a forearm, or in the shoulder, depending on the type of amputation. In the system considered (Figure 19) the antennas have been implanted in the forearm.

The antenna is composed of a single metallic layer of copper. The metallic layer is printed on a side of a D51 (NTK) substrate with dielectric constant $\varepsilon_{r}=30$, loss tangent $\tan \theta=$ 0.000038 , thickness of $1 \mathrm{~mm}$, and covered by RH-5 substrate with dielectric constant $\varepsilon_{\mathrm{r}}=$ 1.0006, loss tangent $\tan \theta=0$ and thickness of $1 \mathrm{~mm}$ (Figure 20). 


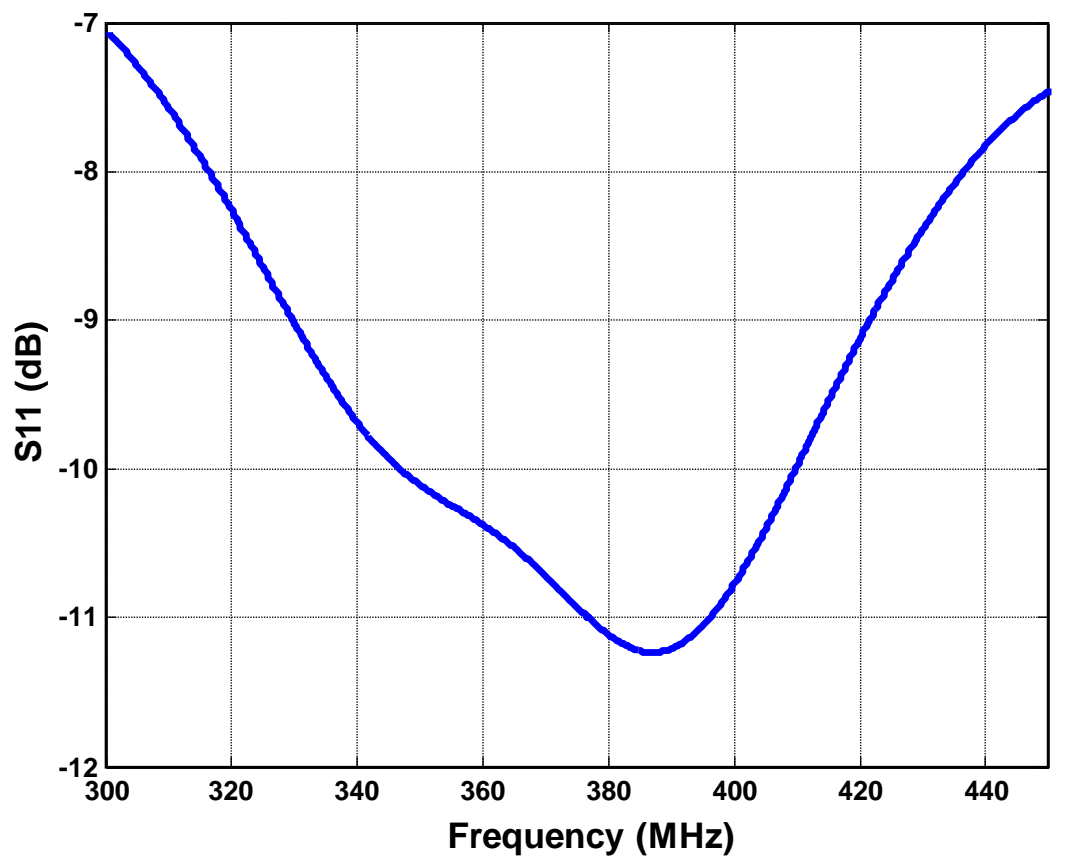

Figure 22. Return loss of the implanted antenna. Reused with permission from [92].

The antenna has been designed to operate inside the human body at the MICS frequency band. To proceed with the simulations, the two antennas, as in described above, have been implanted in the forearm of the 3D human model. They have been positioned approximately in the wrist extensor and flexor muscles. In Figure 21 it is shown a section of the arm with the implants.

The reflection coefficient has been calculated to verify that the implanted antenna operates in the frequency range of the MICS band. The return loss has been simulated fixing the central frequency to $403.5 \mathrm{MHz}$ with the FDTD simulation platform SEMCAD X. It has been found that $S_{11}$ value is about $-10 \mathrm{~dB}$ at $403.5 \mathrm{MHz}$ (Figure 22), which confirms a good impedance match.

In the Figure 23 is shown the 3D gain polar plot. The gain varies with the direction and is not isotropic. The maximum gain, taking into account also the losses of the body phantom, is $-55.37 \mathrm{dBi}$. The maximum directivity is observed in the direction opposite to the $\mathrm{x}$ axis (in 


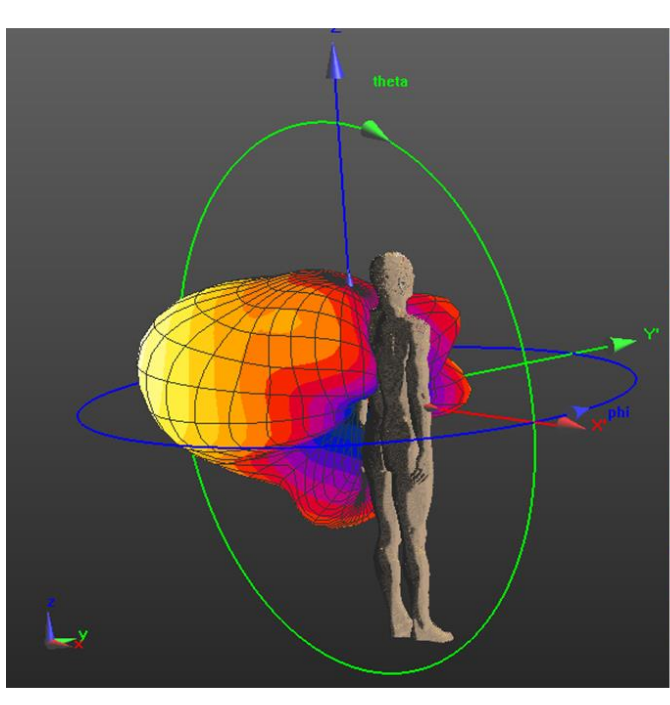

(a)

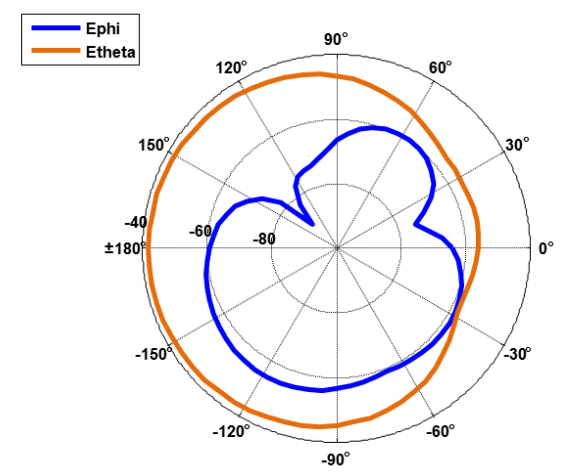

Plane $X Y$

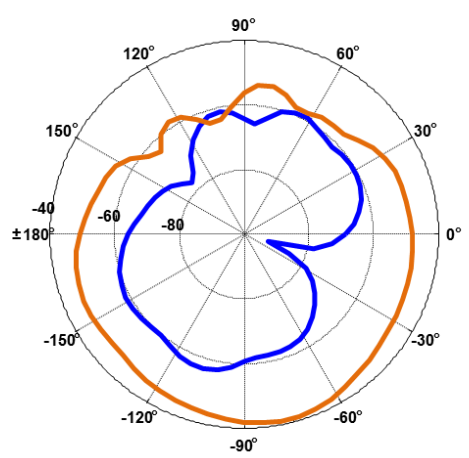

Plane $X Z$

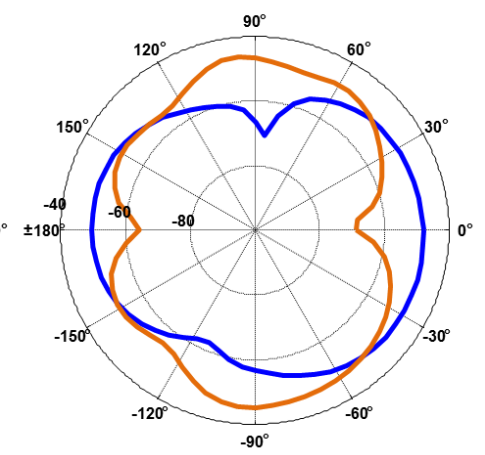

Plane YZ

(b)

Figure 23. 3D Gain polar and radiation pattern plots of the implanted antenna (a) 3D gain. (b) values of $E_{\phi}(d B)$ and $E_{\theta}(d B)$ in three different planes. The coordinate system is shown in figure. Reused with permission from [92].

the $X Z$ plane), but it is possible to observe (Figure 23b) that the $E_{\theta}$ and $E_{\varphi}$ components are similar. 


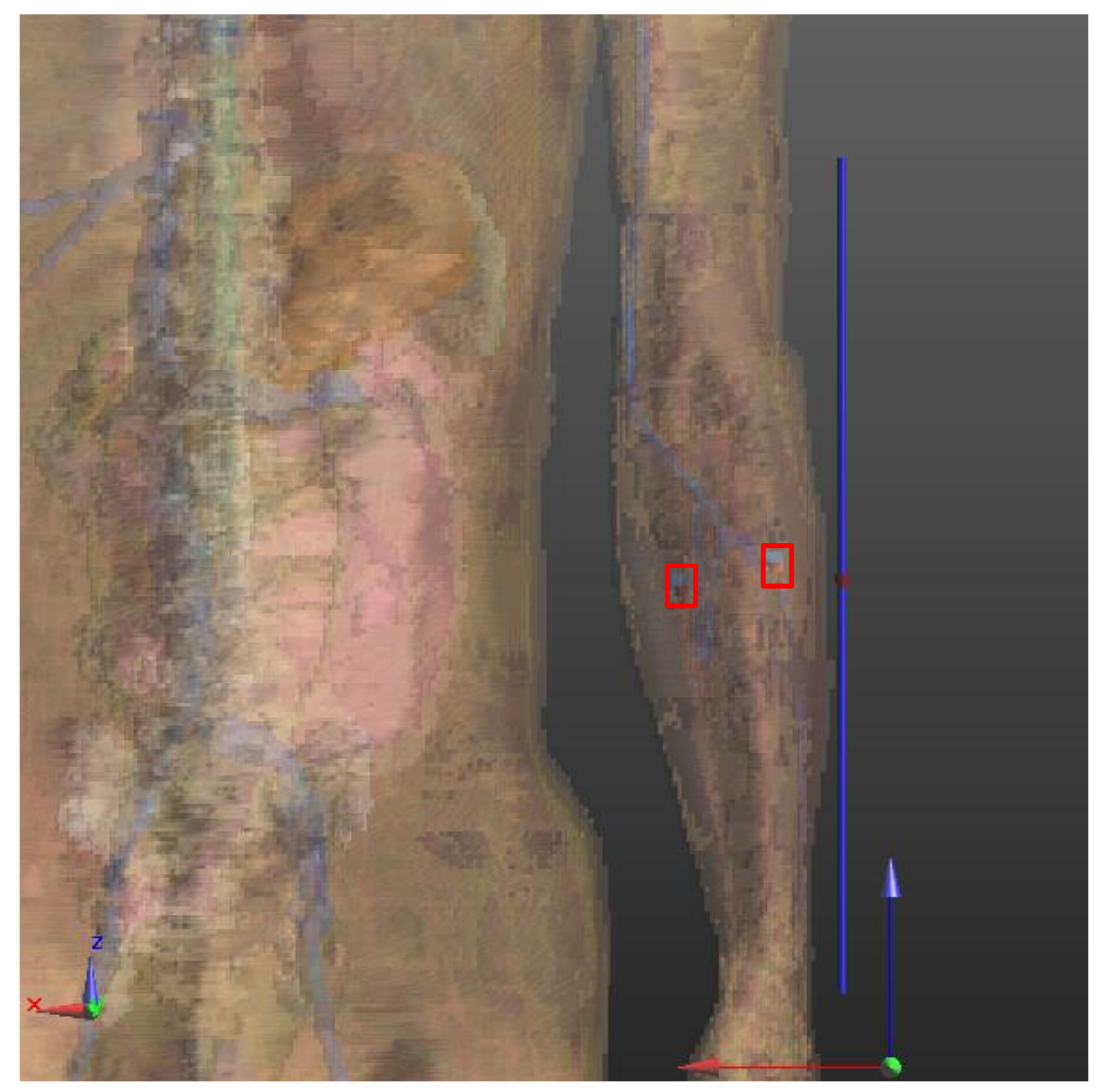

Figure 24. Half-wave dipole antenna near the arm of the 3D human model. Reused with permission from [92].

\subsection{On-body antennas}

The antenna that positioned in the socket of the prosthesis is called the external antenna or on-body antenna. In the first stage of the analysis conducted to design the new system proposed in this study, it has been selected, as on-body antenna, a half wave dipole. This type of antenna has simplified the analysis and reduced the simulation time.

As a refinement a more realistic antenna has been designed and investigated in a second stage of the study, it will be described below.

The half-wave dipole has been designed considering that the wavelength in free space is $\sim 74 \mathrm{~cm}$, so the length of each arm has been set to $180 \mathrm{~mm}$. The thickness has been fixed to $2 \mathrm{~mm}$ and the gap between the arms to $1 \mathrm{~mm}$. The antenna has been positioned first in the 


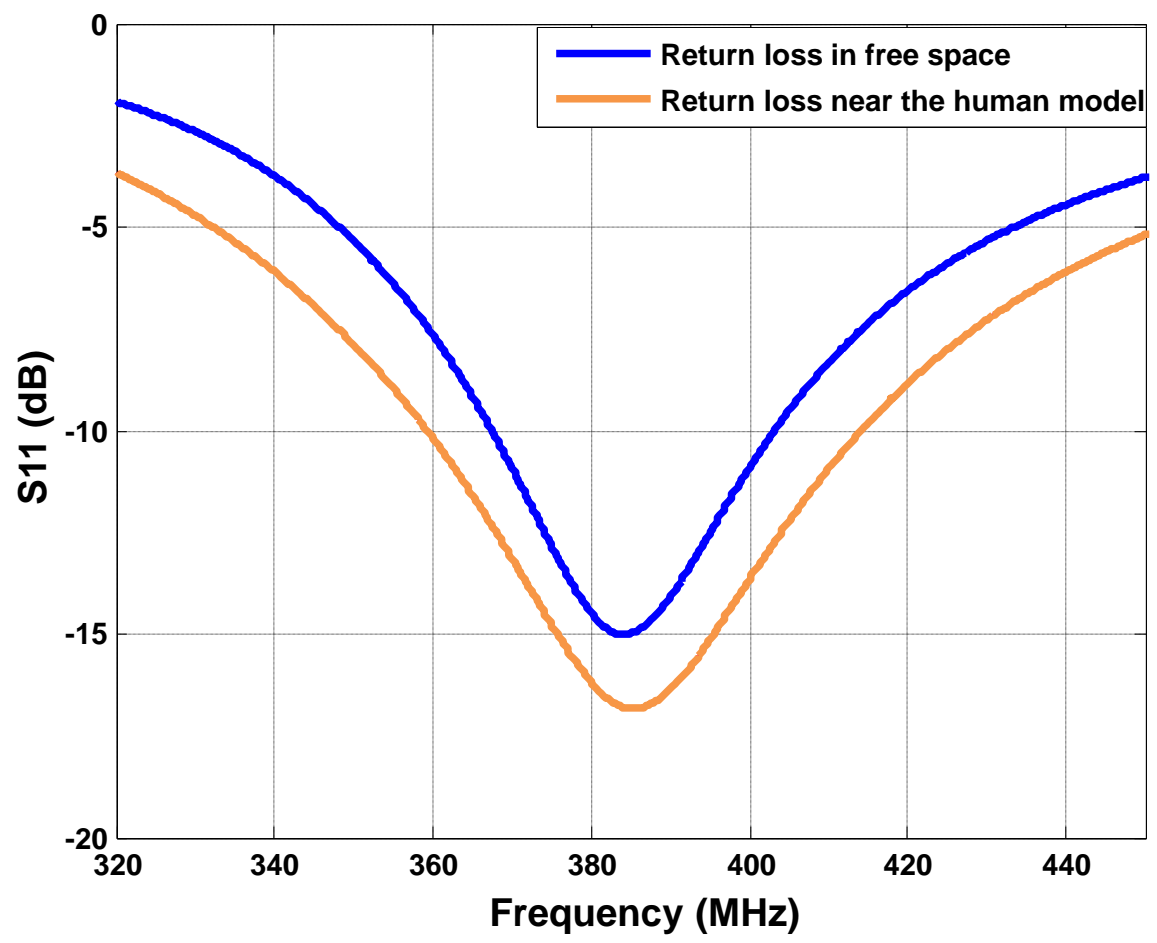

Figure 25. Return loss of the half-wave dipole antenna. Reused with permission from [92].

free space and after near the human body at $1 \mathrm{~cm}$ from the arm in the near field region (as in Figure 24), in both cases the return loss has been calculated (Figure 25). The $S_{11}$ values are similar showing a good impedance matching in the MICS band $\left(\mathrm{S}_{11}\right.$ at $403.5 \mathrm{MHz}$ is $\sim-10$ $\mathrm{dB}$ in free space and $\sim-12 \mathrm{~dB}$ near the human model).

The gain and the values of the $E_{\phi}$ and $E_{\theta}$ components are showed in Figure 26 . The maximum value of the gain is $-3.2 \mathrm{dBi}$ (Figure 26a). $E_{\phi}$ and $E_{\theta}$ components have smaller values in the directions of the human body as can be noticed in Figure $26 \mathrm{~b}$.

This antenna has been very helpful to analyze the electromagnetic characteristics of the system proposed, but it is not a practical solution, when considering upper limb prosthesis. It cannot be embedded in the socket of the prosthesis. For this motivation a different antenna has been designed, a helical dipole antenna [93], with constant radius and feed at the midpoint. 


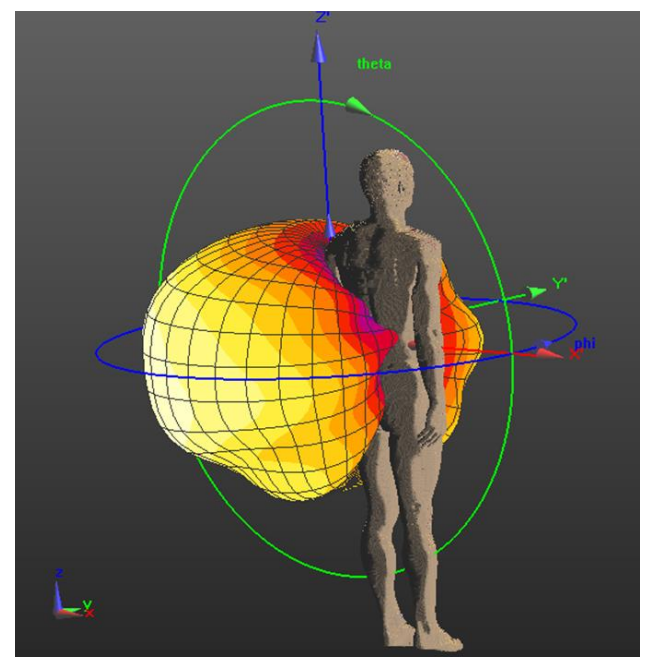

(a)

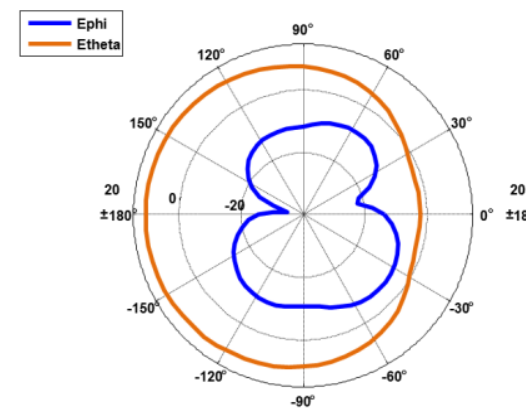

Plane XY

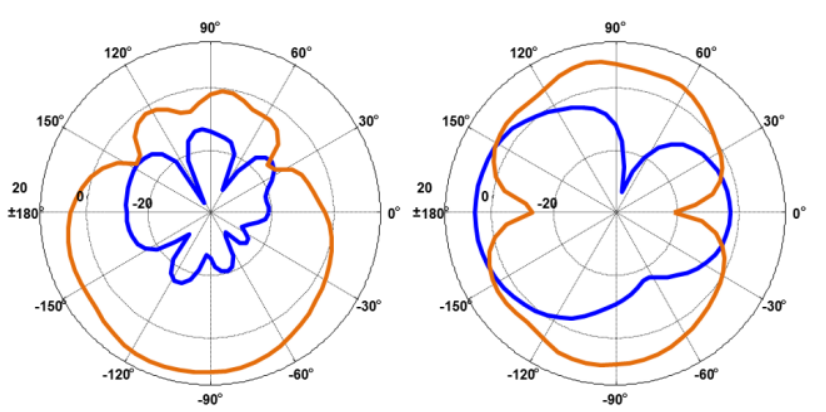

Plane XZ
Plane YZ

(b)

Figure 26. 3D Gain polar and radiation pattern plots of the dipole antenna (a) 3D gain. (b) Values of $E_{\phi}(d B)$ and $E_{\theta}(d B)$ in three different planes. The coordinate system is shown in figure. Reused with permission from [92].

The helical antenna has been designed to operate in the MICS frequency band when positioned near the human body and optimized with the FDTD simulator SEMCAD. The conductor wire has a radius of $1.433 \mathrm{~mm}$, the distance between the turn has been fixed to $180 \mathrm{~mm}$, and the total height of the antenna is $152.4 \mathrm{~mm}$ (Figure 27a). The diameter of the helical is $101 \mathrm{~mm}$, which allow the antenna to be positioned around the arm (in the socket prosthesis), as in Figure 27b. 


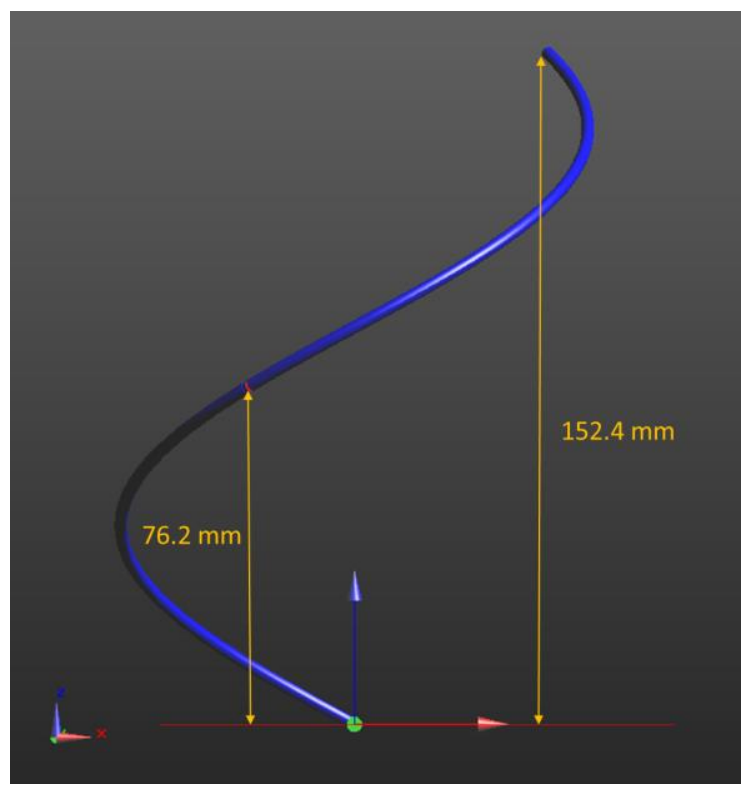

(a)

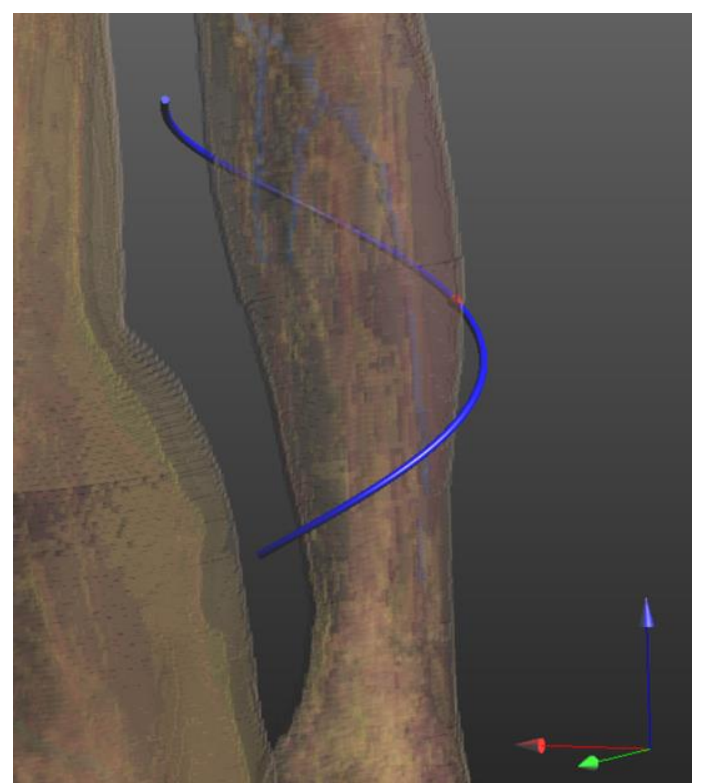

(b)

Figure 27. Helical dipole antenna. (a) Dimensions. (b) position of the helical antenna near the human body. Reused with permission from [92].

To verify the frequency of operation the return loss has been simulated in the free space and in near the 3D human body (as in Figure 27b). There is a clear de-tuning of the antenna when positioned in free space Indeed $S_{11}$ is $\sim-3 \mathrm{~dB}$ in free space, (Figure 28), while the $S_{11}$ value at $403.5 \mathrm{MHz}$ is $\sim-11 \mathrm{~dB}$ when the antenna is around the arm of the human body (orange curve). The gain and radiation pattern are similar to the half-wave dipole as shown in Figure 29a and Figure 29b. The maximum gain is $-7.06 \mathrm{dBi}$.

\subsection{Channel model for upper limb prostheses}

The path loss has been investigated with the 3D solver SEMCAD-X [90] by FDTD simulations. The path loss is defined in terms of the transmission coefficient $\left(-\left|S_{21}\right|_{\mathrm{dB}}\right)$ with respect to $50 \Omega$ as the ratio of the input power at port $1\left(\mathrm{P}_{\text {in }}\right)$ to the power received at port 2 ( $\left.\mathrm{P}_{\text {rec }}\right)$ in a two-port setup. In this case the implanted antennas have been considered as the transmitters, and the external antenna as the receiver. As external antenna the half-wave dipole has been selected to simplify the analysis and decrease the computational time of 


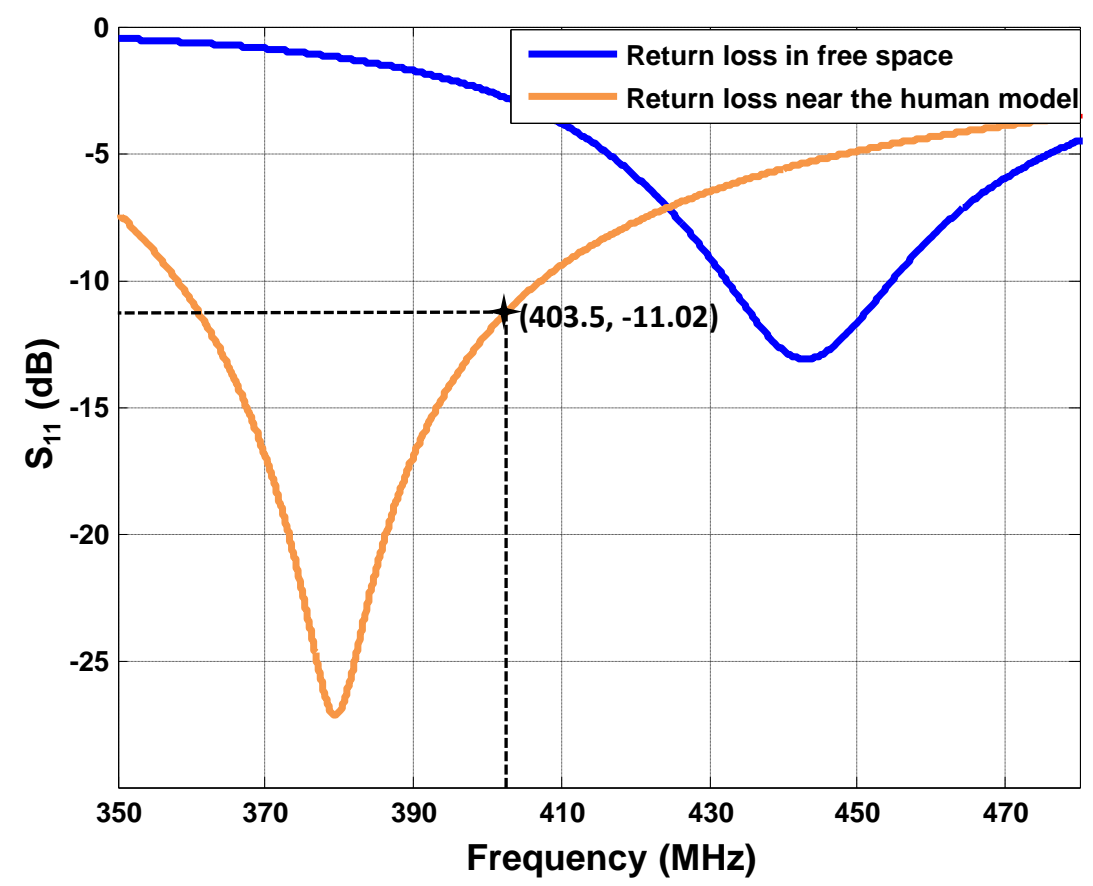

Figure 28. Return loss of the helical dipole antenna. Reused with permission from [92].

the simulations. The half-wave dipole has been placed in several positions around the arm, with a maximum distance from the skin of $10 \mathrm{~mm}$ (as in Figure 24), to simulate possible locations for the antenna in the socket prosthesis. This represents a typical scenario of transmission from in-body to body surface.

Considering as reference distance $d_{0}=25 \mathrm{~mm}$ and the following expression:

$$
P L_{d B}(d)=-\left|S_{21}\right|_{d B}
$$

the path loss has been modeled, finding the values for $P L_{0}, n$ and $\mathrm{S}$, as defined in equation 6.5. The mean value of the path loss has been obtained by fitting a least square regression line through the scatter of measured path loss points in $\mathrm{dB}$. The coefficients of the regression have been obtained with a $95 \%$ coefficient bounds. The resulting parameters of the fitted simulating model are: $\mathrm{PL}_{0}=61.12, n=2.71, \sigma_{s}=5.10$. In Figure 30 are represented the values of the path loss as a function of the distance in several positions of the external 


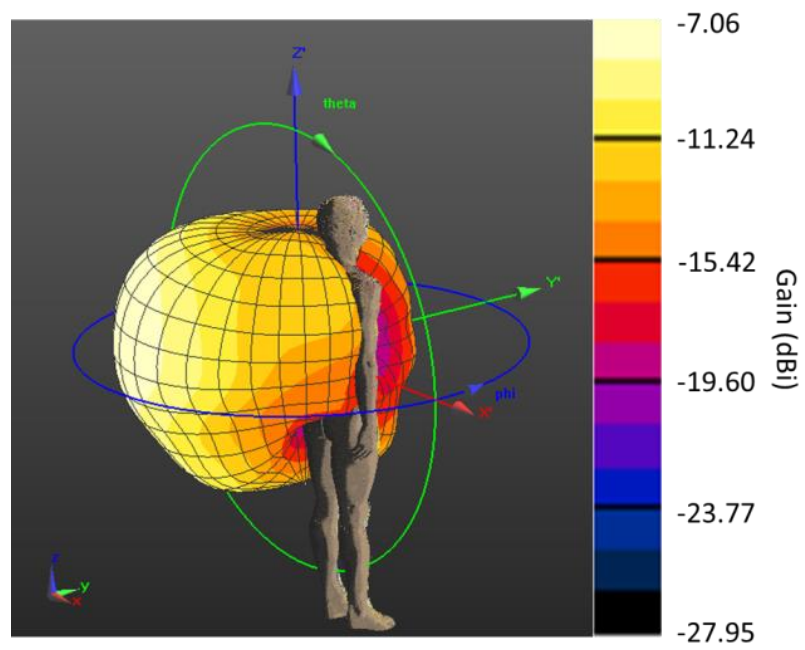

(a)

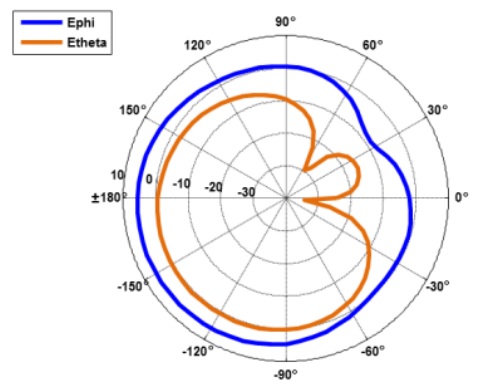

Plane XY

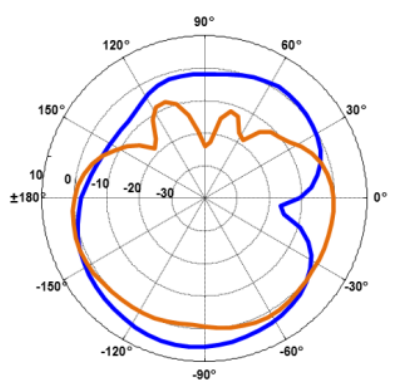

Plane XZ

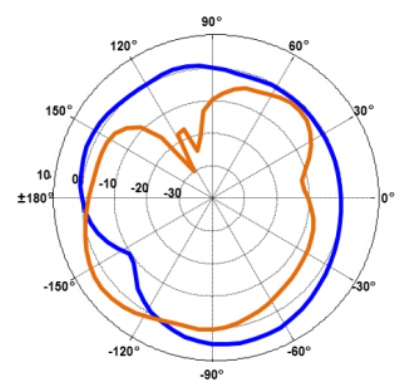

Plane YZ

(b)

Figure 29. 3D Gain polar and radiation pattern plots of the helical dipole antenna (a) 3D gain. (b) Values of $E_{\phi}(d B)$ and $E_{\theta}(d B)$ in three different planes. The coordinate system is shown in figure. Reused with permission from [92].

antenna. The orange curve is the fitting curve obtained through a least square linear regression, while the blue dots are the values of the path loss. The model takes into account also the shadowing effect (the term S) as considered in the equation 6.5. $\mathrm{S}$ is a random variable with a normal distribution, zero mean and standard deviation $\sigma_{s}$ and it occurs when the distance between the two antennas is the same, but they might have different positions or directions. The distances are in the range $25-80 \mathrm{~mm}$. Subsequently the half-wave dipole has been substituted with the helical antenna and few more simulations have been done. 


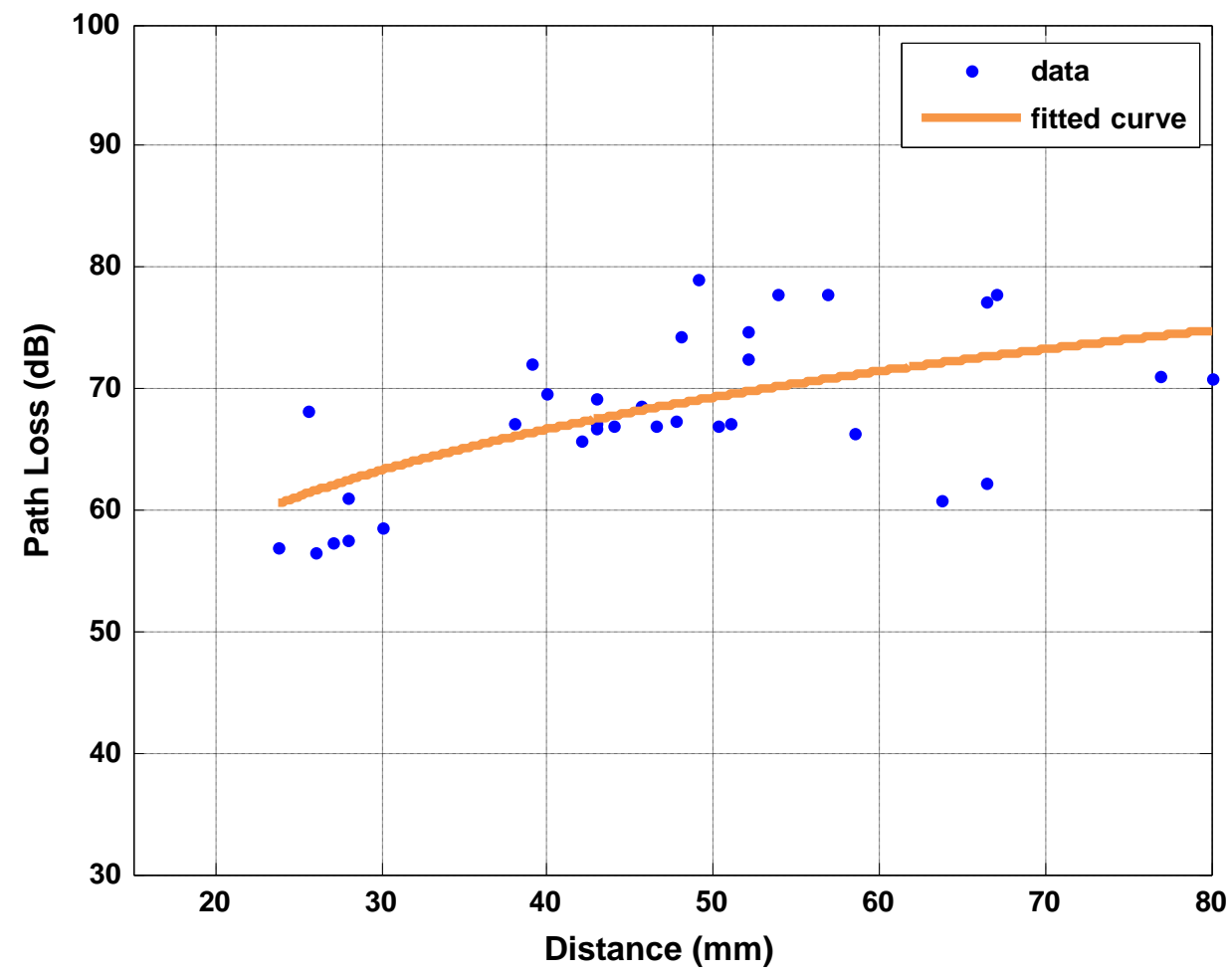

Figure 30. Path loss values as function of the distance between the implanted antenna and the external antenna and representation of the fitted model. Reused with permission from [92].

The values of the path loss obtained are in line with the model built considering the dipole. In Table 3 the values and the corresponding distances are listed.

Table 3: path loss values with dipole helical antenna

\begin{tabular}{|l|l|}
\hline Distance $(\mathbf{m m})$ & $\begin{array}{l}\text { Path Loss } \\
(\mathrm{dB})\end{array}$ \\
\hline 38 & 62.98 \\
\hline 43 & 61.68 \\
\hline 65 & 72.55 \\
\hline 69 & 74.89 \\
\hline
\end{tabular}




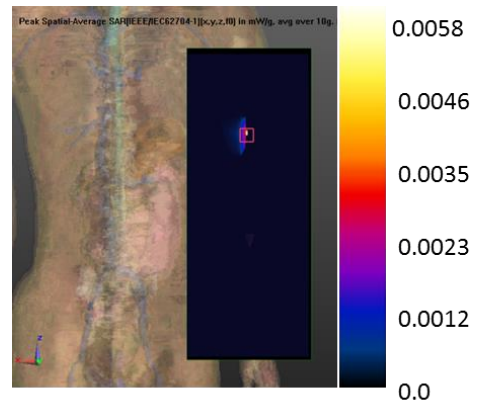

(a)

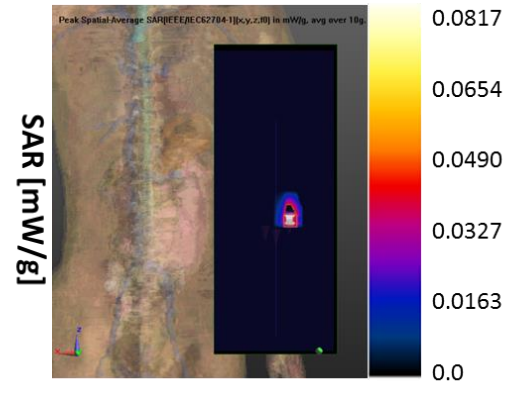

(b)

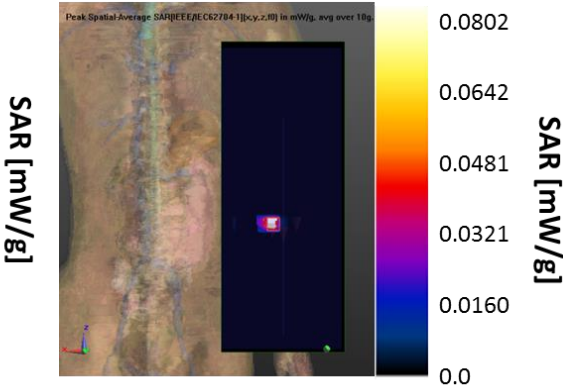

(c)

Figure 31. psaSAR values with the half wave dipole. a) psaSAR related to the external half-wave dipole positioned in front of the arm (scenario 1); b) psaSAR related to implant 1 (scenario 1); c) psaSAR related to implant 2 (scenario1). Reused with permission from [92].

\subsection{Specific Absorption Rate}

The induced SAR has been calculated with the software SEMCAD X and with the 3D human body model Duke. It has been evaluated the spatial peak average SAR based on the IEEE/IEC62704-1 standard [94], using a cube of mass of lossy tissue (normally $1 \mathrm{~g}$ or $10 \mathrm{~g}$ ) to average SAR value. The SAR has been evaluated for two different scenarios, considering the two implanted antennas and the half-wave dipole. In a third scenario the SAR has been calculated also with the helical antenna and one implanted antenna. The peak spatial average SAR (psaSAR) averaged over $10 \mathrm{~g}$ of tissues and normalized to $1 \mathrm{~mW}$ input power for the implanted antennas has been evaluated for all scenarios according with [94].

In Figure 31 are represented the SAR values for the first scenario. The half-wave dipole is positioned in front of the arm, at $\sim 10 \mathrm{~mm}$ from the skin. In Figures 31a, 31b and 31c are shown the SAR values for the external antenna and for the two implants respectively. The red square is the position of the peak value. The peak value for the external antenna has a value of $0.00577576 \mathrm{~mW} / \mathrm{g}$ and is located on the skin of the arms where the dipole has minimum distance. For both implants the peaks (the red square) are located on the tissues that are close to the antenna. The psaSAR values are $0.0817047 \mathrm{~mW} / \mathrm{g}$ (Figure $31 \mathrm{~b}$ ) and $0.0802022 \mathrm{~mW} / \mathrm{g}$ (Figure 31c). All the values are lower than the limitation imposed by ICNIRP (4 W/Kg). 


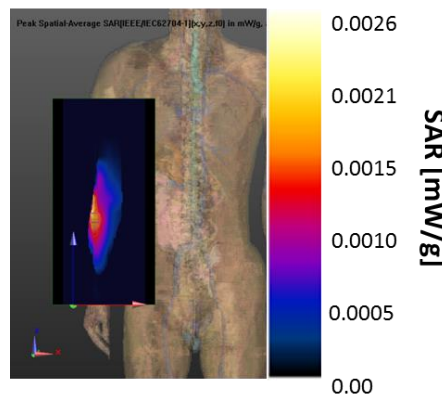

(a)

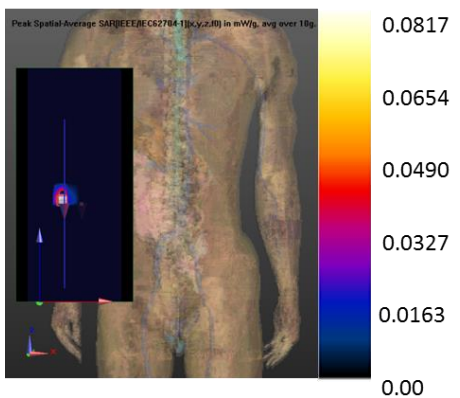

(b)

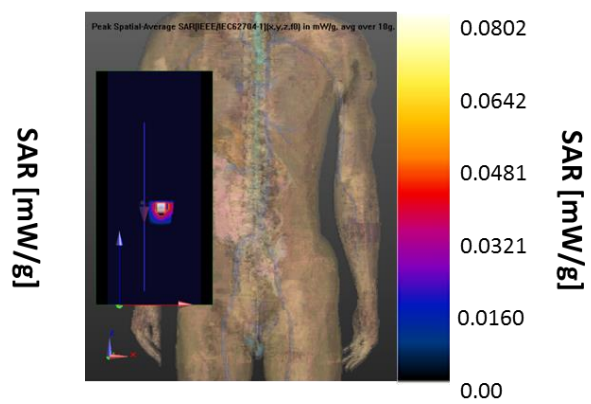

(c)

Figure 32. (a) psaSAR related to the external half-wave dipole positioned on the back of the arm (scenario2); (b) psaSAR related to implant 1(scenario2); (c) psaSAR related to implant 2 (scenario2). Reused with permission from [92].

A second scenario with the external dipole antenna positioned in the back of the arm has been evaluated. The distance between the external antenna and the skin is also in this case around $10 \mathrm{~mm}$. The external antenna psaSAR is located few millimeters under the skin in the back of the arm in correspondence of the dipole source and is $0.00257913 \mathrm{~mW} / \mathrm{g}$ (Figure 32a). The peak values of the implants are $0.0817043 \mathrm{~mW} / \mathrm{g}$ and $0.0802018 \mathrm{~mW} / \mathrm{g}$ (Figures $32 \mathrm{~b}$ and $32 \mathrm{c}$ ). It has to be noticed that the psaSAR values measured in the two scenarios are very close to each other, and they are quite far from the ICNRP limitations. Considering the psaSAR values obtained and the ICNIRP limitation for limbs $(4 \mathrm{~W} / \mathrm{kg})$, the maximum input power allowable results $\sim 50 \mathrm{~mW}$. A further analysis has been conducted with the helical dipole antenna. In this case, to shorten the computational time (that can take more than one week), only one implanted antenna has been considered. The psaSAR for the helical dipole antenna is $0.00350877 \mathrm{~mW} / \mathrm{g}$ and is located on the side of the arm, close to the source (Figure 33a), while for the implant is $0.0802028 \mathrm{~mW} / \mathrm{g}$ and it is located on the on the tissues near the implant (Figures 33b). This confirm that the input power could be increased up to $\sim 50 \mathrm{~mW}$. 


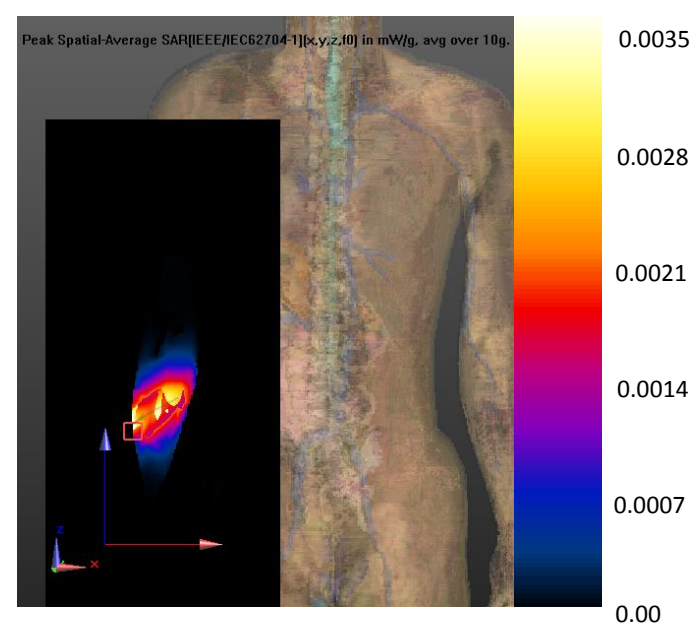

(a)

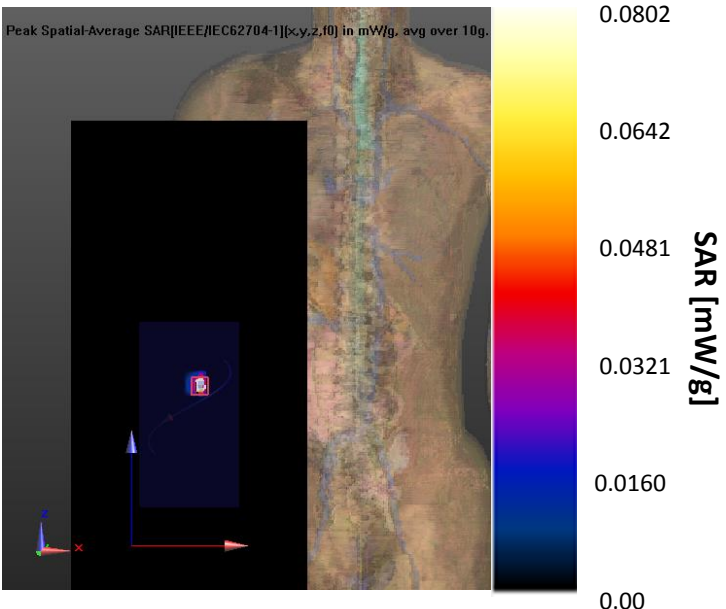

(b)

Figure 33. (a) psaSAR related to the external helical dipole; (b) psaSAR related to implant 1

\subsection{Link budget analysis}

The link budget analysis can validate the feasibility of a wireless link. The channel modeling and the characteristics of the antennas, both implanted and external, that have been described in the previous paragraphs can provide the necessary data for a first link budget analysis. Along with the data it is need take into account the limitations limitation provided by the ITU recommendation for MICS bandwidth [95] and by the standard for WBAN [74]. In Table 4 is presented the preliminary link budget analysis based on this study. It has been

considered a symbol rate of $151.5 \mathrm{kbps}$ as suggested by IEEE802.15.6 [74]. The path loss values have been obtained from the model obtained above which take into account the influence of the human body and the fading. The received power $P_{R}$ has been calculated as:

$$
P_{R}=E I R P-P L+G_{R} \quad(d B m)
$$

where EIRP $[\mathrm{dBm}]$ is the effective isotropic radiated power, including the input power and the transmitting implanted antenna gain. For MICS systems EIRP $=-16 \mathrm{dBm}$, according with [95]. $P L[d B]$ is the path loss, it has been considered for a distance of $45 \mathrm{~mm}\left(68 \mathrm{~dB}\right.$ ), and $\mathrm{G}_{R}$ $[\mathrm{dBi}]$ is the receiver antenna gain. As receiver antenna it has been considerd the half-wave 
dipole with $G_{R}=-3.20 \mathrm{dBi}$ (see Figure 22). The maximum transmit power from equation 4.2 is $P_{R}=-87.2 \mathrm{dBm}$.

The receiver sensitivity $S_{R}$ is defined as:

$$
S_{R}=S N R+N_{0}+B W+N F+L_{R}(d B m)
$$

where SNR $[d B]$ is the signal to noise ratio, $N_{0}[d B / H z]$ is the thermal noise density for the implant (considering the temperature of the body $37^{\circ} \mathrm{C}$ ), BW [dB] is the bandwidth (calculated as $\left.10^{*} \log (B W[H z])\right), N F[d B]$ is the noise figure, and $L_{R}[d B]$ are the losses at the receiver. The receiver sensitivity from equation 4.3 is $S_{R}=-98.2 \mathrm{dBm}$.

Finally the link margin (LM) is defined as

$$
L M=P_{R}-S_{R} \quad(d B) \quad(4.4)
$$

and it results $L M=11 \mathrm{~dB}$.

Table 4: link budget

\begin{tabular}{|l|l|}
\hline PARAMETERS (up-link) & Value \\
\hline Frequency & $402-405 \mathrm{MHz}$ \\
\hline Modulation type & $\pi / 2-\mathrm{DBPSK}$ \\
\hline Data rate & $151.8 \mathrm{kbps}$ \\
\hline BW Bandwidth & $300 \mathrm{kHz}$ \\
\hline SNR & $5 \mathrm{~dB}$ \\
\hline NF Noise Figure & $10 \mathrm{~dB}$ \\
\hline $\mathrm{L}_{R}$ losses at the receiver & $6 \mathrm{~dB}$ \\
\hline $\begin{array}{l}\mathrm{N}_{0} \text { Thermal noise density for } \\
\text { implant }\end{array}$ & $-174 \mathrm{dBm} / \mathrm{Hz}$ \\
\hline PL Path Loss (d=45 mm) \\
including fading
\end{tabular}




\begin{tabular}{|l|l|}
\hline (dipole) & \\
\hline EIRP & $-16 \mathrm{dBm}$ \\
\hline$P_{R}$ Received power & $-87.2 \mathrm{dBm}$ \\
\hline$S_{R}$ Receiver sensitivity & $-98.2 \mathrm{dBm}$ \\
\hline LM Link Margin & $11 \mathrm{~dB}$ \\
\hline
\end{tabular}

The link budget analysis is summarized in Table 4. The system consisting of two devices in a forearm of a human and one on-body device placed near the skin of the arm has a link margin of $11 \mathrm{~dB}$. This value of the link margin is very good considering that the gain of the implanted antenna and the path loss can vary across the subjects. The gain of the external antenna can also be improved selecting a more efficient antenna. In this study the low value of the implanted antenna gain limits the received power. This limitation can be overtake setting a higher transmit power, which is not desirable in terms of safety (SAR limits) and battery consumption. To improve the quality of link the gain of both type of antennas can be improved along with a modulation scheme.

\subsection{Conclusions}

The wireless link that has been characterized in this study represents the first step to build a WBAN that can be used in the field of upper limb prostheses. The WBAN will consist of 2 or more, depending on the needs, implanted devices capable to record, process and transmit EMG signals to an on-body device that will be placed inside the socket of the prosthesis. The on-body device will receive the processed signal from the implants and will exploit them to control the prosthesis. Moreover if necessary the signals can be sent to a computer that can be placed not more than $2 \mathrm{~m}$ distance. The wireless RF link has been characterized and modeled with the limitation imposed by IEEE802.15.6 [74] and by ITU [95]. The safety in terms of SAR has been investigated, finding values much lower than the limitations imposed by ICNRP. Finally a preliminary link budget analysis has been performed providing a link margin of $11 \mathrm{~dB}$, which results to be a good margin for the implementation. This link margin 
could be further increased designing antenna with a higher gain and using a different modulation scheme. A different modulation scheme can be chosen to also increase the data rate.

The main conclusion of this study concerns the use of the standard communication protocol (IEEE802.15.6) and the use of a dedicated band (MICS). These two aspects are very interesting for industries that are developing upper limb prosthesis. Indeed, they can develop wireless systems with a standard communication protocol without take care of the interferences.

The system proposed has the advantage respect to other wireless system in literature that can be used with each type of amputee, there no constraints for the positioning of the implants. The number of wireless implants can be increased depending on the needs. Moreover this technology is not limited to EMG sensors, the same results presented here are valid also in the case of nerve electrodes, which can be used for sensory feedback.

Novel algorithms that can use the information coming from implants are also needed. Further investigations are needed to improve the system, nevertheless this study is the necessary first step to confirm that such system can be actually implemented and further developed to provide a new generation of upper limb prostheses in the future, which will be more reliable and robust. 


\section{Summary and conclusions}

The main progresses in the prosthetic field were made following World War II. Most upper limb prostheses currently on the market are based on the concepts developed at that time. On the other side, in the last few years the technology has advanced substantially and has provided prostheses that replace several DoFs. Yet, the patients are hesitant to use them. The reasons depend on the fact that these new generations of upper limb prostheses do not have an intuitive control and often they lack in robustness and reliability. Starting from these considerations, this thesis has focused on how to increase robustness and reliability. The work was in two parts. The first part focused on a non-invasive approach, based on surface EMG electrodes, as in all the commercially available prostheses. The second part focused on an invasive approach. A new system has been described and designed based on implanted EMG sensors.

The first part of the thesis starts with the state of the art of upper limb prostheses with sEMG and of the pattern recognition algorithms that have been proposed in literature. The main issues have been identified, as the shift of the electrodes when donning and doffing the prosthesis and the presence of noisy channels. The study that has been conducted to advance the state of the art, maintaining a non-invasive approach, is based on the use of spatial correlation of surface HD EMG. A measure of spatial correlation has been used as feature in the pattern recognition algorithm. To prove that the new method proposed can increase robustness and reliability a set of data has been collected from 7 able-bodied subjects and on one amputee subject. A matrix of 192 EMG channels has been placed on the forearm of the able-bodied subjects, while for the amputee the matrix was with 144 channels, due to his short stump. The subjects performed nine different tasks. The tasks are: wrist flexion, wrist extension, radial deviation, ulnar deviation, forearm pronation, forearm supination, hand open, hand closing, and the rest position (9 classes). The amputee performed 7 tasks, the same as able-bodied but hand open and hand closing. The study concluded that the new feature, based on spatial correlation, reduces the sensitivity to electrodes shifts respect to classical features used in literature. Moreover, the method 
proposed allowed to eliminate channels during the test phase without re-training, maintaining a good classification accuracy for a relatively large proportion of omitted channels.

This method has the potentiality to become a standard in the future, but technological limitations needs to be addressed. The method considers the exploitation of hundreds of electrodes that have to be amplified and processed with instrumentations that have to be enclosed in the robotic hand, that means that have to be relatively small. Another important aspect to be considered concerns the HD EMG electrodes. They need to support long term usage, that means good skin contact and no degradation of the signals. In chapter 4 the most recent studies which are exploiting HD EMG and spatial correlation have been considered, highlighting the increasing interest on this technology and in the advancements in microprocessors dedicated to control of upper limb prostheses with HD EMG.

In the second part of the thesis it has been considered an invasive approach for improving the control of upper limb prosthesis. This means that the EMG signals to control the prosthesis are acquired with devices that are implanted directly in the muscle. This solution allows to overcome the shift of the electrodes, since a device implanted is anchored to the muscle, furthermore it is possible acquire information directly into the muscle that has to give the control command.

The state of the art of implanted EMG and of implanted devices and technology available in general has been analyzed. In has been stated that in literature there are few proposals and none of them has been commercialized yet. The main points to consider for a wireless RF solution have been carried out. Taking into account this a new solution has been proposed. It includes the wireless transmission of EMG signals recorded inside the human body. The solution proposed is based on a standard communication protocol (IEEE 802.15.6) and consists in two implanted devices that transmit and process the EMG signal acquired to a controller placed in the socket of the prosthesis. In this thesis has been defined the channel model, considering the two implants and an external antenna, by electromagnetic simulations. The safety of the proposed system has been verified, measuring the SAR. 
Finally a preliminary link budget analysis has been done, confirming the feasibility of the system.

The presented results are the first steps for the realizations of the proposed system. Many other steps are necessary for the final implementation, including new control algorithms that can better exploit the information acquired by the implants. Nevertheless, here it has been demonstrated that the use of implanted EMG can represent a valid solution in the field of upper limb prostheses. This type of solutions presents limitations that have to be carefully considered. The implants are chronic, so they need to be encapsulated into the human tissues, further the maintenance (for example change of batteries) as to be minimal, done to a distance of years. The research in the field of implanted device is very active and soon the technology will be ready also for applications in robotic prostheses.

This thesis addressed two approaches with the intent of improving the robustness in upper limb prostheses. The scope has not been to find the best approach, but to provide two different points of view of the same problem. The choice between the two approaches depends on several factors that are mainly related to the practical translation of the methods into systems that can be reliably used for daily routine activities. 


\section{References}

[1] WorkSafeBC Evidence-Based Practice Group and Dr. W. M. Craig, "Upper Limb Prostheses A Review of the Literature With a Focus on Myoelectric Hands," Clin. Serv. Work. Empl. Serv., no. February, 2011.

[2] A. Freeland, R. Psonak "Traumatic Below-elbow Amputations". ORTHOPEDICS. 2007; 30: doi: 10.3928/01477447-20070201-16

[3] E. J. MacKenzie, "Limb amputation and limb deficiency: epidemiology and recent trends in the United States." The Free Library 01 August 2002. 28 June 2016.

[4] J. Settakorn, S. Rangdaeng, O. Arpornchayanon, S. Lekawanvijit, L. Bhoopat, and J. Attia, "Why were limbs amputated? An evaluation of 216 surgical specimens from Chiang Mai University Hospital, Thailand," Arch. Orthop. Trauma Surg., vol. 125, no. 10, pp. 701705, Dec. 2005.

[5] F. Cordella, A. L. Ciancio, R. Sacchetti, A. Davalli, A. G. Cutti, E. Guglielmelli, and L. Zollo, "Literature Review on Needs of Upper Limb Prosthesis Users," Front. Neurosci., vol. 10, no. May, pp. 1-14, 2016.

[6] K. Norton, "A brief History of Prosthetics," InMotion, vol. 17, no. 7, pp. 1-3, 2007.

[7] V. Putti, "Scritti Medici", La chirurgia degli organi di movimento IX.4-5, 1925. (Reprint) “Historical prostheses," J. Hand Surg. Am., vol. 30, no. 3, pp. 310-325, 2005.

[8] A. E Kobrinski, et al. "Problems of bioelectric control." Automatic and Remote Control, Proc. 1stIFAC Int. Cong 2 (1960): 619.

[9] E. A. Biddiss and T. T. Chau, "Upper limb prosthesis use and abandonment: a survey of the last 25 years.," Prosthet. Orthot. Int., vol. 31, no. 3, pp. 236-257, 2007.

[10] C. Pylatiuk, S. Schulz, and L. Döderlein, "Results of an Internet survey of myoelectric prosthetic hand users.," Prosthet. Orthot. Int., vol. 31, no. 4, pp. 362-70, 2007.

[11] B. Graimann, H Dietl, "Introduction to Upper Limb Prosthetics", In: Introduction to Neural Engineering for Motor Rehabilitation. John Wiley \& Sons, Inc., Chap 14, pp 267-290.

M. Atzori and H. Müller, "Control Capabilities of Myoelectric Robotic Prostheses by Hand Amputees: A Scientific Research and Market Overview.," Front. Syst. Neurosci., vol. 9, no. November, p. 162, 2015. 
[13] D. Farina, Ning Jiang, H. Rehbaum, A. Holobar, B. Graimann, H. Dietl, and O. C. Aszmann, "The Extraction of Neural Information from the Surface EMG for the Control of Upper-Limb Prostheses: Emerging Avenues and Challenges," IEEE Trans. Neural Syst. Rehabil. Eng., vol. 22, no. 4, pp. 797-809, Jul. 2014.

[14] N. Jiang, S. Dosen, K.-R. Müller, and D. Farina, "Myoelectric Control of Artificial Limbs- Is There a Need to Change Focus?," IEEE SIGNAL PROCESSING MAGAZINEEEE SIGNAL PROCESSING MAGAZINE, no. SEPTEMBER, pp. 148-152, 2012.

[15] B. Hudgins, P. Parker, and R. N. Scott, "A new strategy for multifunction myoelectri control," IEEE Trans. Biomed. Eng., vol. 40, no. 1, pp. 5541-548, 1993.

E. Scheme and K. Englehart, "Electromyogram pattern recognition for control of powered upper-limb prostheses: State of the art and challenges for clinical use," J. Rehabil. Res. Dev., vol. 48, no. 6, pp. 643-660, 2011.

[17] L. H. Smith, L. J. Hargrove, B. A. Lock, and T. A. Kuiken, "Determining the optimal window length for pattern recognition- based myoelectric control: balancing the competing effects of classification error and controller delay," IEEE Trans Neural Syst Rehabil Eng, vol. 19, no. 2, pp. 186-192, 2011.

[18] D. Tkach, H. Huang, and T. A. Kuiken, "Study of stability of time-domain features for electromyographic pattern recognition.," J. Neuroeng. Rehabil., vol. 7, no. 1, p. 21, 2010.

[19] T. W. Williams, III, "Practical methods for controlling powered upper extremity prostheses," Assistive Technol., vol. 2, pp. 3-18, 1990

[20] T. R. Farrell and R. F. Weir, "A Comparison of the Effects of Electrode Implantation and Targeting on Pattern Classification Accuracy for Prosthesis Control," IEEE Trans. Biomed. Eng, vol. 55, no. 9, pp. 2198-2211, 2008.

[21] K. Englehart and B. Hudgins, "A Robust, Real Time Control Scheme for Multifunction Myoelectric Control," IEEE Trans. Biomed. Eng., vol. 7, no. 7, pp. 848-854, 2003.

[22] M. Zecca, S. Micera, M. C. Carrozza, and P. Dario, "Control of multifunctional prosthetic hands by processing the electromyographic signal.," Crit. Rev. Biomed. Eng., vol. 30, no. 4-6, pp. 459-85, 2002. 
[23] M. Zardoshti-Kermani, B. C. Wheeler, K. Badie, and R. M. Hashemi, "EMG feature evaluation for movement control of upper extremity prostheses," IEEE Trans. Rehabil. Eng., vol. 3, no. 4, pp. 324-333, 1995.

[24] K. Englehart, B. Hudgins, P. Parker, and M. Stevenson, "Time-frequency representation for classification of the transient myoelectric signal," Proc. 20th Annu. Int. Conf. IEEE Eng. Med. Biol. Soc. Vol.20 Biomed. Eng. Towar. Year 2000 Beyond (Cat. No.98CH36286), vol. 5, no. 5, pp. 2627-2630, 1998.

[25] P. Zhou, M. M. Lowery, K. B. Englehart, H. Huang, G. Li, L. Hargrove, J. P. A. Dewald, and T. A. Kuiken, "Decoding a new neural machine interface for control of artificial limbs," J. Neurophysiol., vol. 98, no. 5, pp. 2974-82, 2007.

[26] L. Hargrove, S. Member, K. Englehart, S. Member, and B. Hudgins, "A Comparison of Surface and Intramuscular Myoelectric Signal Classification," vol. 54, no. 5, pp. 847-853, 2006.

[27] A. J. Young, L. J. Hargrove, and T. A. Kuiken, "The effects of electrode size and orientation on the sensitivity of myoelectric pattern recognition systems to electrode shift," IEEE Trans. Biomed. Eng., vol. 58, no. 10, pp. 2537-2544, Oct. 2011.

[28] L. Hargrove, K. Englehart, and B. Hudgins, "A training strategy to reduce classification degradation due to electrode displacements in pattern recognition based myoelectric control," Biomed. Signal Process. Control, vol. 3, no. 2, pp. 175-180, 2008.

[29] A. Young, L. J. Hargrove, and T. A. Kuiken, "The effects of electrode size and orientation on the sensitivity of myoelectric pattern recognition systems to electrode shift," ... , IEEE Trans., vol. 58, no. 9, pp. 2537-2544, 2011.

[30] A. Stango, F. Negro, and D. Farina, "Spatial Correlation of High Density EMG Signals Provides Features Robust to Electrode Number and Shift in Pattern Recognition for Myocontrol," IEEE Trans. Neural Syst. Rehabil. Eng., vol. 23, no. 2, pp. 189-198, 2015.

[31] A. J. Young, L. J. Hargrove, and T. a Kuiken, "Improving myoelectric pattern recognition robustness to electrode shift by changing interelectrode distance and electrode configuration.," IEEE Trans. Biomed. Eng., vol. 59, no. 3, pp. 645-52, Mar. 2012. 
[32] R. Merletti, A. Holobar, and D. Farina, "Analysis of motor units with high-density surface electromyography.," J. Electromyogr. Kinesiol., vol. 18, no. 6, pp. 879-90, Dec. 2008.

[33] R. Merletti, P.A. Parker, editors. Electromyography: Physiology, Engineering and Noninvasive Applications. 1st ed. Hoboken, NJ: John Wiley \& Sons, Inc.; 2004

[34] H. Daley, K. Englehart, L. Hargrove, and U. Kuruganti, "High density electromyography data of normally limbed and transradial amputee subjects for multifunction prosthetic control.," J. Electromyogr. Kinesiol., vol. 22, no. 3, pp. 478-84, Jun. 2012.

[35] S. Muceli and D. Farina, "Simultaneous and proportional estimation of hand kinematics from EMG during mirrored movements at multiple degrees-of-freedom.," IEEE Trans. Neural Syst. Rehabil. Eng., vol. 20, no. 3, pp. 371-8, May 2012.

[36] S. Muceli, N. Jiang, and D. Farina, "Extracting signals robust to electrode number and shift for online simultaneous and proportional myoelectric control by factorization algorithms," IEEE Trans. Neural Syst. Rehabil. Eng., vol. 22, no. 3, pp. 623-633, May 2014.

[37] P. Zhou, M. Lowery, J. A Dewald, and T. Kuiken, "Towards Improved Myoelectric Prosthesis Control: High Density Surface EMG Recording After Targeted Muscle Reinnervation.," Conf. Proc. IEEE Eng. Med. Biol. Soc., vol. 4, pp. 4064-7, 2005.

[38] H. Huang, P. Zhou, G. Li, and T. Kuiken, "Spatial filtering improves EMG classification accuracy following targeted muscle reinnervation," Ann. Biomed. Eng., vol. 37, no. 9, pp. 1849-1857, 2009.

[39] H. Huang, P. Zhou, G. Li, and T. A. Kuiken, "An analysis of EMG electrode configuration for targeted muscle reinnervation based neural machine interface," IEEE Trans. Neural Syst. Rehabil. Eng., vol. 16, no. 1, pp. 37-45, 2008.

[40] D. Farina, H. Rehbaum, A. Holobar, I. Vujaklija, N. Jiang, C. Hofer, S. Salminger, H.-W. van Vliet, and O. C. Aszmann, "Noninvasive, accurate assessment of the behavior of representative populations of motor units in targeted reinnervated muscles.," IEEE Trans. Neural Syst. Rehabil. Eng., vol. 22, no. 4, pp. 810-9, Jul. 2014. 
[41] M. Umer, L. Kulik, and E. Tanin, "Spatial interpolation in wireless sensor networks: Localized algorithms for variogram modeling and Kriging," Geoinformatica, vol. 14, no. 1, pp. 101-134, 2010.

[42] B. Akan, I. F. Akyildiz, and M. C. Vuran, "Spatio-temporal correlation : theory and applications for wireless sensor networks," vol. 45, pp. 245-259, 2004.

[43] S. Pattem, B. Krishnamachari, and R. Govindan, "The impact of spatial correlation on routing with compression in wireless sensor networks," ACM Trans. Sens. Networks, vol. 4, no. 4, p. 24, 2008.

[44] H. Hu and Z. Yang, "Spatial Correlation-Based Distributed Compressed Sensing in Wireless Sensor Networks," International Conference on Wireless Communications, Networking and Mobile Computing - WiCom, 2010. DOI: 10.1109/WICOM.2010.5601158.

[45] M. C. Vuran and I. F. Akyildiz, "Spatial correlation-based collaborative medium access control in wireless sensor networks," IEEE/ACM Trans. Netw., vol. 14, no. 2, pp. 316329, Apr. 2006.

[46] R. Webster and M. A. Oliver, Geostatistics for Environmental Scientists. John Wiley \& Sons, 2007.

[47] R. A. Olea, "A six-step practical approach to semivariogram modeling," Stoch. Environ. Res. Risk Assess., vol. 20, no. 5, pp. 307-318, May 2006.

[48] C. Chang and C. Lin, "LIBSVM: a library for support vector machines," ACM Transaction on Intelligent Systems and Technology (TIST), vol. 2, pp. 27, 2011.

[49] L. Hargrove, Y. Losier, B. Lock, K. Englehart, and B. Hudgins, "A real-time pattern recognition based myoelectric control usability study implemented in a virtual environment.," Conf. Proc. IEEE Eng. Med. Biol. Soc., vol. 2007, pp. 4842-5, Jan. 2007.

[50] H. R. Marateb, M. Rojas-Martínez, M. Mansourian, R. Merletti, and M. A. M. Villanueva, "Outlier detection in high-density surface electromyographic signals," Med. Biol. Eng. Comput., vol. 50, no. 1, pp. 79-89, 2012.

[51] I. Vujaklija, D. Farina, and O. C. Aszmann, "New developments in prosthetic arm systems," Orthop. Res. Rev., vol. 8, p. 31-39, 2016. 
[52] L. Pan, D. Zhang, N. Jiang, X. Sheng, and X. Zhu, "Improving robustness against electrode shift of high density EMG for myoelectric control through common spatial patterns," J. Neuroeng. Rehabil., vol. 12, no. 1, p. 110, 2015.

[53] J.M. Hahne, B. Graimann, K. Muller, "Spatial filtering for robust myoelectric control", IEEE Trans Biomed Eng. 2012;59(5):1436-1443.

[54] G. Huang, Z. Zhang, D. Zhang, X. Zhu, "Spatio-spectral filters for low-density surface electromyographic signal classification," Medical \& biological engineering \& computing. 2013;51(5):547-55.

[55] N. Celadon, S. Došen, I. Binder, P. Ariano, and D. Farina, "Proportional estimation of finger movements from high-density surface electromyography," J. Neuroeng. Rehabil., vol. 13, no. 1, p. 73, 2016.

[56] X. Hu, N. L. Suresh, C. Xue, and W. Z. Rymer, "Extracting extensor digitorum communis activation patterns using electromyography," vol. 6, no. October, pp. 1-9, 2015.

[57] M. Jordanic, M. Rojas-Martínez, M. A. Mañanas, and J. F. Alonso, "Spatial distribution of HD-EMG improves identification of task and force in patients with incomplete spinal cord injury," J. Neuroeng. Rehabil., vol. 13, no. 1, pp. 1-11, 2016.

[58] A. Boschmann, A. Agne, L. Witschen, G. Thombansen, F. Kraus, and M. Platzner, "FPGA-based acceleration of high density myoelectric signal processing," in 2015 International Conference on ReConFigurable Computing and FPGAs (ReConFig), 2015, pp. 18.

[59] A. Boschmann, A. Agne, L. Witschen, G. Thombansen, F. Kraus, and M. Platzner, "Zynq-based Acceleration of Robust High Density Myoelectric Signal Processing," submitted to The Journal of Parallel and Distributed Computing, July 2016

[60] A. Boschmann, A. Stango, G. Thombansen, A. Wiens, D. Farina, M. Platzner, "Robust Computer Vision-based Features for HD EMG Prosthesis Control", (in preparation).

[61] D. G. Lowe, “Object recognition from local scale-invariant features," Proc. Seventh IEEE Int. Conf. Comput. Vis., vol. 2, no. [8, pp. 1150-1157, 1999.

[62] S. Lazebnik, C. Schmid, and J. Ponce, "Beyond Bags of Features: Spatial Pyramid Matching for Recognizing Natural Scene Categories," in 2006 IEEE Computer Society 
Conference on Computer Vision and Pattern Recognition - Volume 2 (CVPR'06), 2006, vol. 2, pp. 2169-2178.

[63] A. Bosch, A. Zisserman, and X. Munoz, "Image Classification using Random Forests and Ferns," in 2007 IEEE 11th International Conference on Computer Vision, 2007, pp. 1-8.

[64] M. Ortiz-Catalan, R. Brånemark, B. Håkansson, and J. Delbeke, "On the viability of implantable electrodes for the natural control of artificial limbs: Review and discussion," Biomed. Eng. Online, vol. 11, no. 1, p. 33, 2012.

[65] K. D. Bergmeister, M. Hader, S. Lewis, M.-F. Russold, M. Schiestl, K. Manzano-Szalai, A. D. Roche, S. Salminger, H. Dietl, and O. C. Aszmann, "Prosthesis Control with an Implantable Multichannel Wireless Electromyography System for High-Level Amputees," Plast. Reconstr. Surg., vol. 137, no. 1, pp. 153-162, 2016.

[66] S. Lewis, M. Russold, H. Dietl, R. Ruff, J. M. C. Audí, K. P. Hoffmann, L. Abu-Saleh, D. Schroeder, W. H. Krautschneider, S. Westendorff, A. Gail, T. Meiners, and E. Kaniusas, "Fully implantable multi-channel measurement system for acquisition of muscle activity," IEEE Trans. Instrum. Meas., vol. 62, no. 7, pp. 1972-1981, 2013.

[67] B. D. Farnsworth, D. M. Talyor, R. J. Triolo, and D. J. Young, "Wireless in vivo EMG sensor for intelligent prosthetic control," in TRANSDUCERS 2009 - 15th International Conference on Solid-State Sensors, Actuators and Microsystems, 2009, pp. 358-361.

[68] IEEE Std 802.15.4a ${ }^{\mathrm{TM}}$-2007, IEEE Standard for Information TechnologyTelecommunications and Information Exchange Between Systems-LANs and MANsSpecific Requirements-Part 15.4: Wireless MAC and PHY Specifications for LR-WPANsAmendment 1: Add Alternate PHYs. IEEE Std 802.15.4a-2007.

[69] R. F. Weir, P. R. Troyk, G. A. DeMichele, D. A. Kerns, J. F. Schorsch, and H. Maas, "Implantable Myoelectric Sensors (IMESs) for Intramuscular Electromyogram Recording," IEEE Trans. Biomed. Eng., vol. 56, no. 1, pp. 159-171, Jan. 2009.

[70] P. F. Pasquina, M. Evangelista, A.J. Carvalho, J. Lockhart, S. Griffin, G. Nanos, P. McKay, M. Hansen, D. Ipsen, J. Vandersea, J. Butkus , M. Miller, I. Murphy, D. Hankin ," Firstin-man demonstration of a fully implanted myoelectric sensors system to control an advanced electromechanical prosthetic hand", Journal of Neuroscience Methods, 2014. 
[71] D. Farina and O. Aszmann, "Bionic Limbs: Clinical Reality and Academic Promises.," Sci. Transl. Med., vol. 6, no. 257, p. 257ps12, 2014.

[72] S. Movassaghi, M. Abolhasan, \& J. Lipman, "Wireless Body Area Networks: A Survey", 16(3), 1658-1686, 2014.

[73] K. Bazaka, M. \& Jacob, "Implantable Devices: Issues and Challenges. Electronics", Vol. 2, pp. 1-34, 2012. doi:10.3390/electronics2010001

[74] IEEE Standard for Local and metropolitan area networks Part 15.6: Wireless Body Area Networks, 10.1109/IEEESTD.2012.6161600, 2012

[75] P. Hall and Y. Hao, "Antennas and Propagation for Body-Centric Wireless Communications", IEEE Antennas and Propagation Magazine, Vol. 50, pp. 148-148, 2008. doi:10.1109/MAP.2008.4562277

[76] K. Yazdandoost \& R. Kohno, "An antenna for medical implant communications system", presented at Microwave Conference, 2007, (October), 968-971.

[77] Electromagnetic compatibility and Radio spectrum Matters (ERM); Short Range Devices (SRD); Ultra Low Power Active Medical Implants (ULP-AMI) and Peripherals (ULPAMI-P) operating in the frequency range $402 \mathrm{MHz}$ to $405 \mathrm{MHz}$; Part1: Techincal characteristics and test methods, ETSI EN 301 839-1 - V1.3.1, Vol. 1, pp. 1-52, 2009

[78] http://www.fcc.gov/encyclopedia/medical-device-radiocommunications-servicemedradio. Accessed July 2016

[79] ICNIRP Guidelines for Limiting Exposure To Time-Varying Electric, Magnetic and Electromagnetic Fields (up to 300GHz). Health Phys. 1998;74(4):494-522.

[80] ITU-T K.52: Guidance on complying with limits for human exposure to electromagnetic fields. (8/14)

[81] Health World Organization. Establishing a dialogue on risks from electromagnetic fields. Geneve; 2002.

[82] http://www.fcc.gov/guides/wireless-devices-and-health-concerns. Accessed 2 October 2014 
[83] IEEE P802.15-08-0780-10-0006, "Channel Model for Body Area Network (BAN)", https://mentor.ieee.org/802.15/dcn/08/15-08-0780-12-0006-tg6-channel-model.pdf,

Access 8 October 2014

[84] K. Sayrafian-Pour, W.-B. Yang, J. Hagedorn, J. Terrill, and K. Y. Yazdandoost, “A statistical path loss model for medical implant communication channels," in 2009 IEEE 20th International Symposium on Personal, Indoor and Mobile Radio Communications, 2009, pp. 2995-2999.

[85] E. Reusens, W. Joseph, B. Latré, B. Braem, G. Vermeeren, E. Tanghe, L. Martens, I. Moerman, and C. Blondia, "Characterization of on-body communication channel and energy efficient topology design for wireless body area networks.," IEEE Trans. Inf. Technol. Biomed., vol. 13, no. 6, pp. 933-45, Nov. 2009.

[86] A. Alomainy and Y. Hao, "Modeling and Characterization of Biotelemetric Radio Channel From Ingested Implants Considering Organ Contents," IEEE Trans. Antennas Propag., vol. 57, no. 4, pp. 999-1005, 2009.

[87] K. Yee, "Numerical solution of initial boundary value problems involving maxwell's equations in isotropic media," IEEE Trans. Antennas Propag., vol. 14, no. 3, pp. 302-307, May 1966.

[88] A. Christ, W. Kainz, E. G. Hahn, K. Honegger, M. Zefferer, E. Neufeld, W. Rascher, R. Janka, W. Bautz, J. Chen, B. Kiefer, P. Schmitt, H.-P. Hollenbach, J. Shen, M. Oberle, D. Szczerba, A. Kam, J. W. Guag, and N. Kuster, "The Virtual Family--development of surfacebased anatomical models of two adults and two children for dosimetric simulations.," Phys. Med. Biol., vol. 55, no. 2, pp. N23-N38, 2010.

[89] C. Gabriel: "Compilation of the dielectric properties of body tissues at RF and microwave frequencies", Report N.AL/OE-TR- 1996-0037, Occupational and environmental health directorate, Radiofrequency Radiation Division, Brooks Air Force Base, Texas (USA), June 1996

[90] Shmid \& Partner Engineering AG (SPEAG). (2000) SEMCAD. [Online].Available: http://www.SEMCAD.com 
[91] A. Stango, K. Y. Yazdandoost, and D. Farina, "Wireless Radio Channel for Intramuscular Electrode Implants in the Control of Upper Limb Prostheses," in EMBC 2015, 2015, pp. 4085-4088.

[92] A. Stango, K. Y. Yazdandoost, F. Negro, and D. Farina, "Characterization of In-Body to On-Body Wireless Radio Frequency Link for Upper Limb Prostheses", accepted PlosOne (2016)

[93] Kraus JD. Antennas. New York: McGraw Hill; 1988

[94] IEC/IEEE P62704-1/D2, IEC/IEEE Draft Standard for Determining the Peak Spatial Average Specific Absorption Rate (SAR) in the Human Body from Wireless Communications Devices, 30 MHz - 6 GHz. Part 1: General Requirements for using the Finite Difference Time. 2013. p. 1-64.

[95] ITU-R RS.1346. Sharing between the meteorological aids service and medical implant communication systems (MICS) operating in the mobile service in the frequency band 401-406 $\mathrm{MHz}$ 1998. p. 1-7. Available from: www.catr.cn/radar/itur/201007/P020100714434026745801.doc 


\section{List of dissemination during the PhD}

\section{Publications}

1. A. Stango, Y. K. Yazdandoost, F. Negro, D. Farina, "Characterization of In-Body to OnBody Wireless Radio Frequency Link for Upper Limb Prostheses", Plos One, under review

2. A. Stango, K. Y. Yazdandoost, and D. Farina, "Wireless Radio Channel for Intramuscular Electrode Implants in the Control of Upper Limb Prostheses," in EMBC 2015, 2015, pp. 4085-4088.

3. A. Stango, F. Negro, and D. Farina, "Spatial Correlation of High Density EMG Signals Provides Features Robust to Electrode Number and Shift in Pattern Recognition for Myocontrol," IEEE Trans. Neural Syst. Rehabil. Eng., vol. 23, no. 2, pp. 189-198, 2015

\section{Presentations in Conferences and Workshops}

1. Presentation in the $2^{\text {nd }}$ DEMOVE Symposium: High Density EMG technology for control of active prosthesis 
2. Conference IEEE Body Sensors networks, workshop "Implantable wireless sensors -from technology to deployment": SAR measurements in implanted devices controlling upper limb prostheses

\section{Publications in preparation}

C. Lin, A. Stango, N. Jiang, F. Negro, and D. Farina, "Robust Pattern Recognition Scheme to Electrode Number and Shift in Myocontrol with High Density EMG Signals"

A. Boschmann, A. Stango, G. Thombansen, A. Wiens, D. Farina, M. Platzner, "Robust Computer Vision-based Features for HD EMG Prosthesis Control" 


$\begin{aligned} \text { Address } & \text { am Kalten Born 59 20G } \\ & 37085 \text { Goettingen Geismar } \\ \text { Mobile } & +393288190386 \\ & +4915773962010 \\ \text { E-mail } & \text { antonietta.stango@gmail.com } \\ & \text { astango@bccn.uni-goettingen.de } \\ \text { Nationality } & \text { Italian }\end{aligned}$

\section{Work Experience}

October 2011 - September 2016

Researcher - Department of Neurorehabilitation Engineering, University Medical Center

Goettingen - Goettingen, Germany

- Implementation and simulation of antennas, channel modelling and SAR measurements for implant communications (IEEE 802.15.6) (3D electromagnetic simulations).

- Control of upper limb prostheses using spatial correlation of sEMG.

- Assisting editorial tasks for the books:" Introduction to neural engineering for motor rehabilitation" and "Surface Electromyography".

March 2007 - June 2011

Researcher - Aalborg University - Aalborg, Denmark

- Security requirements, application of enterprise security framework (SABSA) and risk analysis for critical information infrastructures.

- Access control methods for intrusion detection and prevention systems; security for IMS subsystems.

- Security requirements for Software Defined Radio (SDR) devices.

- Threat analysis for Federation of Personal Network.

July 2005 - February 2007

Researcher - Università' di Roma Tor Vergata - Rome, Italy

- Integration of air interfaces for high (IEEE 802.15.3) and low data rate (IEEE 802.15.4).

- Cross-layer optimization techniques in High Data Rate Wireless Personal Network.

May 2004 - June 2005

Researcher - Consorzio Università Industria - Laboratori di Radiocomunicazioni - RadioLabs Rome, Italy

- Analysis and simulations of media access control protocols for UWB systems. 


\section{Skills}

\section{Technical skills:}

- Good knowledge of 3D electromagnetic simulation (SEMCAD X and Sim4Life) and Networks simulation (ns-2)

- Good knowledge of Matlab and Simulink

- Knowledge of the most important wireless communication standards

- Basic knowledge of C++, AWK, OTCL, SQL

- Knowledge of SABSA (Sherwood Applied Business Security Architecture)

- $\quad$ Recording and processing EMG signals

- Competent with most Microsoft Office programs

\section{Personal skills:}

- Good ability to adapt to multicultural environments, gained working in UMG Goettingen, in Aalborg University and participating in European Projects.

- Autonomous and good in organizing the work.

\section{Education}

\section{September 2003}

M.Sc in Electronics Engineering

Università degli studi di L'Aquila, L'Aquila, Italy -Thesis title: "Study and simulation of the effect of the power control on ad hoc wireless local area network."

\section{Languages}

$\begin{array}{ll}\text { Italian } & \text { Mother tongue } \\ \text { English } & \text { Proficient user } \\ \text { German } & \text { Basic User }\end{array}$

\section{Additional information}

Paper reviewer in several conferences and journals. 